\title{
Maturation and nucleo-cytoplasmic shuttling of SnRNAs in Saccharomyces cerevisiae
}

\author{
Dissertation \\ for the award of the degree \\ "Doctor rerum naturalium" \\ of the Georg-August University Göttingen \\ within the doctoral program "Molecular Biology of Cells" \\ of the Georg-August University School of Science (GAUSS) \\ submitted by
}

Daniel Becker

from Cologne, Germany

Göttingen, February 2018 


\section{Members of the Thesis Committee}

Prof. Dr. Heike Krebber

Department of Molecular Genetics

Institute for Microbiology and Genetics

Prof. Dr. Ralf Ficner

Department of Molecular Structural Biology

Institute for Microbiology and Genetics

Prof. Dr. Ralph Kehlenbach

Department of Molecular Biology

Institute for Molecular Biology

\section{Members of the Examination Board}

Referee: Prof. Dr. Heike Krebber

Department of Molecular Genetics

Institute for Microbiology and Genetics

$2^{\text {nd }}$ Referee: Prof. Dr. Ralf Ficner

Department of Molecular Structural Biology

Institute for Microbiology and Genetics

\section{Further Members of the Examination Board}

Prof. Dr. Stefanie Pöggeler

Department of Genetics of Eukaryotic Microorganisms

Institute for Microbiology and Genetics

Prof. Dr. Jörg Stühlke

Department of General Microbiology

Institute for Microbiology and Genetics

PD Dr. Wilfried Kramer

Department of Molecular Genetics

Institute for Microbiology and Genetics

Date of oral examination: $24^{\text {th }}$ April 2018 


\section{Affidavit}

I hereby declare that this doctoral thesis entitled "Maturation and nucleo-cytoplasmic shuttling of snRNAs in Saccharomyces cerevisiae" has been written independently with no other sources and aids than quoted.

Göttingen, February 2018

Daniel Becker 


\section{Table of Contents}

Table of Contents

Table of Figures

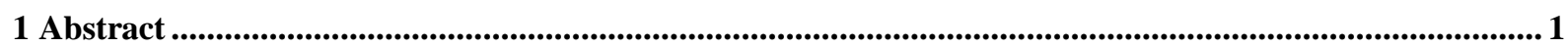

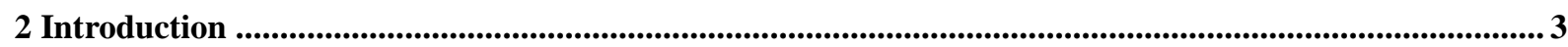

2.1 The lifecycle of Sm-class snRNAs in Saccharomyces cerevisiae ………..............................................

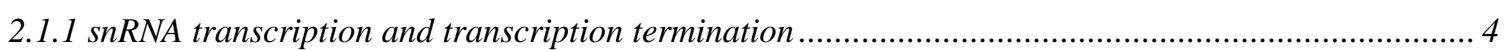

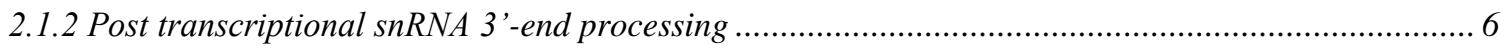

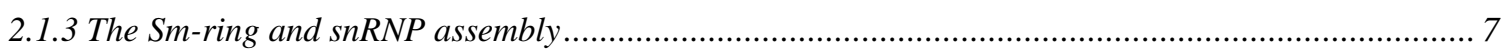

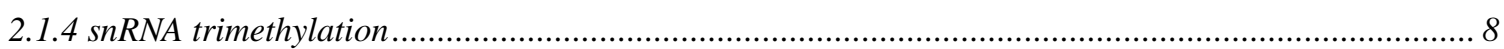

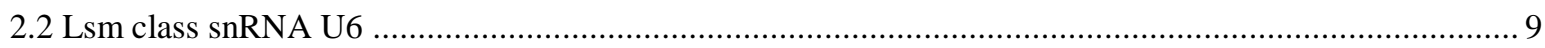

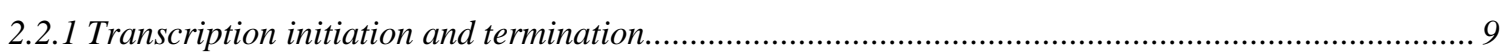

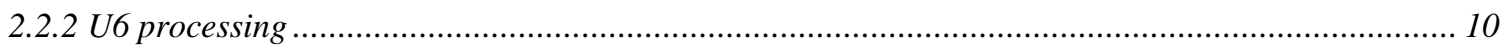

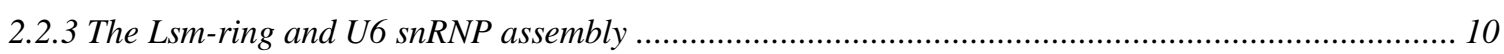

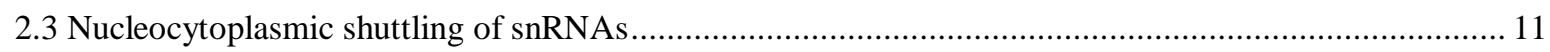

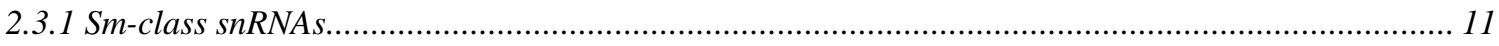

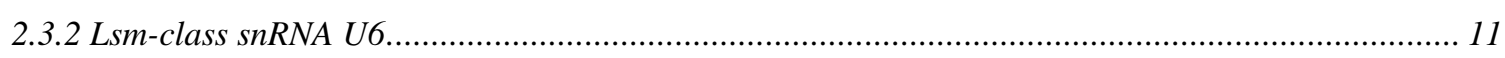

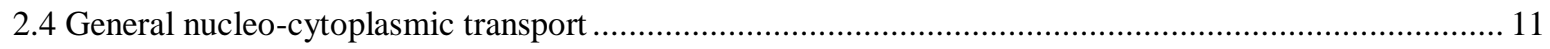

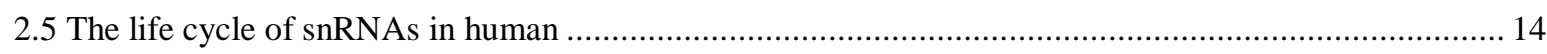

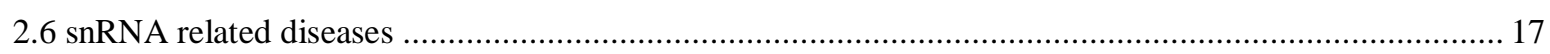

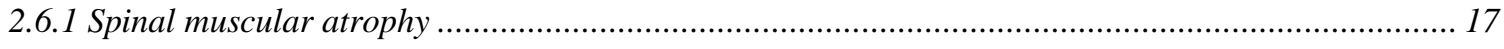

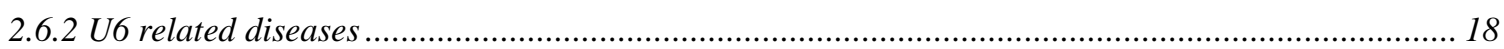

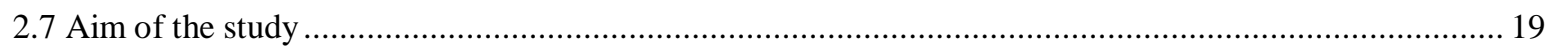

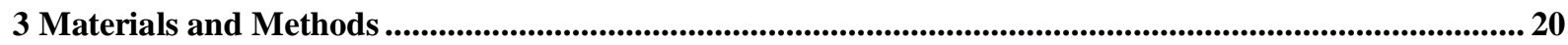

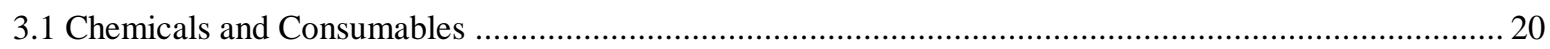

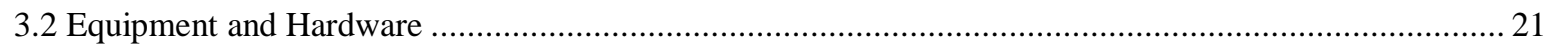

3.3 Software

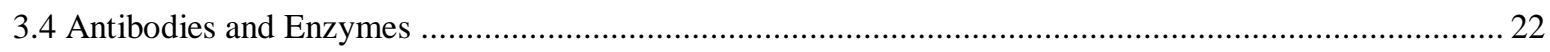

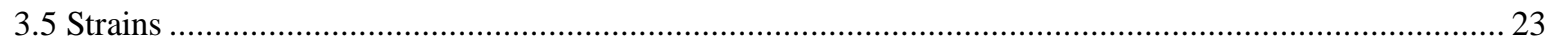

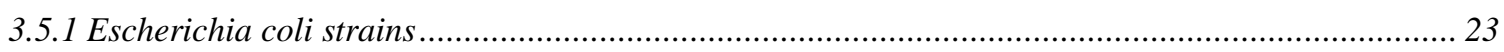

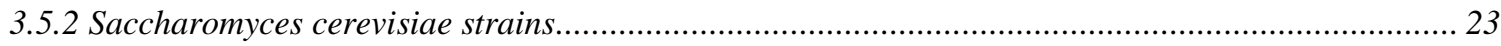

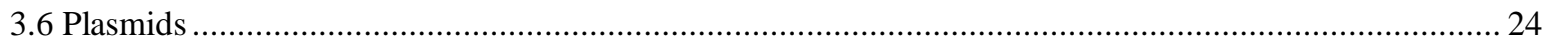

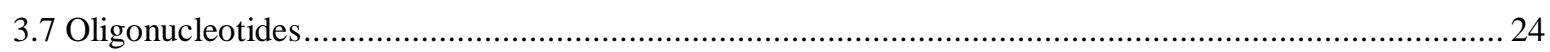

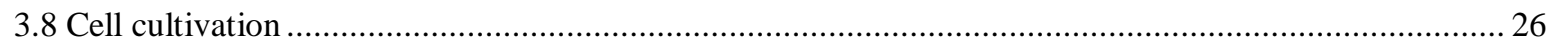

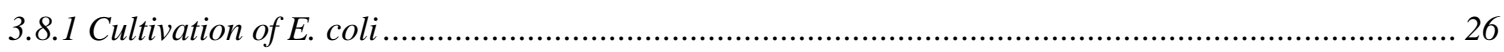

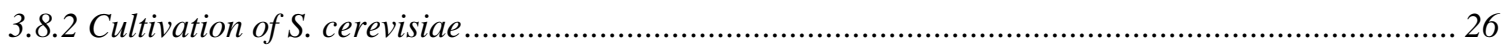

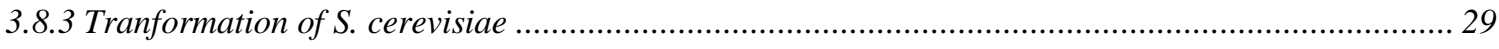

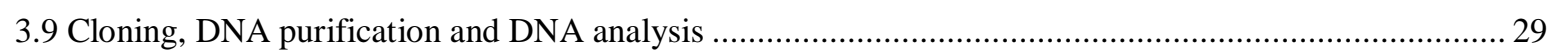




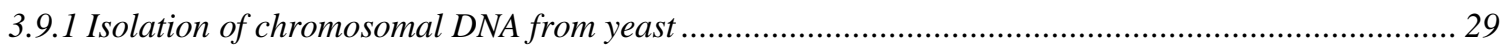

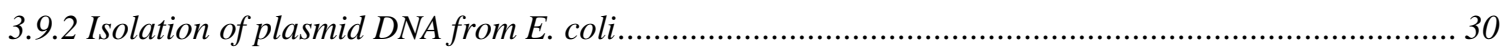

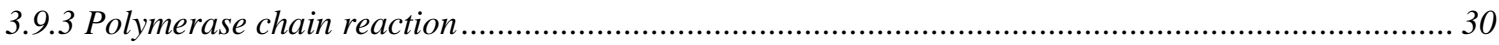

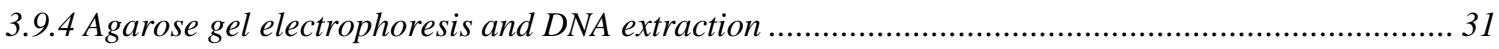

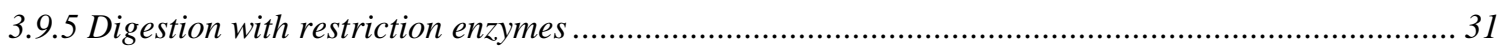

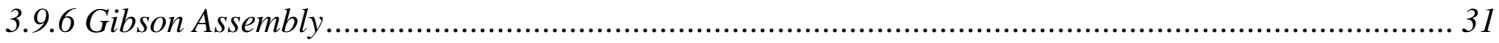

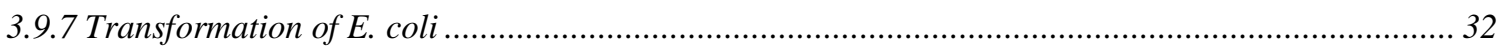

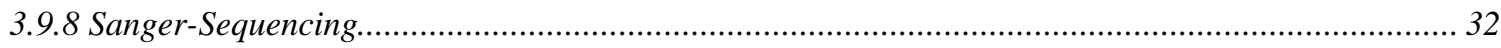

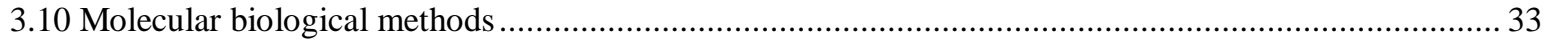

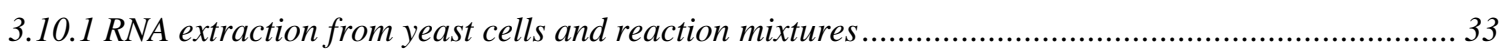

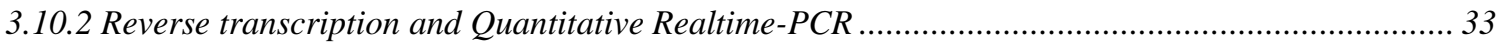

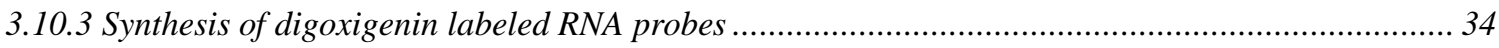

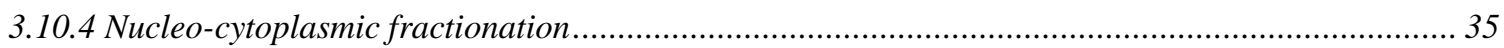

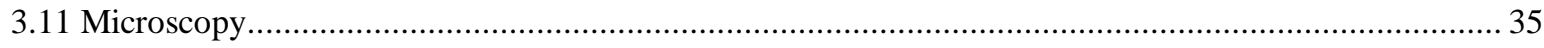

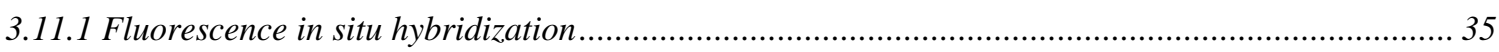

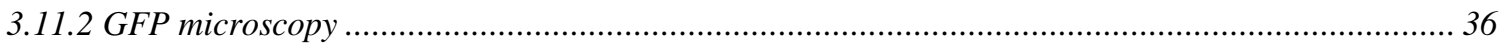

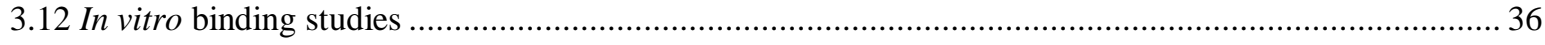

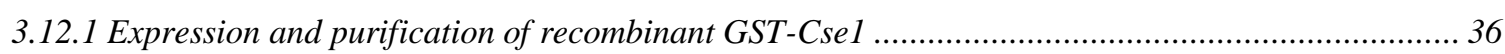

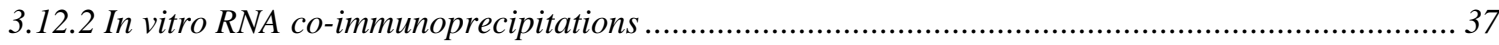

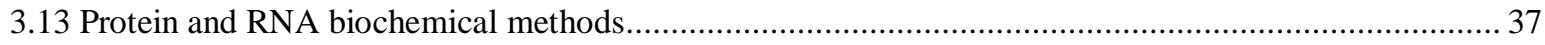

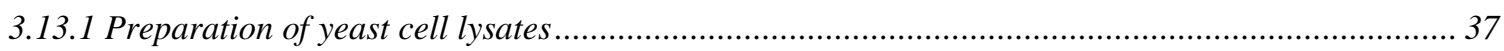

3.13.2 Protein-protein and protein-RNA co-immunoprecipitations ................................................. 38

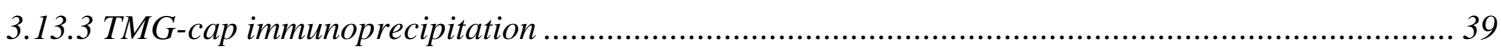

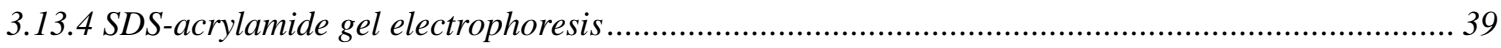

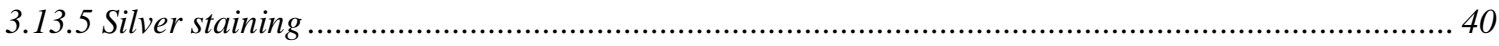

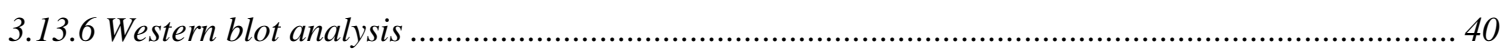

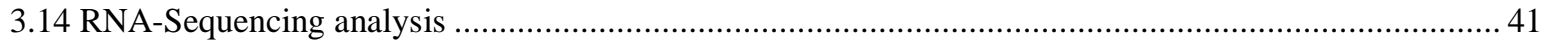

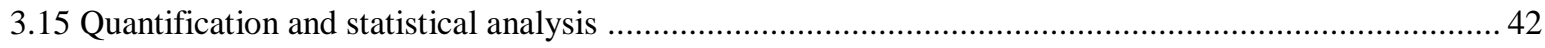

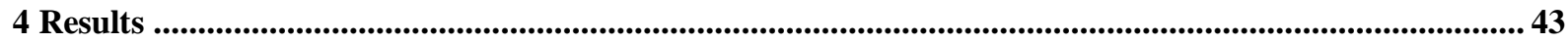

4.1 Yeast snRNAs are exported to the cytoplasm by Mex67 and Xpo1 …..........................................43

4.2 Mex67 and Xpo1 physically interact with the snRNAs in vivo and in vitro .........................................44

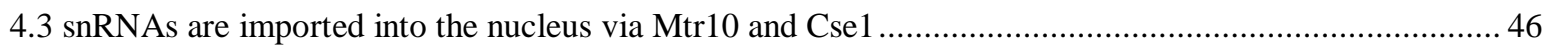

4.4 Sm-ring binding assembly takes place in the cytoplasm and is essential for snRNA re-import ..............49

$4.5 \mathrm{Cse} 1$ does not bind to snRNAs in vitro but contacts the Sm-ring via $\mathrm{SmB}$....................................51

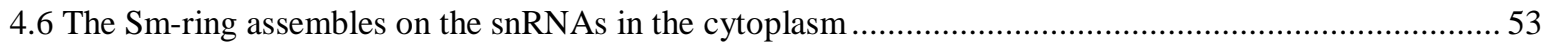

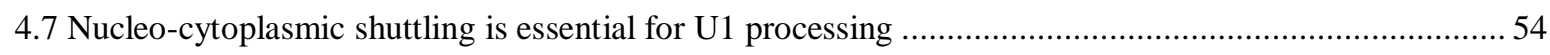

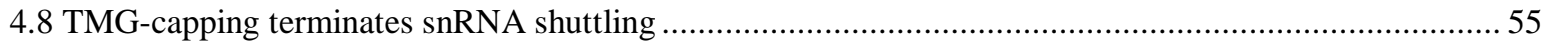

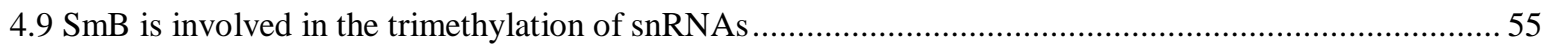

4.10 TMG-capped snRNAs preferentially interact with spliceosomal components and not with export factors 
4.11 Depletion of snRNAs from the nucleus results in splicing defects

4.12 Immature U1 is incorporated into the spliceosome and induces splicing defects.....

4.13 snRNA export block in mex67-5 xpo1-1 results in severe genome-wide splicing defects

5 Discussion

5.1 snRNAs require general mRNA export factors for nuclear export

5.2 Nuclear import of snRNAs is facilitated by Cse1 and Mtr10 in an Sm-ring-dependent manner

5.3 snRNP assembly occurs in the cytoplasm and is essential for subsequent snRNA import

5.43 '-processing of snRNAs takes place in the nucleus after nucleo-cytoplasmic shuttling and nucleolar TMG-capping finalizes snRNA processing by the inhibition of ongoing snRNP export

5.5 Nucleo-cytoplasmic shuttling is essential for correct spliceosome assembly and a fully functional splicing machinery

5.6 Nucleo-cytoplasmic shuttling represents an elegant quality assurance mechanism for snRNP assembly.. 72

5.7 Novel model of snRNP maturation in S. cerevisiae .73

6 References 


\section{Table of Figures}

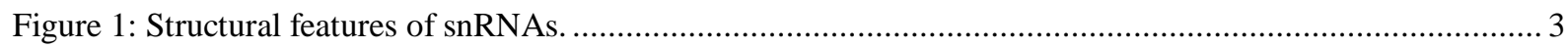

Figure 2: The CTD phosphorylation of $\mathrm{Ser}_{5}$ initiates the 5 ' -capping of Sm-class snRNAs..............................5

Figure 3: Termination of snRNA transcription is performed by the NNS-complex in yeast. ............................ 5

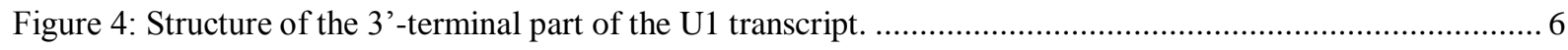

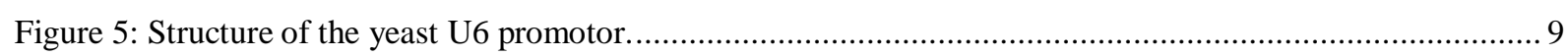

Figure 6: General Ran-dependent nuclear export and import. ........................................................... 12

Figure 7: Transcription initiation and transcription termination of Sm-class snRNAs in human..................... 14

Figure 8: RPAP2 and the Integrator complex terminate Sm-class snRNA transcription. ...............................15

Figure 9: Model for the nucleo-cytoplasmic shuttling of snRNAs in human cells. ...................................... 16

Figure 10: The Sm-ring assembles stepwise on the snRNAs following the so-called lid theory. ..................... 17

Figure 11: snRNAs show nuclear export defects in mRNA-export mutant strains......................................43

Figure 12: Mex67 and Xpo1 physically interact with the snRNAs in vivo.............................................. 45

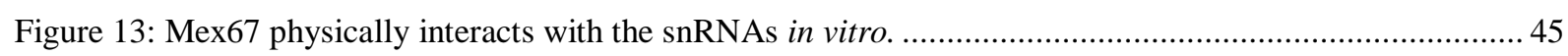

Figure 14: Analysis of import defects of snRNAs in mutants of all yeast karyopherins................................ 46

Figure 15: Cse1 and Mtr10 are responsible for the snRNA import................................................... 47

Figure 16: snRNAs are enriched in the cytoplasmic fractions of $c s e 1-1$ and $m t r 10 \Delta$ cells.............................48

Figure 17: Cse1 and Mtr10 physically interact with the snRNAs. ........................................................ 48

Figure 18: Cse1 and Mtr10 are essential for the correct Sm-ring localization..............................................49

Figure 19: The re-import of the Sm-ring is independent of the snRNAs.................................................50

Figure 20: The Sm-ring is essential for snRNA import into the nucleus.................................................50

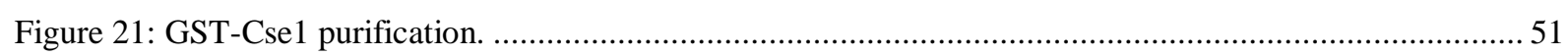

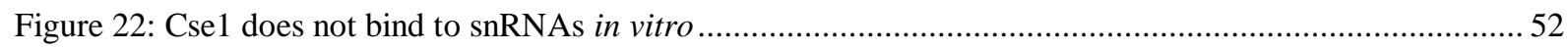

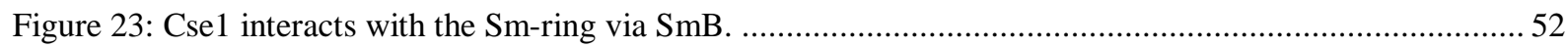

Figure 24: The Sm-ring assembles on the snRNAs after nuclear export in the cytoplasm. ...............................53

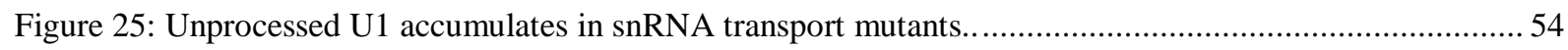

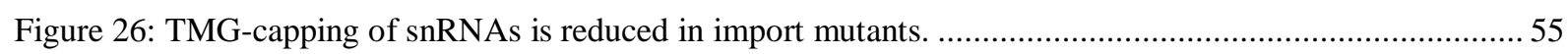

Figure 27: Depletion of SmB results in a nucleolar mislocalization of U1 and U2 ....................................56

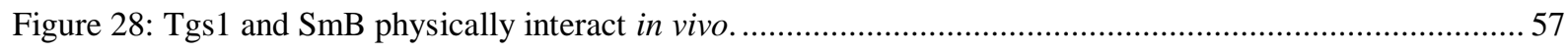

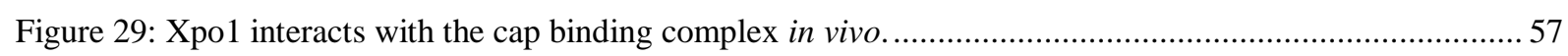

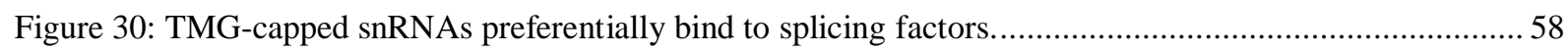

Figure 31: Depletion of snRNAs from the nucleus results in splicing defects. ...........................................59

Figure 32: An export block of snRNAs results in the incorporation of faulty processed U1 into the spliceosome.

Figure 33: Nearly one third of spliceosomes contain immature U1 snRNAs in mex67-5.............................61

Figure 34: Intron containing transcripts are enriched in spliceosomes that contain immature snRNAs.............61

Figure 35: snRNA export mutants show severe splicing defects. .....................................................6 62

Figure 36: RNA-Sequencing in mex67-5 xpo1-1 reveals genome wide splicing defects................................63

Figure 37: Detailed view of the RNA-Sequencing analysis of intron containing transcripts in mex67-5 xpo1-1. 64

Figure 38: Model for snRNA transport and snRNP maturation in S. cerevisiae.............................................. 74 


\section{Abstract}

While nucleo-cytoplasmic shuttling of snRNAs is a well known process in higher eukaryotes, it is still unknown whether snRNAs shuttle in Saccharomyces cerevisiae. Furthermore, it is unclear why snRNA shuttling evolved in general.

In yeast, snRNAs are transcribed in the nucleus and processed by nuclear endonuclease Rnt1 and exonuclease Rrp6. Additionally, snRNAs are trimethylated by the nucleolar methyltransferase Tgs1 before beeing integrated into spliceosomes. Therefore, a cytoplasmic phase seems unnecessary. However, in the present work we investigated a potential cytoplasmic phase of the snRNAs by fluorescent in situ hybridizations (FISH) and in vivo and in vitro RNA co-immunoprecipitation (RIP) experiments. We found that the snRNAs are exported from the nucleus by Mex67 and Xpo1 and determined the order of the subsequent maturation events. After export, the Sm-ring assembles on the snRNAs in the cytoplasm, which is an essential step for the nuclear import of the snRNP via the karyopherins Mtr10 and Cse1. In fact, FISH experiments revealed a cytoplasmic mislocalization of snRNAs after partial depletion of the Sm-ring, showing the importance of correct snRNP assembly. To answer the question if snRNAs are processed and TMG-capped before their export or after nucleo-cytoplasmic shuttling, we blocked snRNA shuttling. Interestingly, we detected increasing amounts of unprocessed U1 via qRT-PCR analysis in all transport mutants. In addition, we identified reduced amounts of TMG-capped snRNAs in import mutants, suggesting that snRNAs are processed and capped after shuttling and that they rather require the shuttling for subsequent processing steps. Furthermore, we performed a two step TMGRIP analysis with the spliceosomal proteins Prp40 and Prp17 as well as with Mex67 and Xpo1 and identified that TMG-capped snRNAs preferentially bind to the late splicing factors and not to Mex67 and Xpo1. Thus, we suggest a model in which trimethylation of snRNAs prevents further snRNA shuttling.

Importantly, we show that if shuttling is prevented, unprocessed snRNAs are incorporated into the spliceosomes, as the binding of Prp40 to immature U1 is significantly increased in mex67-5. Thus, spliceosomes cannot distinguish between mature and immature snRNAs and incorporate these immature snRNAs when they are not eliminated from the nuclear compartment. This in turn leads to severe genome wide splicing defects as confirmed by qRTPCR and RNA-Sequencing analysis. 
Thus, our data suggest that the export of pre-snRNAs is required to rapidly eliminate unprocessed snRNAs from the nucleus and thereby from the reach of spliceosomes to ensure a fully functional splicing machinery, which is essential for cell survival. 


\section{Introduction}

In eukaryotic cells, pre-mRNAs undergo several co- and posttranscriptional processing steps, such as 5'-capping, loading of proteins and finally the 3'-polyadenylation (Matera and Wang, 2014). An additional maturation step, which evolved in eukaryotic cells, is splicing. Eukaryotic genes contain non-coding regions, among them intron sequences, which allow higher eukaryotes to enrich their transcriptome by alternative splicing. The introns have to be removed properly before nuclear mRNA export occurs. This is essential to prohibit faulty transcripts from beeing translated by the cytoplasmic translation machinery, which could lead to toxic proteins. Splicing is performed by the spliceosome, which is composed of five spliceosomal subunits, which are highly homologue in all eukaryotes, named U1, U2, U4, U5 and U6 (Matera and Wang, 2014). Each subunit is a small nuclear ribonucleoprotein complex (snRNP) composed of one small nuclear (sn)RNA and several spliceosomal proteins. The snRNAs are a group of non-coding RNAs, which have an uridine-rich sequence (Matera et al., 2007). All snRNAs are essential for vegetative growth (Siliciano et al., 1991).

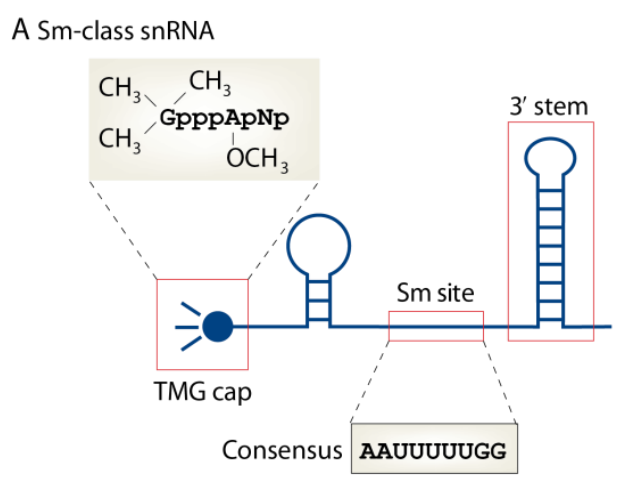

B Lsm-class snRNA

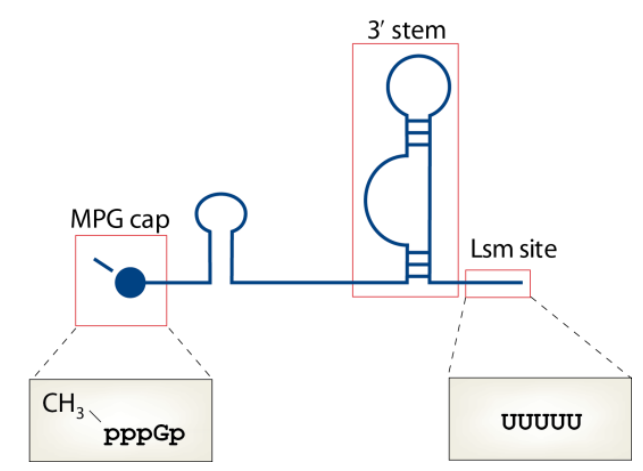

Figure 1: Structural features of snRNAs. snRNAs are divided into two classes, the Sm-class (U1, U2, U4 and U5) and the Lsm-class snRNA U6. (A) The Sm-class snRNAs contain a 5'-trimethylguanosine (TMG) cap, an Sm binding-site and a 3'-stem loop. (B) The Lsm-class snRNA U6 has a 5'-y-monomethyl (MPG) cap, an Lsm-binding site and a 3'-stem loop. Modified from (Matera et al., 2007)

The snRNAs can be differentiated into two classes: the Sm-class snRNAs U1, U2, U4 and U5 and the like Sm (Lsm)-class snRNA U6 (Figure 1). The Sm-class snRNAs are comprised of a 5'-trimethyl guanosine (TMG) cap, an Sm-binding site and a 3'-terminal stem loop (Matera et al., 2007). In contrast, the Lsm snRNA U6 has a 5' $-\gamma$-monomethyl cap, an Lsm-binding site and also a 3'-terminal stem loop (Matera et al., 2007; Reddy et al., 1987; Singh and Reddy, 1989). 


\subsection{The lifecycle of Sm-class snRNAs in Saccharomyces cerevisiae}

\subsection{1 snRNA transcription and transcription termination}

Similar to mRNAs, the Sm-class snRNAs are transcribed by the RNA polymerase II (RNAP II). In contrast, the Lsm-class snRNA U6 is transcribed by RNA polymerase III (RNAP III) (Cramer et al., 2008; Kunkel et al., 1986; Will and Lührmann, 2001).

Transcription initiation depends on several transcription factors (TFIIA, TFIIB, TFIID, TFIIE, TFIIF and TFIIH), the Mediator complex and the RNAP II with an unphosphorylated Cterminal domain (CTD). Expression regulation is mediated by the different binding patterns of these factors to the genes' promoter (Björklund and Gustafsson, 2005; Hsin and Manley, 2012).

RNAP II comprises 12 subunits, which build the structural core enzyme (Cramer et al., 2008). The catalytic active subunit of RNAP II is Rpb1, which contains a CTD enhancing or modulating several processing steps of the transcript (Hsin and Manley, 2012). One important step in the processing of RNAP II transcripts is the addition of a 5'-monomethyl cap. Directly after the start of transcription, the newly synthesized transcript obtains its $5^{\prime}$ - $\mathrm{m}^{7} \mathrm{G}$-cap. In vitro experiments indicated that capping occurs already after 20 nucleotides have been transcribed (Coppola et al., 1983; McCracken et al., 1997). In yeast, the CTD consists of 26 heptad repeats of the amino acids $\mathrm{Tyr}_{1} \mathrm{Ser}_{2} \mathrm{Pro}_{3} \mathrm{Thr}_{4} \mathrm{Ser}_{5} \mathrm{Pro}_{6} \mathrm{Ser}_{7}$. This domain is highly conserved throughout eukaryotes and only the number of heptad repeats differs between species, e.g. 52 in human (Hsin and Manley, 2012). During the transcription process, the amino acids $\mathrm{Ser}_{2}$ and $\mathrm{Ser}_{5}$ of the CTD are modified by phosphorylation and dephosphorylation. $\mathrm{Ser}_{2}$ is mainly phosphorylated at the end of transcription, whereas Ser $_{5}$-phosphorylation is present at the beginning of transcription and a prerequisite for 5'-capping as it supports recruitment of the guanylyltransferase Ceg1 to the nascent transcript (Figure 2) (Cho et al., 1997). Ceg1 and the RNA triphosphatase Cet1 perform the 5'-capping by a three-step process. This 5'-capping is essential for RNA stability (Jiao et al., 2010; Schwer et al., 1998). First, Cet1 hydrolyzes a phosphate of the 5'-triphosphate, followed by the addition of a guanosine monophosphate (GMP) to the diphosphate by Ceg1. Finally, the methyltransferase Abd1 adds a methylgroup to the $\mathrm{N}_{7}$ group of the guanine base (Gu et al., 2010). 


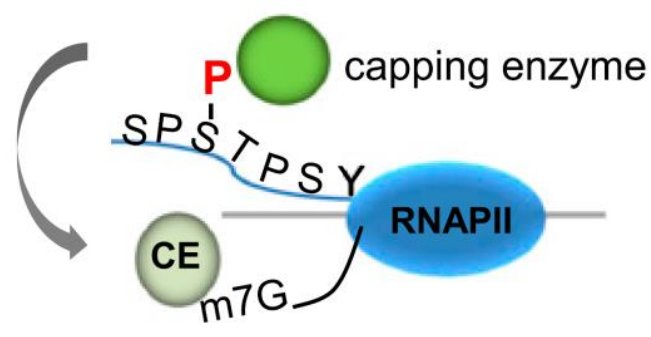

Figure 2: The CTD phosphorylation of $\mathrm{Ser}_{5}$ initiates the 5'-capping of Sm-class snRNAs. The phosphorylation of Ser ${ }_{5}$ leads to the recruitment of the capping enzymes (CE) Ceg1 and Cet1 to the nascent transcript. Both Ceg1 and Cet1 perform the addition of the $5^{\prime}-\mathrm{m}^{7} \mathrm{G}$-cap by hydrolyzing the 5' triphosphate and adding a GMP to the diphosphate. Taken from (Hsin and Manley, 2012)

Upon capping, first, the $\mathrm{m}^{7} \mathrm{G}$-cap of mRNAs is recognized by the cap binding complex (CBC) consisting of Cbp20 and Cbp80 (Lewis and Izaurralde, 1997; Schwer et al., 2011). Second, there is strong evidence for the co-transcriptional loading of the serine-arginine (SR)-rich RNA binding protein Npl3 to the nascent mRNA transcript during mRNA transcription. Npl3 interacts with the RNAP II at the site of transcription initiation (Lei et al., 2001) and with the CBC (Baejen et al., 2014; Shen et al., 2000). Npl3 also interacts with the mature U1 snRNP (Gottschalk et al., 1998), but up to date it is unclear whether Npl3 is also loaded onto the snRNAs co-transcriptionally or at a later time point.

Npl3 is needed for correct transcription termination of several non-coding RNAs, e.g. small nucleolar (sno)RNAs but not for snRNAs. SnoRNAs appear as longer transcripts in an $n p l 3 \Delta$ strain, in contrast to snRNAs, which show no elongated transcripts in $n p l 3 \Delta$ (Holmes et al., 2015). The snRNA transcription termination process is performed by the NNS (№rd1-Nab3-

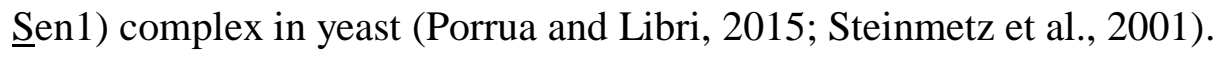

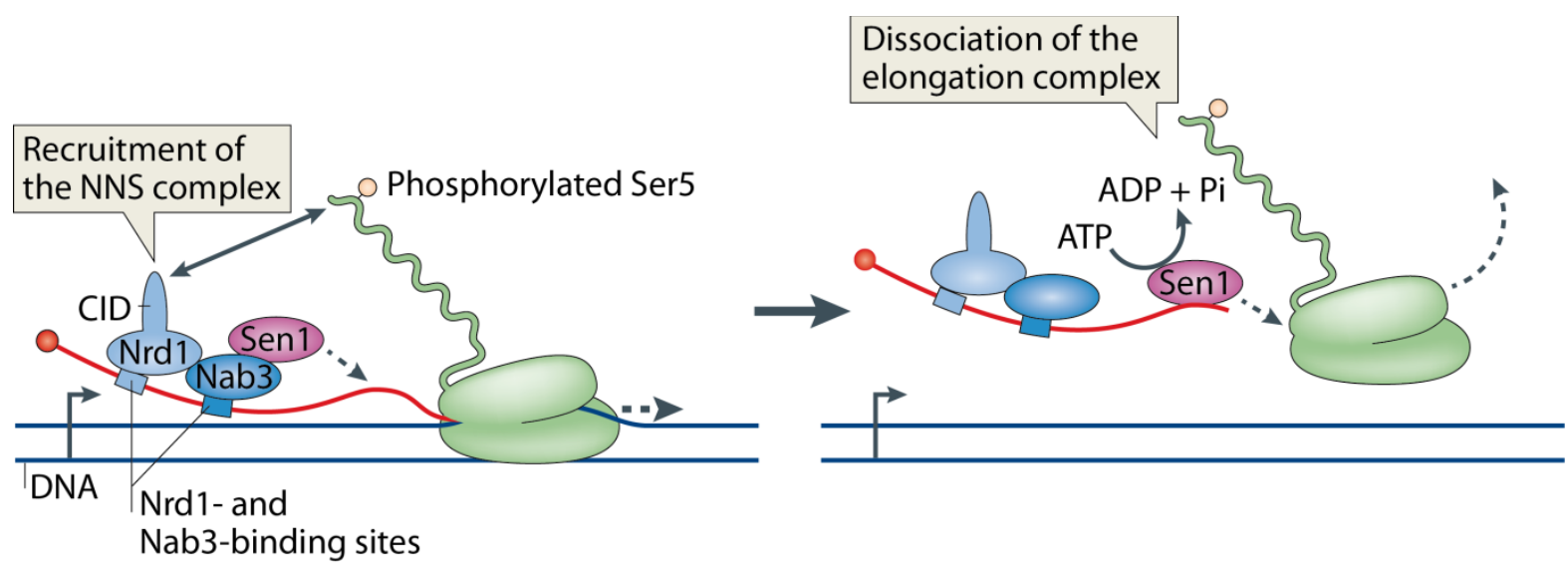

Figure 3: Termination of snRNA transcription is performed by the NNS-complex in yeast. Nrd1 and Nab3 are recruited to the RNAP II. The loading onto the RNAP II is mediated by the interaction of the CTD-interacting domain (CID) of Nrd1 with the phosphorylated $\mathrm{Ser}_{5}$ of the RNAP II CTD. The loading of Nrd1 and Nab3 onto the nascent transcript, allows both Nrd1 and Nab3 to recognize snRNA-specific termination signals on the transcript. Finally, Sen1 terminates the transcription. Modified from (Porrua and Libri, 2015) 
Here again, the modification status of the CTD plays a crucial role. Nrd1 is recruited to the RNAP II early after transcription initiation as Ser $_{5}$ phosphorylation of the CTD favors Nrd1 binding to RNAP II (Figure 3) (Heo et al., 2013; Kubicek et al., 2012). Nrd1 and Nab3 bind as a heterodimer to RNAP II via its CTD-interacting domain (CID) (Carroll et al., 2007). The early loading onto RNAP II allows Nrd1 and Nab3 to recognize snRNA specific termination signals (GUAA/G and UCUUG, respectively) on the transcript (Porrua et al., 2012). After recognition of the termination signal, Nrd1 and Nab3 recruit the RNA helicase Sen1. Sen1 terminates transcription by a process, which is still not completely understood (Creamer et al., 2011; Ghaemmaghami et al., 2003; Porrua and Libri, 2015).

\subsubsection{Post transcriptional snRNA 3'-end processing}

After transcription termination, the final snRNA transcript needs to be processed at its 3'-end. For snRNAs, this final processing step is not facilitated by factors that were already cotranscriptionally loaded. While the exact mechanism of snRNA 3'-processing in higher eukaryotes is still unclear (Matera and Wang, 2014), the nuclear endoribonuclease Rnt1 has been identified to perform 3'-processing of snRNAs in yeast (Catala et al., 2004; Seipelt et al., 1999). Depletion of Rnt1 leads to the accumulation of enlarged pre-cursors of the snRNAs U1, U2, U4 and U5 (Allmang et al., 1999; Chanfreau et al., 1997; Elela and Ares, 1998; Seipelt et al., 1999). U1, which is encoded by SNR19, is transcribed as a 643 nucleotide long pre-cursor, which is processed to the 75 nucleotide shorter mature form (568 nucleotides in length). The shorter form is predominantly found in cells (Seipelt et al., 1999; Shukla and Parker, 2014). Within this 3'-extension, two consensus sequences for the endoribonuclease Rnt1 have been identified (Figure 4) (Seipelt et al., 1999).

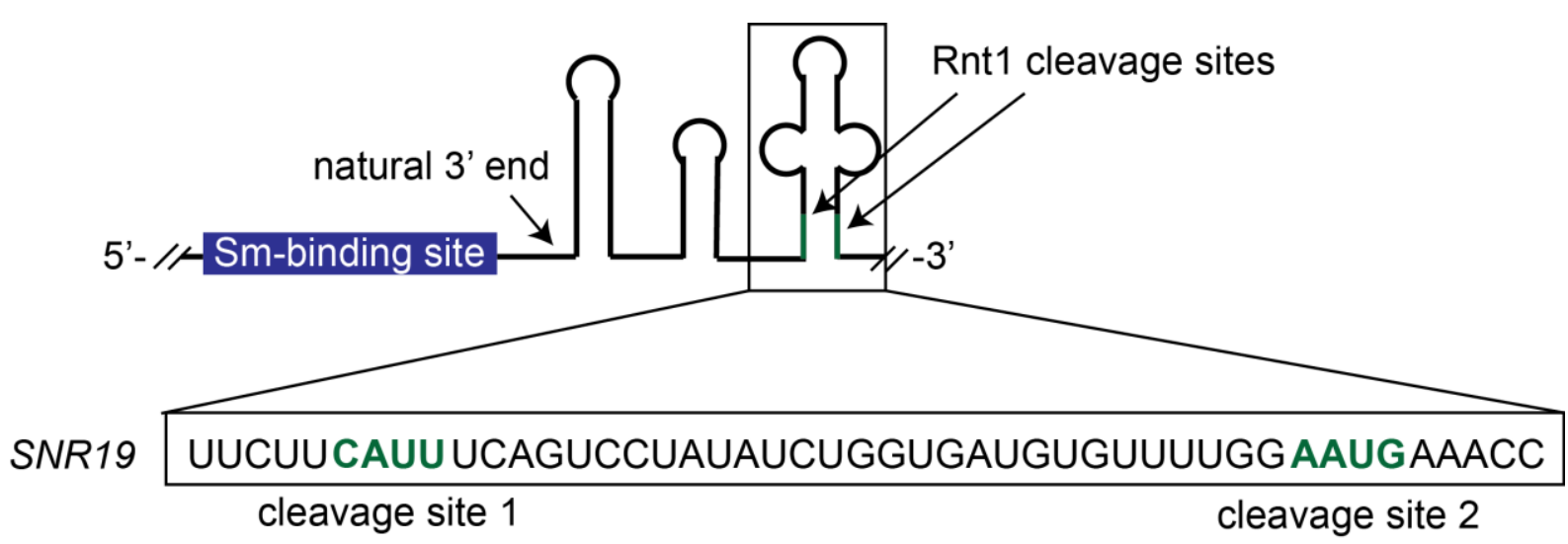

Figure 4: Structure of the 3'-terminal part of the U1 transcript. The endoribonuclease Rnt1 cleaves the U1 transcript at two distinct sites downstream of the natural 3'-end of U1. Disruption of the cleavage site or depletion of Rnt1 from cells leads to a 75 nucleotide longer U1. 
After the Rnt1 mediated cleavage, the remaining nucleotides of the 3'-overhang are degraded by the nuclear exosome to receive the mature U1 transcript (Allmang et al., 1999; Coy et al., 2013). Mutants of the yeast exosome show an accumulation of poly-adenylated forms of U1 and U4 (Coy et al., 2013; van Hoof et al., 2000). If the nuclear exonuclease Rrp6 is depleted from the cells, snRNAs are polyadenylated by the poly(A) polymerase Pap1 after Rnt1 mediated cleavage (van Hoof et al., 2000). These poly-adenylated snRNAs finally result in splicing defects (Coy et al., 2013).

The exosomal degradation of snRNAs stops at the Sm-ring. U1 contains an Sm-ring binding site composed of nine nucleotides upstream of its mature 3'-end, at nucleotide position 553559 (AUUUUUGA) (Seipelt et al., 1999). Deletion of the Sm-binding site leads to the 3'- to 5'-degradation of the snRNA by the nuclear exosome and the unhindered 5'- to 3'degradation by the decapping enzyme Dcp2 accompanied by the 5'- to 3'-degrading exoribonuclease Xrn1 (Coy et al., 2013; Seipelt et al., 1999; Shukla and Parker, 2014). In fact, the insertion of the Sm-ring binding site into the cryptic unstable transcript (CUT) NTS1 leads to its stabilization. Thus, the Sm ring functions as a stop signal for nuclear exosome-mediated degradation (Coy et al., 2013).

\subsubsection{The Sm-ring and snRNP assembly}

In S. cerevisiae, the Sm-ring consists of a seven membered ring of the Sm-proteins SmB, SmD1, SmD2, SmD3, SmE, SmF and SmG, which are encoded by the genes SMB1, SMD1, SMD2, SMD3, SME1, SMX2 and SMX3, respectively (Matera and Wang, 2014; Pettersson et al., 1984). All proteins are highly conserved in all eukaryotes and essential for vegetative growth. Each Sm-protein comprises an Sm domain, which consists of two regions (Sm1 and Sm2) with a linker in between (Hermann et al., 1995; Séraphin, 1995). The hydrophobic amino acids within this Sm domain are essential for the protein-protein interactions of Smproteins (Camasses et al., 1998). The ring is pre-formed in three sub-complexes, in detail, SmB-SmD3, SmF-SmE-SmG and SmD1-SmD2 (Kambach et al., 1999). Each of the seven Sm-ring members has an RNA binding site. Therefore, the Sm-ring assembles around the snRNA, through both the interaction between neighboring Sm proteins and the proteinsnRNA interactions ( $\mathrm{Li}$ et al., 2016). How the assembly process is accomplished in detail is still unknown in yeast. Brr1, the yeast homologue of human Gemin2, is essential for the yeast Sm-ring assembly similar to Gemin2 for the formation of the human Sm-ring (Kroiss et al., 2008; Liu et al., 1997; Noble and Guthrie, 1996; Zhang et al., 2011). A recent study revealed the structural similarity between Brr1 and Gemin2 (Schwer et al., 2017). The C-terminal 
hexapeptide of Brr1, including the two conserved amino acids Gln336 and Glu338 and the Nterminal amino acids 24-47, are essential for the interaction with the Sm-proteins. Brr1 supports the assembly of SmD1, SmD2, SmE and SmF. Brr1 is not essential in yeast, but brr1 4 shows synthetic lethality with Sm-protein mutants, which have single amino acid exchanges in their protein binding sites. Hence, Brr1 may be a first evolutionary step of the SMN-Gemin2-complex in higher eukaryotes (Schwer et al., 2017).

Five of the seven Sm-ring members ( $\mathrm{SmB}, \mathrm{SmD}$, $\mathrm{SmE}, \mathrm{SmF}$ and $\mathrm{SmG}$ ) were part of a screening experiment to identify their individual RNA binding sites. The loss of one RNA binding site, except the SmF RNA-binding site, did not affect cell growth. However, the mutation of single amino acids in the RNA binding sites of two Sm-proteins is lethal for the cells, showing that only one incorrect binding site can be compensated by the others (Schwer and Shuman, 2015; Schwer et al., 2016).

The mechanism of the import of the Sm-proteins into the nucleus is unknown. SmB, SmD1 and SmD3 contain nuclear localization signal (NLS)-like domains at their C-terminal ends after the Sm motif, which are not conserved in humans. The NLSs contain lysine- and arginine-rich regions, similar to classical NLSs, e.g. the SV40-NLS (Bordonné, 2000; Dingwall and Laskey, 1991). Both NLS-sequences have nuclear localization properties as it has been shown by GFP-localization experiments (Bordonné, 2000). Interestingly, the SV40NLS could not replace the SmB-NLS. Therefore, it was hypothesized that the Sm-proteins are imported via other specific importins and probably not by the classical importin $\alpha$ and importin $\beta$ pathway. Furthermore, it was clearly shown, that the deletion of one NLS is not enough to deplete the Sm-ring from the nucleus. Simultaneous deletion of the SmB- and SmD1-NLS, however, results in the loss of the nuclear Sm-ring localization and finally in cell lethality (Bordonné, 2000).

\subsection{4 snRNA trimethylation}

An additional processing step in the snRNA lifecycle is the hypermethylation of the $\mathrm{m}^{7} \mathrm{G}$-cap at the 5'-end. All four RNAP II snRNA transcripts (U1, U2, U4 and U5) receive a 5' -2,2,7trimethyl guanosine (TMG) cap (Figure 1) (Reddy et al., 1992). This step is performed by the trimethylguanosine synthase I (Tgs1, encoded by TGS1), which is located in the nucleolus in yeast (Mouaikel et al., 2002). Deletion of TGS1 results in a cold sensitive growth defect and leads to severe splicing defects and ribosomal (r)RNA processing defects (Colau et al., 2004; Mouaikel et al., 2002). Tgs1 builds a functional homodimer via its N-terminal domain. This homodimerization is essential for Tgs1 functionality and its nucleolar localization (Boon et 
al., 2015). In addition, Tgs1 affects the snRNP composition. In cells lacking TGS1 (tgs14), U1 snRNPs accumulate with high amounts of the yeast CBC proteins Cbp20 (CBP2) and Cbp80 (STO1). This observation indicates that the CBC can in principle bind to the $\mathrm{m}^{7} \mathrm{G}$-cap of yeast snRNAs. Furthermore, the deletion of the $\mathrm{m}^{7} \mathrm{G}$-cap binding capability of Cbp20 rescues a cold sensitive growth defect of $\operatorname{tgs} 1 \Delta$ cells. Interestingly, the increased binding of the CBC to the U1 snRNP does not alter the snRNP composition itself (Schwer et al., 2011). As the Sm-ring is essential for the snRNA stability, it seems possible that the Sm-ring is recruited quite early to the snRNA to protect it from exonucleolytic digestion (Coy et al., 2013; Seipelt et al., 1999; Shukla and Parker, 2014). Hence, the TMG-capping could be a subsequent event in the snRNA lifecycle, in particular because SmB is involved in snRNA trimethylation as it interacts with Tgs1 in vitro (Bordonné, 2000; Mouaikel et al., 2002; Plessel et al., 1994).

\subsection{Lsm class SnRNA U6}

\subsubsection{Transcription initiation and termination}

The Lsm-class snRNA U6 is encoded by the SNR6 gene, which is transcribed by RNAP III (Schramm and Hernandez, 2002; Turowski and Tollervey, 2016). The U6 promotor consists of a 5' upstream TATA-box, an intergenic A-box and a 3' downstream B-box, which are all essential for U6 transcription initiation (Figure 5). The A- and B-box elements are characteristic for RNAP III transcripts and are essential for the binding of TFIIIC (Schramm and Hernandez, 2002; Turowski and Tollervey, 2016). Within SNR6, the B-box is located 120 base pairs downstream of the coding region (Brow and Guthrie, 1990).

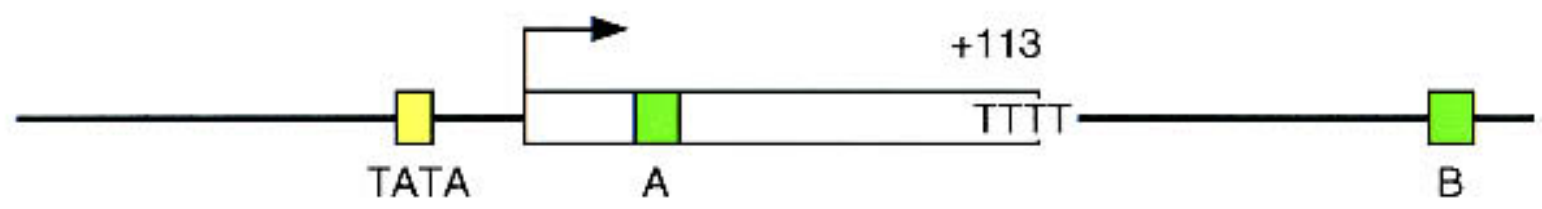

Figure 5: Structure of the yeast U6 promotor. The yeast U6 promotor consists of a TATA-box, an A-site and a downstream B-box. All three features are essential for correct U6 transcription. Taken from (Schramm and Hernandez, 2002)

The TATA-box of SNR6 is located at nucleotide position -31 to -29 and is highly conserved (Brow and Guthrie, 1990). It has been shown that one protein of the TFIIIB-complex, namely the TATA-binding protein (TBP or Spt15 in S. cerevisiae), is not only essential for the recognition of RNAP II substrates, but is also responsible for RNAP III and RNAP I transcribed genes in both yeast and human (Lobo et al., 1992; Margottin et al., 1991). In addition, the TATA-box is essential for the correct recognition of the transcription start site 
(TSS). Deletions of either the TATA-box or the A-site result in downstream or upstream shifts of the SNR6 TSS, respectively (Eschenlauer et al., 1993).

SNR6 transcription termination occurs at downstream oligo(dA) stretches. These stretches differ between $\mathrm{A}_{4}$ (human) and $\mathrm{A}_{5}$ to $\mathrm{A}_{6}$ in yeast. The weak interaction between the oligo(dA) of the template strand and the oligo(U) of the nascent RNA acts as a signal for transcription termination (Turowski and Tollervey, 2016). This oligo(U) stretch of the U6 transcript also acts as the Lsm binding site (Matera et al., 2007).

\subsubsection{U6 processing}

The oligo(U) stretch of U6 needs to be processed for U6 stability (Mroczek et al., 2012). This is performed by the putative phosphodiesterase Usb1 (USB1), which is located in the nucleus. Usb1 acts as an exonuclease, trims the oligo(U)-stretch and finally generates a 3'- phosphate for U6 stabilization. The depletion of USB1 results in splicing and cell growth defects, which can be suppressed by additional SNR6 copies (Mroczek et al., 2012).

U6 is differently capped than the other Sm-class snRNAs. U6 does not receive an $\mathrm{m}^{7} \mathrm{G}$-cap and is not trimethylated. However, it gets a cap-structure, which is a $5^{\prime}-\gamma$-monomethyl-cap in yeast as well as in human cells (Reddy et al., 1987; Singh and Reddy, 1989). While in human capping is performed by the BCDIN3 methyltransferase, the yeast capping enzyme still needs to be identified (Jeronimo et al., 2007; Shimba and Reddy, 1994).

\subsubsection{The Lsm-ring and U6 snRNP assembly}

An Lsm-ring consists of seven Lsm-proteins. The Lsm1-7 and the Lsm2-8 are the best characterized Lsm-rings. Lsm1-7 is located in the cytoplasm, where it is involved in mRNA degradation, whereas Lsm2-8 (Lsm8-Lsm2-Lsm3-Lsm6-Lsm5-Lsm7-Lsm4) is localized in the nucleus and binds to U6 (Wilusz and Wilusz, 2013). In contrast to the Sm-ring, which needs RNA to assemble ( $\mathrm{Li}$ et al., 2016), the Lsm-ring can build its doughnut-shaped form in the absence of RNA. It specifically binds to the oligo(U) stretch of U6 and cannot bind the canonical Sm-binding site of Sm-class snRNAs (Achsel et al., 1999). After the association of the Lsm-ring with U6, the binding of the Lsm-ring promotes the assembly of the U4/U6 disnRNP (Achsel et al., 1999; Hardin et al., 2015). The deletion of Lsm-ring members causes a cytoplasmic mislocalization of U6 (Spiller et al., 2007). 


\subsection{Nucleocytoplasmic shuttling of snRNAs}

\subsubsection{Sm-class snRNAs}

In contrast to human cells, the 3'-end processing and the 5'-trimethylation of yeast Sm-class snRNAs takes place in the nucleus (Catala et al., 2004; Mouaikel et al., 2002). Therefore, yeast snRNAs are expected to stay in the nucleus (Matera and Wang, 2014; Sloan et al., 2016; Vasianovich and Wellinger, 2017; Will and Lührmann, 2001). Interestingly, it was demonstrated in a heterokaryon assay that snRNAs can shuttle into the cytoplasm, but it was assumed to be an artifact (Olson and Siliciano, 2003). In addition, in previous studies we could show that also other non-coding RNAs, which act in the nucleus, like the telomerase RNA TLC1, shuttle into the cytoplasm for RNP assembly (Wu et al., 2014). Although many models suggest that snRNAs do not shuttle in yeast, this has not been investigated systematically so far (Matera and Wang, 2014; Sloan et al., 2016; Vasianovich and Wellinger, 2017; Will and Lührmann, 2001).

\subsubsection{Lsm-class SnRNA U6}

The Lsm-class snRNA U6 is mostly proposed to remain nuclear in all eukaryotes (Matera and Wang, 2014). Nevertheless, there are publications that describe cytoplasmic U6. First, a heterokaryon could show shuttling of U6, but it was assumed to be an artifact (Olson and Siliciano, 2003). Second, the Lsm-ring was shown to be essential for U6 nuclear retention (Spiller et al., 2007). Therefore, to date it cannot be ruled out if U6 shuttles into the cytoplasm.

\subsection{General nucleo-cytoplasmic transport}

Cellular compartmentalization is a key feature of eukaryotic cells. It allows cells to separate different cellular processes from each other. Transport between nucleus and cytoplasm occurs across the nuclear envelope through nuclear pore complexes (NPC).

There are two kinds of export pathways in yeast cells. On the one hand, the Ran-dependent transport by karyopherins and on the other hand, the export of RNAs via Mex67.

Ran (or Gsp1 in yeast) is a small protein, which is present in the cell in two states: The GTPand the GDP-bound state. Ran-GTP is more abundant in the nucleus, whereas Ran-GDP is mostly present in the cytoplasm. In yeast, this asymmetric cellular distribution over the nuclear envelope is maintained by nuclear Prp20 (RCC1 in human) and cytoplasmic Rna1 (Ran-GAP in human) (Aitchison and Rout, 2012; Sloan et al., 2016). Prp20, the yeast Ran guanine nucleotide exchange factor (Ran-GEF), is located in the nucleus and is essential for 
the maintenance of the Ran-GTP-Ran-GDP gradient (Akhtar et al., 2001; Fleischmann et al., 1991; Sloan et al., 2016). In contrast, the Ran-GTPase activating protein (Ran-GAP or Rna1) is located in the cytoplasm and stimulates the hydrolysis of Ran-GTP to Ran-GDP (Hopper et al., 1990; Sloan et al., 2016). Taken together, the Ran-gradient allows the directed transport of cargoes out of the nucleus into the cytoplasm and vice versa.
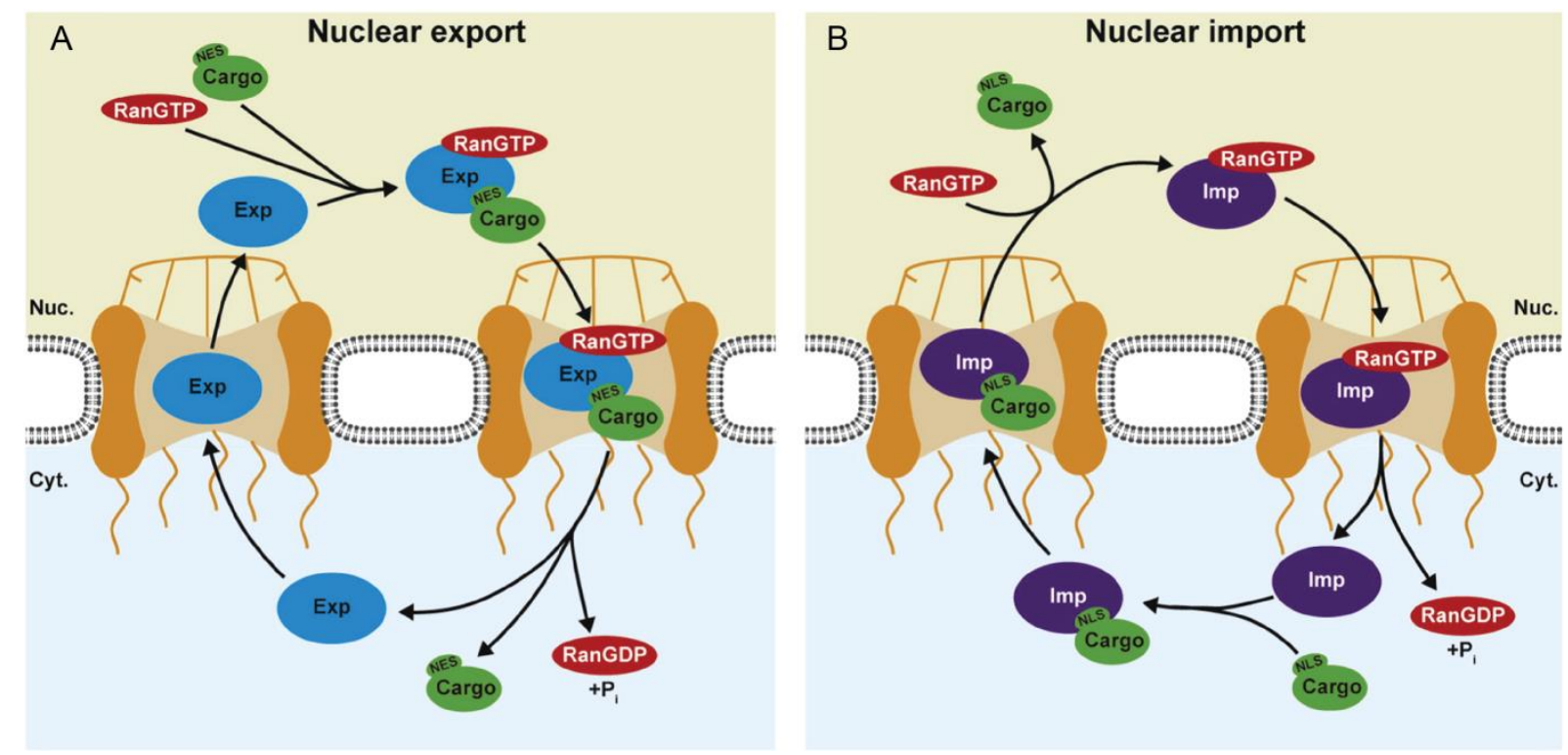

Figure 6: General Ran-dependent nuclear export and import. (A) The nuclear export of cargoes from the nucleus into the cytoplasm is initiated by the binding of an exportin along with Ran-GTP to an export competent substrate, e.g. an NES containing protein. Once the export complex is translocated through the hydrophobic meshwork of the NPC into the cytoplasm, the export cargo is released by Ran-GTP hydrolysis via Rna1 in yeast or Ran-GAP in human, respectively. (B) Nuclear import complexes can form freely in the cytoplasm and move through the NPC into the nucleus. There, the import complex is released by the loading of Ran-GTP via Prp20 in yeast or RCC1 in human, respectively. Modified from (Sloan et al., 2016)

The transport itself is facilitated by specific proteins, the karyopherins. In yeast, there are 14 karyopherins functioning as exportins or importins (Fried and Kutay, 2003; Hahn and Schlenstedt, 2011). In the nucleus, the exportin binds to its substrate (Figure 6) (Aitchison and Rout, 2012). This binding is promoted by the simultaneous binding of Ran-GTP. Afterwards, the specific structure of the exportin allows the transport of the whole cargo-complex through the NPC. The NPC is a large protein complex, which connects the nucleus and the cytoplasm by interrupting the nuclear membrane. It consists of nuclear porins (called Nups), which are assembled as an outer and an inner layer. The inner layer is highly hydrophobic to prevent diffusion. The hydrophobicity is obtained by Nups with several phenylalanine-glycine (FG)rich repeats. Rna1 is bound to proteins on the cytoplasmic site (Aitchison and Rout, 2012). Here, the cargo is released by GTP-hydrolysis. The exportin recycles back to the nucleus and the Ran-GDP is re-imported by Ntf2 (Ribbeck et al., 1998; Sloan et al., 2016). The import 
only requires the assembly of the importin and the cargo. In the nucleus, the cargo is released through binding of Ran-GTP, which was charged by Prp20 (Sloan et al., 2016).

The exportin Xpo1 (CRM1 in human) recognizes proteins with classical leucine-rich nuclear export signals (NESs) and exports them to the cytoplasm via the Ran-GTP cycle (Fornerod et al., 1997). Furthermore, Xpo1 is involved in the nuclear export of the non-coding telomerase RNA TLC1 (Wu et al., 2014), but it remains unclear if the interaction of Xpol and TLC1 is direct or mediated by an adaptor-protein, because in human, the Xpo1 homologue CRM1 can only export RNAs via the interaction with an RNA-binding adaptor protein (Ohno et al., 2000).

The second type of nuclear export is mediated by Mex67 (Tap-p15 in human). The RNA export is facilitated by the heterodimer Mex67-Mtr2. Mex67-Mtr2 is able to bind RNA directly, e.g. rRNA (Yao et al., 2007). However, for mRNA export under normal physiological conditions, the binding is mediated via several adaptor proteins, e.g. the SR proteins Hrb1, Gbp2 and Npl3 (Zander et al., 2016). At the NPC, Mex67-Mtr2 interacts with the FG-repeats of the NPC and the mRNP translocates to the cytoplasm. At the cytoplasmic site, directionality of the process is achieved by the DEAD box RNA helicase Dbp5/Rat8, which interacts with the nucleoporin Nup159/Rat7. Through ATP hydrolysis and structural remodeling, Mex67 is released from the mRNA (Folkmann et al., 2011; Kelly and Corbett, 2009; Tieg and Krebber, 2013). Additionally, Mex67 is also involved in the transport of noncoding RNAs, such as TLC1 (Wu et al., 2014).

Nuclear protein import is often induced by the recognition of NLSs. Importin $\alpha$ binds to NLScontaining proteins. This binding is performed by the ARM repeat domain of importin $\alpha$ (Conti et al., 1998). Simultaneously, importin $\alpha$ binds to importin $\beta$ via its $\mathrm{N}$-terminal domain (Görlich et al., 1996). This complex translocates to the nucleus, where it is disassembled by the association of Ran-GTP. Afterwards, importin $\alpha$ is recycled to the cytoplasm through the export by Cse1 (CAS in human) (Hood and Silver, 1998). Cse1 can bind to importin $\alpha$ in the presence of Ran-GTP. In the cytoplasm the complex is released by the Rna1-induced hydrolysis of Ran-GTP (Cook et al., 2005). 


\subsection{The life cycle of snRNAs in human}

The life cycle of snRNAs in human cells differs from the snRNA lifecycle in S. cerevisiae at some steps.

Similar to yeast snRNAs, human snRNAs are also transcribed by RNAP II. In contrast to mRNA, snRNAs contain no TATA box and enhancer regions, but they include a proximal and a distal sequence element (PSE and DSE, respectively), which are essential for transcription initiation (Matera and Wang, 2014). The RNAP II and RNAP III transcribed snRNA genes contain a similar PSE, with RNAP III snRNAs also including a TATA box (Mattaj et al., 1988). This PSE is recognized by the SNAP (snRNA activating protein) complex and GTFs (general transcription factors). The binding of both the SNAP-complex and the GEFs initiates snRNA transcription. Furthermore, snRNA promotors recruit the LEC (little elongation complex) (Figure 7) (Henry et al., 1998; Matera and Wang, 2014).

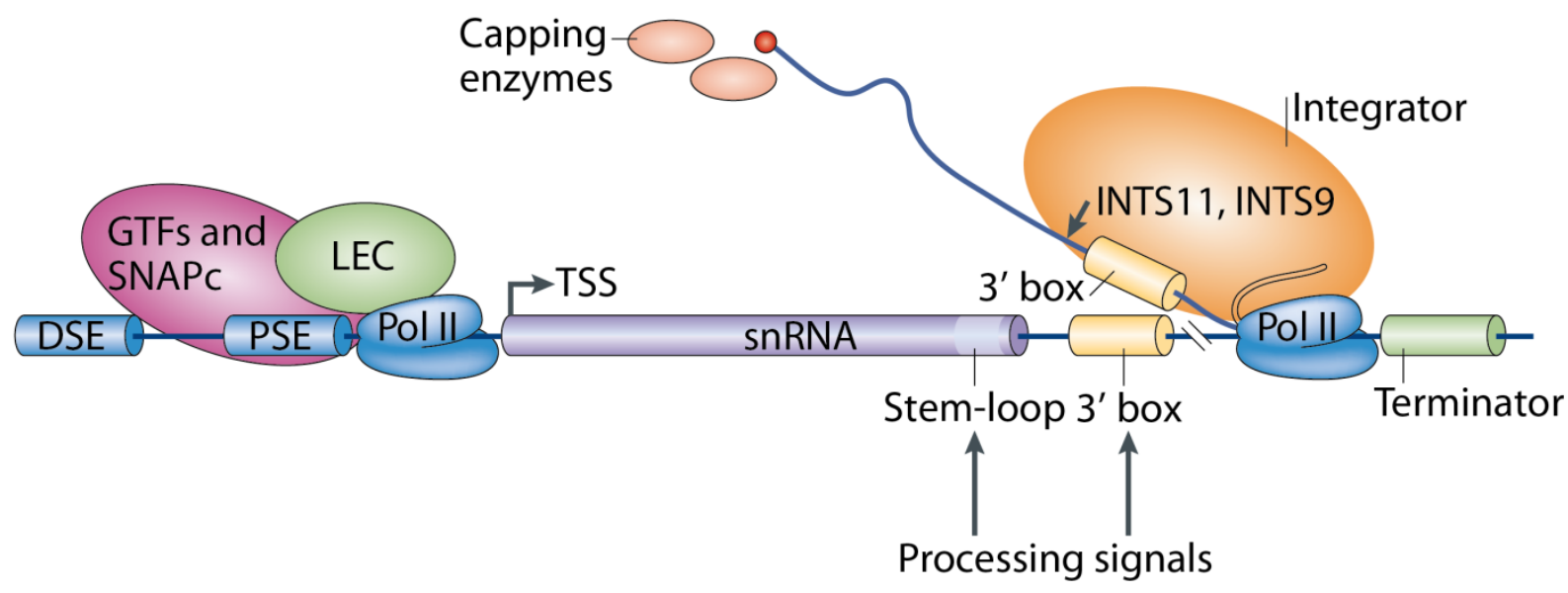

Figure 7: Transcription initiation and transcription termination of Sm-class snRNAs in human. The binding of the SNAP complex and the GTFs (general transcription factors) to the PSE initiates transcription of Sm-class snRNAs by RNAP II. Transcription termination is initiated by the Integratorcomplex, which is recruited to the transcript via RPAP2 and cuts the nascent transcript near the 3'-box downstream the 3'-stem-loop. Modified from (Matera and Wang, 2014)

In addition to the $\operatorname{Ser}_{2}$ and $\operatorname{Ser}_{5}$ phosphorylation patterns, metazoan cells show enriched $\mathrm{Ser}_{7^{-}}$ phosphorylation levels at the snRNA promotor regions and this modification appears to be important for expression regulation (Egloff, 2012; Egloff et al., 2009). There is also evidence for the necessity of $\mathrm{Ser}_{7}$-phosphorylation in snRNA transcription in HEK293 cells (Egloff et al., 2007). Phosphorylated $\mathrm{Ser}_{7}$ recruits the CTD phosphatase RPAP2 to the CTD. Then, RPAP2 dephosphorylates $\operatorname{Ser}_{5}$ and simultaneously interacts with the Integrator-complex, which is essential for transcription termination in metazoans. The Integrator-complex cuts the transcript at the 3'-box downstream of the 3'-stem-loop (Figure 8) (Baillat et al., 2005; Egloff, 
2012; Matera and Wang, 2014). How transcription termination is performed in detail remains undetermined to date.

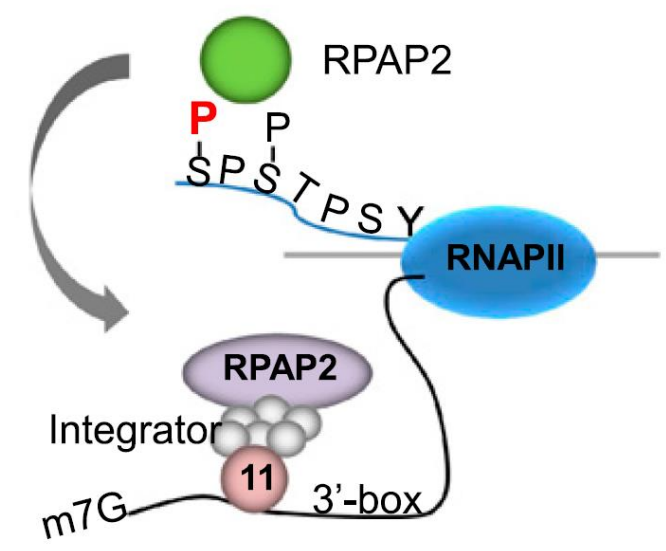

Figure 8: RPAP2 and the Integrator complex terminate Sm-class snRNA transcription. RPAP2 is recruited to the nascent transcript through its interaction with phosphorylated Ser $_{7}$. RPAP2 dephosphorylates Ser $_{5}$ and afterwards, recruits the Integrator complex, which finally cuts the transcript at its 3 '-site by an unknown mechanism. Taken from (Hsin and Manley, 2012)

Following transcription, the CBC binds to the snRNAs via its $5^{\prime}-\mathrm{m}^{7} \mathrm{G}$-cap. This interaction is essential for snRNA export in higher eukaryotes (Figure 9). In contrast, mRNA export is not affected by inhibiting CBP20 binding to the 5'-cap (Izaurralde et al., 1995). In the next step, the snRNA, with the bound $\mathrm{CBC}$, builds a pre-export complex with phosphorylated PHAX (phosphorylated adapter RNA export) (Ohno et al., 2000). Subsequently, this pre-export complex is remodeled by the recruitment of Ran-GTP bound CRM1 and exported to the cytoplasm in a Ran-mediated process very similar as described for yeast. In the cytoplasm, the export complex is released by the hydrolysis of Ran-GTP stimulated by Ran-GAP and the simultaneous dephosphorylation of PHAX by PP2A (protein phosphatase $\underline{2 \mathrm{~A}}$ ) (Kitao et al., 2008; Ohno et al., 2000).

Once in the cytoplasm, the SMN (survival of motor neurons) complex is recruited to the snRNA. The SMN complex consists of the SMN protein and several proteins called GEMINs (Matera and Wang, 2014; Zhang et al., 2011). GEMIN5 binds directly to the Sm-binding site of the snRNAs and in this way initiates the SMN complex binding to snRNAs (Battle et al., 2006). The Sm-proteins are recruited to the SMN complex by the Arg $N$-methyltransferase 5 (PRMT5), which methylates SmB, SmD1 and SmD3 (Meister et al., 2001). This arginine methylation enables the interaction of the Sm-proteins with SMN. This process is supported by pICln, which is supposed to inhibit the formation of the Sm-ring independently of the SMN complex (Figure 10) (Meister et al., 2001). According to the so called lid theory, the Sm-ring assembles in a horseshoe intermediate composed of SmD1, SmD2, SmE, SmF and 
SmG, which is then closed by a lid composed of SmB and SmD3 (Kambach et al., 1999; Kroiss et al., 2008; Matera and Wang, 2014; Zhang et al., 2011). Gemin2 simultaniously interacts with five of the Sm-ring members (SmD1, SmD2, SmE, SmF and SmG). In addition, it prevents RNA binding through extension into the RNA binding cleft of the snRNA pentamer. The fact that Gemin2 does not interact with the SmB-SmD3 heterodimer supports the lid theory. Interestingly, neurodegenerative disease spinal muscular atrophy (SMA) is normally induced by an altered SMN1 expression. Here, the mutation of the Gemin2 binding site of SMN solely results in SMA (Zhang et al., 2011).

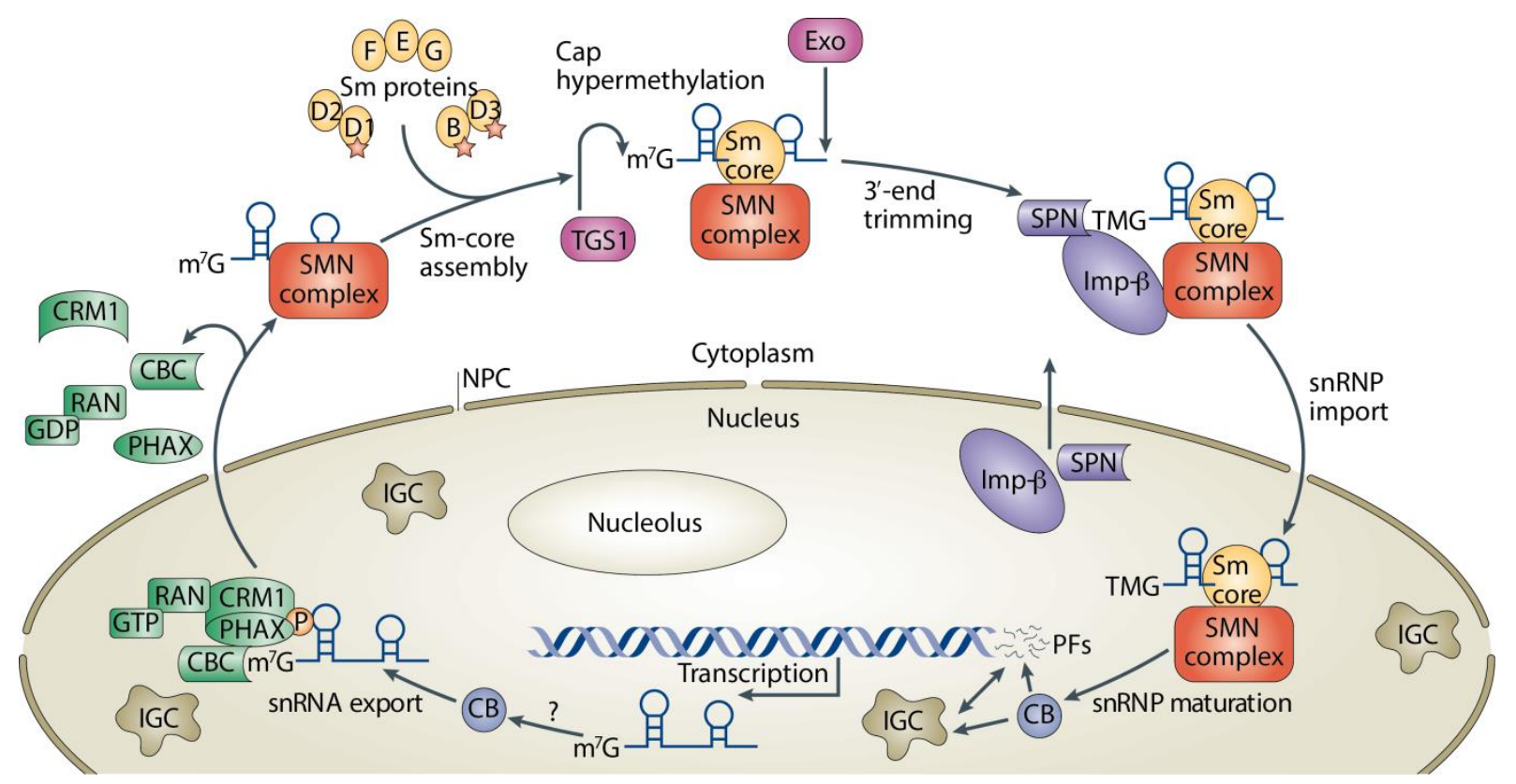

Figure 9: Model for the nucleo-cytoplasmic shuttling of snRNAs in human cells. Human snRNAs undergo a nucleo-cytoplasmic shuttling for snRNP assembly. After RNAP II transcription and cotranscriptional $m^{7} G$-capping, the snRNA export is mediated by CRM1. The CBC binds the $5^{\prime}-m^{7} G$-cap and phosphorylated PHAX is recruited. This export complex translocates to the cytoplasm where it is disassembled by RAN-GTP hydrolysis and PHAX dephosphorylation. Afterwards, the complex binds to the snRNA and promotes the Sm-ring assembly. Once the Sm-ring is assembled on the snRNA, TGS1 trimethylates the $5^{\prime}-\mathrm{m}^{7} \mathrm{G}$-cap and Snurportin 1 (SPN) can bind to the snRNP. SPN serves as an adaptor for importin $\beta$ and the snRNP is imported into the nucleus, where SPN and importin $\beta$ dissociate from the snRNP. Finally, the snRNAs are post-transcriptionally modified in Cajal bodies. Taken from (Matera et al., 2007)

Once the Sm-ring assembled on the snRNA, structural modifications of the snRNA take place. One modification is the trimethylation of the $5^{\prime}-\mathrm{m}^{7} \mathrm{G}$-cap by TGS1 (Tgs1 in yeast). TGS1 is recruited to the snRNA through the interaction with the SMN-complex and adds a 2,2,7-TMG cap (Mouaikel et al., 2003). At some point in the cytoplasmic phase, the snRNAs are 3'-processed by an unknown mechanism (Figure 9) (Matera and Wang, 2014). 


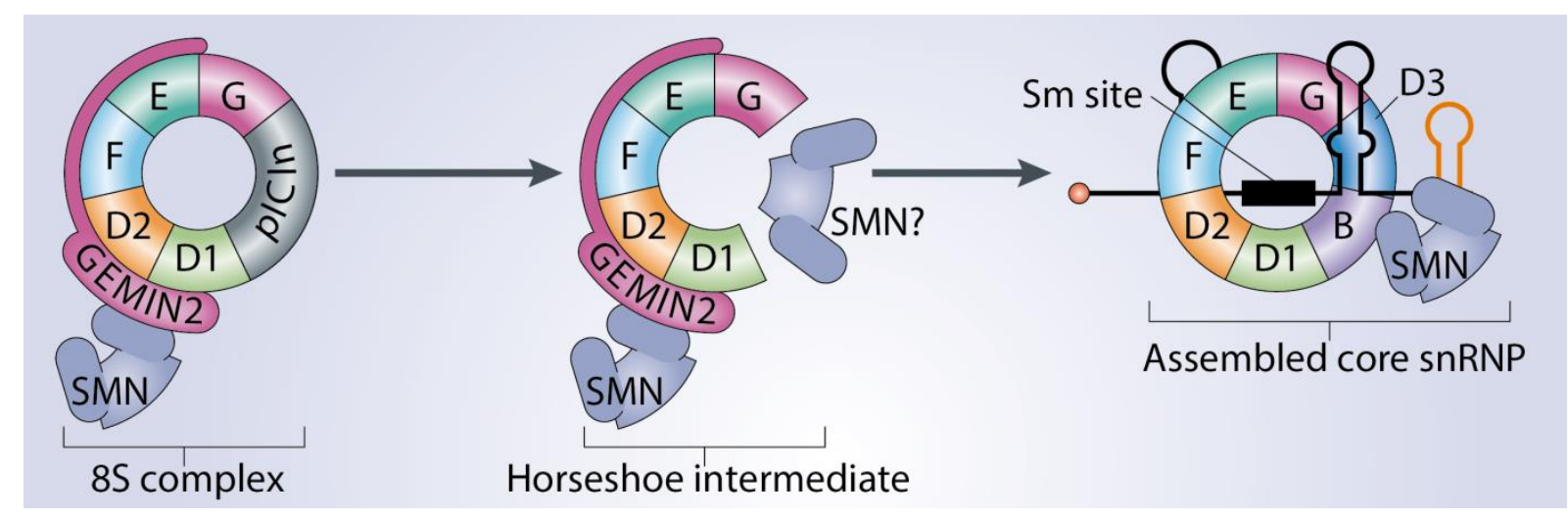

Figure 10: The Sm-ring assembles stepwise on the snRNAs following the so-called lid theory. After the recruitment of the SMN-complex and GEMIN2 to the Sm-ring pentamer (SmD1-SmD2-SmFSmE-SmG), pICln keeps the horseshoe shaped form open to prevent the complete Sm-ring assembly. Finally, the lid (SmB-SmD3) closes the Sm-ring and completes the Sm-ring assembly. Modified from (Matera and Wang, 2014).

The TMG-cap and the Sm-proteins are sufficient for nuclear import of snRNAs (Fischer and Lührmann, 1990; Fischer et al., 1993; Hamm and Mattaj, 1990). The addition of the TMG-cap to the 5' end of the snRNAs recruits Snurportin1 (SPN) (Figure 9) (Huber et al., 1998). SPN specifically recognizes TMG-caps via its C-terminal domain. In addition, the $\mathrm{N}$-terminus contains an importin $\beta$ binding (IBB) site, which is quite similar to the IBB of importin $\alpha$. Deletion of the IBB inhibits the import of Sm-class snRNAs. In contrast, the C-termini of SPN and importin $\alpha$ only display $10 \%$ sequence similarity (Huber et al., 1998). After SPN binding, the import is facilitated by importin $\beta$ through its interaction with SPN (Huber et al., 1998; Palacios et al., 1997). Finally, the import complex of the snRNP dissociates in the nucleus and snRNPs undergo final maturation steps in Cajal bodies, such as posttranscriptional methylation, pseudouridylation and binding of snRNP specific proteins (Jády et al., 2003; Matera and Wang, 2014; Nesic et al., 2004).

\subsection{SnRNA related diseases}

\subsubsection{Spinal muscular atrophy}

The SMA is an inherited neurodegenerative condition effecting the spinal motor neurons. The degeneration of the spinal motor neurons results in the loss of mobility and can lead, in its most severe form, to infant mortality. With a mortality rate of one in 6,000 cases, SMA is the most common cause for infant mortality (Sleeman, 2013). The manifestation of SMA is related to SMN. Patients suffering from SMA exhibit insufficient amounts of SMN. Nearly $99 \%$ of the patients are either lacking the SMN1 gene or carry a truncated form, while the remaining patients have point mutations in the gene (Lefebvre et al., 1995, 1997). The absence of the correct SMN1 transcription is counterbalanced by the expression of the SMN2 
gene. Due to alternative splicing, the expression of SMN2 results in a truncated form of SMN. It is speculated that embryonic cells can compensate the missing SMN from SMN1 expression by the truncated version of SMN expressed by SMN2. In contrast, small children cannot compensate the truncated SMN anymore, which finally leads to death (Ahmad et al., 2016). Furthermore, it is known that both genes (SMN1 and SMN2) have the same promoter sequence and are highly expressed in neuronal cells. The reason why especially neuronal cells are affected is still unclear (Boda et al., 2004). Taken together, the exact pathomechanism of SMA is still unknown (Ahmad et al., 2016).

\subsubsection{U6 related diseases}

C16orf57 is the human homologue to the yeast U6 phosphodiesterase Usb1 (see 2.2.2), which is essential for U6 3'-processing and its stability (Mroczek et al., 2012). Interestingly, mutations of C16orf57 are found in several diseases, e.g. poikiloderma with neutropenia, the Rothemund-Thomson syndrome (RTS) and dyskeratosis congenital (DC) disease. RTS and DC are also caused by mutations in genes involved in DNA repair and telomere maintenance. While the depletion of yeast Usb1 results in the degradation of U6 and severe splicing defects, mutations in C16orf57 lead to extended poly-uridenylated U6, but surprisingly no splicing defects. Diseases caused by C16orf57 mutations are severe but not lethal indicating that the false processing of U6 may have a more defined effect on splicing. But the reason for that still needs to be elucidated (Mroczek et al., 2012). 


\subsection{Aim of the study}

Splicing is a molecular process characteristic for eukaryotic cells. It has enabled the possibility of alternative splicing in higher eukaryotes. Splicing is performed by the spliceosome consisting of five spliceosomal subunits. Each of these five subunits contains exactly one snRNA. Prior to spliceosome assembly, the snRNAs have to undergo several processing steps and acquire an Sm- or an Lsm-ring. In human cells, these processing steps and the snRNP assembly are cytoplasmic processes and thus separated from the place of spliceosomal assembly and activity (Matera and Wang, 2014). It is still not known, why such a separation process has evolved. A possible quality control mechanism for RNP assembly was suggested but has never been shown so far (Matera and Wang, 2014; Sloan et al., 2016).

The major goal of this study is to investigate snRNA shuttling in yeast and to tackle the question, why nucleo-cytoplasmic shuttling of snRNAs was evolutionary established. 


\section{Materials and Methods}

\subsection{Chemicals and Consumables}

Table 1: Chemicals and materials used in this study

\begin{tabular}{|c|c|}
\hline Chemical / Consumable & Supplier / Source \\
\hline $1 \mathrm{ml} \mathrm{GSTrap}{ }^{\mathrm{TM}} \mathrm{FF}$ & GE Healthcare (Freiburg/Germany) \\
\hline Agarose NEEO Ultra & Carl Roth (Karlsruhe/Germany) \\
\hline Amersham Protran $0.45 \mu \mathrm{m}$ nitrocellulose membrane & GE Healthcare (Freiburg/Germany) \\
\hline Complete EDTA-free protease inhibitor & Roche (Mannheim/Germany) \\
\hline $\mathrm{DC}^{\mathrm{TM}}$ protein assay & BioRad (München/Germany) \\
\hline Deionized Formamide & Applichem (München/Germany) \\
\hline dNTPs & Thermo Fischer Scientific (Schwerte/Germany) \\
\hline 5-Fluoroortic acid & Apollo Scientific (Derbyshire/UK) \\
\hline Formaldehyde $37 \%$ & AppliChem (München/Germany) \\
\hline GFP-Trap beads & ChromoTek (Planegg-Martinsried/Germany) \\
\hline GoTaq® qPCR Master Mix & Promega (Mannheim/Germany) \\
\hline GSTrap FF & GE Healthcare (Freiburg/Germany) \\
\hline IgG Sepharose 6 FastFlow & GE Healthcare (Freiburg/Germany) \\
\hline Microscope slides, 12 well, $5.2 \mathrm{~mm}$, PTFE-coating & Thermo Fischer Scientific (Schwerte/Germany) \\
\hline Phenol/chloroform/isoamyl alcohol (25:24:1) & Carl Roth (Karlsruhe/Germany) \\
\hline Poly-L-lysine hydrobromide & Sigma-Aldrich (München/Germany) \\
\hline qPCRBIO SyGreen Mix Lo-ROX & Nippon Genetics (Düren/Germany) \\
\hline RiboLock RNase Inhibitor & Thermo Fischer Scientific (Schwerte/Germany) \\
\hline tRNAs & Sigma-Aldrich (München/Germany) \\
\hline Rotiphorese Gel 30 (37.5:1) acrylamide & Carl Roth (Karlsruhe/Germany) \\
\hline Salmon Sperm DNA & Sigma-Aldrich (München/Germany) \\
\hline SiLibeads type S 0.4-0.6 mm & Th. Geyer (Renningen/Germany) \\
\hline Trizol® Reagent & Life Technologies (Darmstadt/Germany) \\
\hline Vivaspin 20 & VivaScience, Sartorius Group (Göttingen, Germany) \\
\hline Whatman® Blotting Paper $0.8 \mathrm{~mm}$ & Hahnemühle (Dassel/Germany) \\
\hline
\end{tabular}

Table 2: Kits used in this study

\begin{tabular}{l|l}
\hline Kit & Supplier / Source \\
\hline $\begin{array}{l}\text { Amersham ECL Prime Western Blotting Detection } \\
\text { Kit }\end{array}$ & GE Healthcare (Freiburg/Germany) \\
\hline DIG RNA labeling mix, 10x & Roche (Mannheim/Germany) \\
\hline NucleoSpin Gel and PCR Clean-up & Macherey-Nagel (Düren/Germany) \\
\hline NucleoSpin Plasmid & Macherey-Nagel (Düren/Germany) \\
\hline NucleoSpin RNA & Macherey-Nagel (Düren/Germany) \\
\hline PageSilver Silver staining Kit & Fermentas (Waltham/USA) \\
\hline peqGOLD Gel Extraction Kit & Peqlab (Erlangen/Germany) \\
\hline WesternBright Chemilumineszenz Substrate & Biozym (Hess.Ohlendorf/Germany) \\
Quantum &
\end{tabular}


Table 3: Marker and standards used in this study

\begin{tabular}{l|l}
\hline Marker / Standard & Supplier / Source \\
\hline GeneRuler 100bp DNA Ladder & Thermo Fischer Scientific (Schwerte/Germany) \\
\hline Lambda DNA/EcoRI + HindIII Marker & Thermo Fischer Scientific (Schwerte/Germany) \\
\hline PageRuler Prestained Protein Ladder & Thermo Fischer Scientific (Schwerte/Germany) \\
\hline
\end{tabular}

Chemicals, consumables or other material that are not specifically stated above were purchased from the companies listed below:

AppliChem (München/Germany), BD Biosciences (Heidelberg/Germany), Carl Roth (Karlsruhe/Germany), GE Healthcare (Freiburg/Germany), Life Technologies (Darmstadt/Germany), Merck (Darmstadt/Germany), New England Biolabs (Frankfurt a.M./Germany), OMNILAB GmbH (Bremen/Germany), Peqlab (Erlangen/Germany), Promega (Mannheim/Germany), Roche (Mannheim/Germany), Sarstedt (Nürnbrecht/Germany), Serva (Heidelberg/Germany), Sigma-Aldrich (München/Germany), Thermo Fischer Scientific (Schwerte/Germany), Th.Geyer (Renningen/Germany), VWR (Darmstadt/Germany)

\subsection{Equipment and Hardware}

Table 4: Equipment and hardware used in this study

\begin{tabular}{l|l}
\hline Machine & Supplier / Source \\
\hline Äkta Prime plus & GE Healthcare (Freiburg/Germany) \\
\hline Cell homogenizer FastPrep-24 & MP Biomedicals (Illkirch/France) \\
\hline $\begin{array}{l}\text { Centrifuge Heraeus Multifuge X3R with swinging bucket } \\
\text { rotor TX-750 or Fiberlite F15-8x50cy fixed-angle rotor }\end{array}$ & Thermo Fischer Scientific (Schwerte/Germany) \\
\hline Centrifuge Heraeus Fresco21 & Thermo Fischer Scientific (Schwerte/Germany) \\
\hline Eclipse E400 tetrad microscope & Nikon (Düsseldorf/Germany) \\
\hline Electro Blotter PerfectBlue Semi-Dry, Sedec M & Peqlab (Erlangen/Germany) \\
\hline $\begin{array}{l}\text { Fluorescence microscope DMI6000B with Leica DFC360 } \\
\text { FX camera }\end{array}$ & Leica (Wetzlar/Germany) \\
\hline Hemocytometer Neubauer improved & Carl Roth GmbH (Karlsruhe/Germany) \\
\hline INTAS UV-System for Gel detection & INTAS GmbH (Göttingen/Germany) \\
\hline Luminescence Detection System Fusion SL 3500.WL & Peqlab (Erlangen/Germany) \\
\hline LM10 Microfluidizer & Microfluidics (Westwood, USA) \\
\hline NanoDrop 2000 spectrophotometer & Thermo Fischer Scientific (Schwerte/Germany) \\
\hline qPCR Cycler CFX Connect & BioRad (München/Germany) \\
\hline qPCR Cycler Rotor-Gene Q & Qiagen (Hilden/Germany) \\
\hline Sorvall WX Ultra Series Centrifuge & Thermo Fischer Scientific (Schwerte/Germany) \\
\hline Ti50 Rotor & Beckmann Coulter (Krefeld/Germany) \\
\hline Thermocycler MyCycler & BioRad (München/Germany) \\
\hline Water Purification Milli-Q & Millipore (Eschborn/Germany) \\
\hline
\end{tabular}




\subsection{Software}

Table 5: Software used in this study

\begin{tabular}{l|l}
\hline Software & Supplier / Source \\
\hline Adobe Illustrator CS5; Adobe Photoshop CS5 & Adobe Systems (San Jose/USA) \\
\hline ApE Plasmid Editor & M.Wayne Davis (University of Utah/USA) \\
\hline Fiji (1.48s) & W. Rasband (NIH/USA) \\
\hline GraphPad Prism 5 & GraphPad Software (San Diego/USA) \\
\hline Microsoft Office 2010 & Microsoft Corporation (Redmond/USA) \\
\hline Microscopy LAS AF 1.6.2 & Leica (Wetzlar/Germany) \\
\hline
\end{tabular}

\subsection{Antibodies and Enzymes}

Table 6: Antibodies used in this study

\begin{tabular}{l|l|l}
\hline Antibody (organism) & Dilution & Supplier / Source \\
\hline $\begin{array}{l}\text { Anti-Digoxigenin-FITC, } \\
\text { Fab fragments (sheep) }\end{array}$ & $1: 40$ (FISH) & Roche (Mannheim/Germany) \\
\hline Anti-GFP (GF28R) (mouse) & $1: 4,000$ & Thermo Fischer Scientific (Schwerte/Germany) \\
\hline Anti-GST (B-14) (mouse) & $1: 2,000$ & Santa Cruz (Heidelberg/Germany) \\
\hline Anti-Hem15 & $1: 10,000$ & courtesy of Prof. Roland Lill (Marburg/Germany) \\
\hline Anti-mouse IgG-HRP (goat) & $1: 10,000$ & Dianova (Hamburg/Germany) \\
\hline Anti-myc (A-14) (rabbit) & $1: 1,000$ & Santa Cruz (Heidelberg/Germany) \\
\hline Anti-Nop1 (mouse) & $1: 1,000$ & Santa Cruz (Heidelberg/Germany) \\
\hline Anti-rabbit IgG-HRP (goat) & $1: 10,000$ & Dianova (Hamburg/Germany) \\
\hline Anti-Zwf1 (rabbit) & $1: 20,000$ & courtesy of Prof. Roland Lill (Marburg/Germany) \\
\hline
\end{tabular}

Table 7: Enzymes used in this study

\begin{tabular}{l|l}
\hline Enzyme & Supplier / Source \\
\hline Benzonase & Merck (Darmstadt/Gemany) \\
\hline RNase-free DNase & Qiagen (Hilden/Germany) \\
\hline DreamTaq DNA polymerase & Thermo Fischer Scientific (Schwerte/Germany) \\
\hline FastAP Alkaline Phosphatase & Thermo Fischer Scientific (Schwerte/Germany) \\
\hline Lysozyme & Thermo Fischer Scientific (Schwerte/Germany) \\
\hline Maxima Reverse Transcriptase & Thermo Fischer Scientific (Schwerte/Germany) \\
\hline Phusion High fidelity & Thermo Fischer Scientific (Schwerte/Germany) \\
\hline Q5 DNA polymerase & New England Biolabs (Frankfurt/Germany) \\
\hline Restriction Enzymes & Thermo Fischer Scientific (Schwerte/Germany) \\
\hline Restriction Enzymes & New England Biolabs (Frankfurt/Germany) \\
\hline RNase A & Qiagen (Hilden/Germany) \\
\hline T4 DNA Ligase & Thermo Fischer Scientific (Schwerte/Germany) \\
\hline T5 Exonuclease & New England Biolabs (Frankfurt/Germany) \\
\hline Taq DNA Ligase & New England Biolabs (Frankfurt/Germany) \\
\hline Zymolyase 20T & Amsbio (Abingdon/UK) \\
\hline
\end{tabular}




\subsection{Strains}

\subsubsection{Escherichia coli strains}

Table 8: $E$. coli strains used in this study

\begin{tabular}{l|l|l}
\hline Strain & Genotype & Application \\
\hline DH5 $\alpha^{\mathrm{TM}}$ & $\begin{array}{l}\text { F- } \Phi 80 \text { lacZ } \Delta \text { M15 } \Delta(\text { lacZYA-argF) U169 recA1 endA1 hsdR17 } \\
(\mathrm{rK}-, \text { mK+) phoA supE44 } \lambda-\text { thi-1 gyrA96 relA1 }\end{array}$ & plasmid amplification \\
\hline BL21*(DE3) & F- ompT hsdSB (rB-mB-) gal dem rne131 (DE3) & protein expression \\
\hline
\end{tabular}

\subsubsection{Saccharomyces cerevisiae strains}

Table 9: S. cerevisiae strains used in this study

\begin{tabular}{|c|c|c|}
\hline Number & Genotype & Source \\
\hline HKY36 & 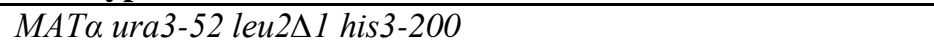 & (Winston et al., 1995) \\
\hline HKY37 & MAT $\alpha$ ura3 leu2 trp1 his3 ade2 srp1-31 & (Loeb et al., 1995) \\
\hline HKY46 & MATa ura3-52 lys2-301 ade2 mtr10-1 & (Liu et al., 1999) \\
\hline HKY62 & MATa ura leu his msn5::HIS3 & Laboratory of Heike Krebber \\
\hline HKY74 & MATa leu his lys ade lph2::HIS3 & Laboratory of Heike Krebber \\
\hline HKY82 & MAT $\alpha$ ura 3 leu 2 trp his3 ade 2 mtr10::HIS3 & (Senger et al., 1998) \\
\hline HKY83 & MAT $\alpha$ ura leu trp his ade nmd5::HIS3 & Laboratory of Heike Krebber \\
\hline HKY86 & MATa ura leu his lys kap104::HIS3 & Laboratory of Heike Krebber \\
\hline HKY124 & 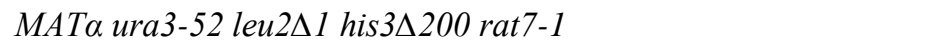 & (Gorsch et al., 1995) \\
\hline HKY130 & MATa ura3-52 leu $2 \Delta 1$ trp $1 \Delta 63$ rat $8-2$ & (Snay-Hodge et al., 1998) \\
\hline HKY133 & 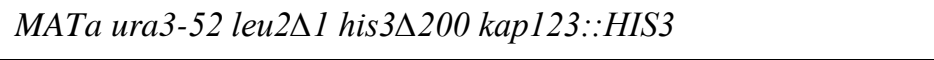 & (Seedorf and Silver, 1997) \\
\hline HKY137 & 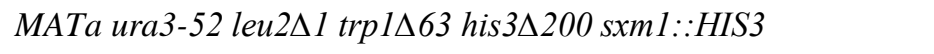 & (Seedorf and Silver, 1997) \\
\hline HKY138 & MATa ura3-52 leu $2 \Delta 1$ trp $1 \Delta 63$ pse $1-1$ & (Seedorf and Silver, 1997) \\
\hline HKY149 & 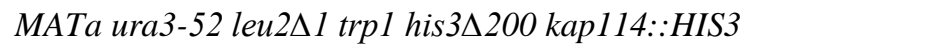 & (Pemberton et al., 1999) \\
\hline HKY204 & MATа ura3-52 trp $1 \Delta 63$ leu $2 \Delta 1$ pse1-21 & (Seedorf and Silver, 1997) \\
\hline HKY206 & $\begin{array}{l}\text { MAT } \alpha \text { ade2-1 his leu trp1-1 ura3-1 xpol::LEU2 } \\
+p \text { CEN TRP1 xpo1-1 }\end{array}$ & (Taura et al., 1998) \\
\hline HKY208 & MAT $\alpha$ ura3-52 ade2-101 his3-11,15, trp1- $\Delta 901$ cse1-1 & (Xiao et al., 1993) \\
\hline HKY209 & MATa lys2 trp1 ura3-52 leu2A1 pdr6::HIS3 & (Titov and Blobel, 1999) \\
\hline HKY270 & MAT $\alpha$ ura3 leu 2 his3 ade 2 mtrl0 $: H I S 3+p$ CEN TRP1 mtrl0-7 & (Senger et al., 1998) \\
\hline HKY381 & MAT $\alpha$ ura $3 \Delta 0$ leu $2 \Delta 0$ his $3 \Delta 1$ lys $2 \Delta 0$ & $\begin{array}{l}\text { Euroscarf } \\
\text { (Oberursel/Germany) }\end{array}$ \\
\hline HKY644 & $\begin{array}{l}\text { MAT } \alpha \text { ura3 leu2 trp1 his3 ade2 mex67::HIS3 } \\
+p \text { CEN LEU2 mex67-5 }\end{array}$ & (Segref et al., 1997) \\
\hline HKY680 & MATa ade, his7, ura3-52, lys2 rna6-1 & (Legrain and Rosbash, 1989) \\
\hline HKY1193 & 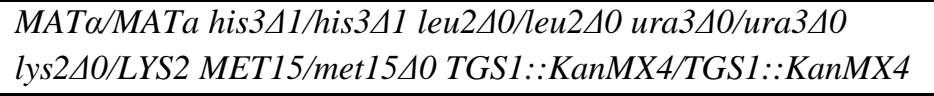 & $\begin{array}{l}\text { Euroscarf } \\
\text { (Oberursel/Germany) }\end{array}$ \\
\hline HKY1266 & 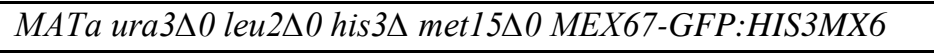 & Invitrogen (Carlsbad/USA) \\
\hline HKY1277 & 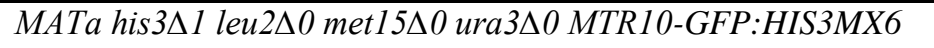 & Invitrogen (Carlsbad/USA) \\
\hline HKY1305 & 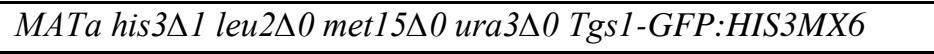 & Invitrogen (Carlsbad/USA) \\
\hline
\end{tabular}


3 Materials and Methods

\begin{tabular}{|c|c|c|}
\hline HKY1353 & $\begin{array}{l}\text { MATa ura3-52 mex67::HIS3 xpo1::TRP1 xpo1-1::HIS } \\
+p \text { CEN LEU2 mex67-5 }\end{array}$ & (Brune et al., 2005) \\
\hline HKY1377 & 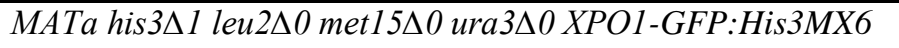 & Invitrogen (Carlsbad/USA) \\
\hline HKY 1576 & $\begin{array}{l}\text { MATa trp1-1 ura3-1 his3-11,15 leu2-3,112 can1-100 } \\
\text { ade2-1::ADE2:ura3 kap95::kap95-E126K }\end{array}$ & (Ryan et al., 2007) \\
\hline HKY1596 & 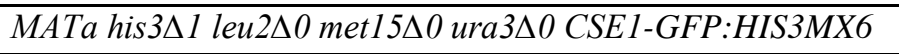 & Invitrogen (Carlsbad/USA) \\
\hline HKY1642 & $\begin{array}{l}\text { MATa ura3-52 lys2-801 ade2-D trp1-D63 his3-D200 leu2-D1 } \\
\text { smb::KanMX4 } \\
+p P_{G A L l}: \text { TRP-SmB }\end{array}$ & (Bordonné, 2000) \\
\hline HKY1645 & $\begin{array}{l}\text { MATa ura3-52 lys2-801 ade2-D trp1-D63 his3-D200 leu2-D1 } \\
\text { smb::KanMX4 smd1::LEU2 } \\
+p \text { GFP-HIS3-SmD1 } 119-125+p P_{G A L 1}: \text { TRP-SmB }\end{array}$ & (Bordonné, 2000) \\
\hline HKY1700 & 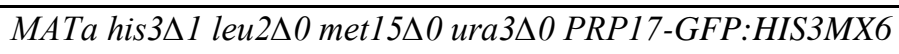 & Invitrogen (Carlsbad/USA) \\
\hline HKY1720 & 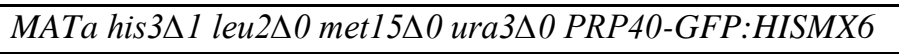 & Invitrogen (Carlsbad/USA) \\
\hline HKY1732 & MATa his3D1 ade2 trp1 PRP40-GFP:HISMX6 mex67::HIS3 & $\begin{array}{l}\text { this study } \\
\text { (HKY644xHKY1720) }\end{array}$ \\
\hline
\end{tabular}

\subsection{Plasmids}

Table 10: Plasmids used in this study

\begin{tabular}{|c|c|c|}
\hline Number & Features & Source \\
\hline pHK12 & CEN URA3 $P_{A D H}: N L S-N E S-G F P-G F P$ & (Taura et al., 1998) \\
\hline pHK88 & CEN URA3 & (Sikorski and Hieter, 1989) \\
\hline pHK750 & CEN URA3 $P_{A D H}: N L S-N E S-M Y C-M Y C-M Y C$ & Laboratory of Heike Krebber \\
\hline pHK1288 & $p G E X-6 P-1$ & $\begin{array}{l}\text { GE Healthcare } \\
\text { (Freiburg/Germany) }\end{array}$ \\
\hline pHK1372 & $P_{T R C}: H I S: T E V: M T R 2: M E X 67$ & (Yao et al., 2007) \\
\hline pHK1373 & $P_{T R C}: H I S: T E V: M T R 2:$ mex67loopKR $>A A$ & (Yao et al., 2007) \\
\hline pHK1374 & $P_{T R C}: H I S: T E V: M T R 2:$ mex67-409-435aaK343E & (Yao et al., 2007) \\
\hline pHK1469 & CEN URA SmB-GFP & this study from $\mathrm{pHK} 12$ \\
\hline pHK1476 & $p G E X-6 P-1$ AmpR $P_{\text {tac }}: G S T-C s e 1$ & this study from $\mathrm{pHK} 1288$ \\
\hline pHK1480 & CEN URA SmG-GFP & this study from pHK1469 \\
\hline pHK1482 & CEN URA SmB-3xmyc & this study from pHK750 \\
\hline pHK1574 & CEN URA Cbp80-3xmyc & this study from $\mathrm{pHK} 750$ \\
\hline
\end{tabular}

\subsection{Oligonucleotides}

Table 11: Oligos used in this study

\begin{tabular}{|c|c|c|}
\hline Number & Sequence & Name \\
\hline HK778 & 5'-GGTCCGTGTTTATGATGG-3' & $E F B 1$ intron forward \\
\hline HK779 & 5'-CTCTGCTCTGTGCTATCG-3' & $E F B 1$ intron reverse \\
\hline HK800 & 5'-CGCTGAAGCTGAAAAGTTG-3' & $E F B 1$ forward \\
\hline HK801 & 5'-CCAATTGGGATAAATTGGTGAG-3' & EFB1 reverse \\
\hline HK839 & 5'-GGTATCGAATCCAAGCAACC-3' & $R P S 23 A$ forward \\
\hline HK840 & 5'-GCTTTTCCTTCTTTTCTTTCCAC-3' & $R P S 23 A$ reverse \\
\hline HK842 & 5'-CCAAGAACGTTTCTTGTTACAGACC-3' & RPS6A forward \\
\hline HK843 & 5'-CGTCATCTTCCTTGGACAAACC-3' & RPS6A reverse \\
\hline HK874 & 5'-TCACCAACGGCTTCACCATC-3' & $R P S 6 A$ intron reverse \\
\hline
\end{tabular}


3 Materials and Methods

\begin{tabular}{|c|c|c|}
\hline HK1093 & 5'-GGTTTACATATACTGTTACTACAAACC-3' & RPS6A intron forward \\
\hline HK1404 & 5'-TCGCGAAGTAACCCTTCGTG-3' & SNR6 forward \\
\hline HK1405 & 5'-AAACGGTTCATCCTTATGCAGG-3' & SNR6 reverse \\
\hline HK1492 & $\begin{array}{l}\text { 5'-TAATAGGACTCACTATAGGGAAATAAATCTCTTTGT } \\
\text { AAAACGGTTCATCC-3' }\end{array}$ & SNR6 reverse $+\mathrm{T} 7$ site \\
\hline HK1608 & 5'-TATCAGAGGAGATCAAGAAGTCCTA-3' & SNR19 forward \\
\hline HK1609 & 5'-TAATAGGACTCACTATAGGGAACGAGCAAAGTTGAGA-3' & $\begin{array}{l}\text { SNR19 reverse + T7 } \\
\text { site }\end{array}$ \\
\hline HK1628 & 5'-CTATCAACCCCCTATTGTGATATCG-3' & $R P S 23 A$ intron forward \\
\hline HK1629 & 5'-GATTGGGAAGAATGGCCGAAGC-3' & $R P S 23 A$ intron reverse \\
\hline HK1630 & 5'-CATCTCCAATAAACATGTATGCAAG-3' & $R P L 21 B$ intron forward \\
\hline HK1631 & 5'-GCACTTGCTTTGCTCACTAC-3' & $R P L 21 B$ intron reverse \\
\hline HK1723 & 5'-CGAGCCGTTTATGTCCAACG-3' & LSRI forward \\
\hline HK1724 & 5'-TAATAGGACTCACTATAGGGCCGATACTTGGGGGATAAG-3' & LSRI reverse $+\mathrm{T} 7$ site \\
\hline HK1738 & 5'-TGCAAACTCCTTGGTCACAC-3' & SNR19 forward \\
\hline HK1739 & 5'-CCAGGCAGAAGAAACAAAGG-3' & SNR19 reverse \\
\hline HK1740 & 5'-AGCCATGACTGCATCTGTTG-3' & LSR1 forward \\
\hline HK1741 & 5'-ACAGGCGTCAACCATCAAG-3' & LSRI reverse \\
\hline HK1764 & $\begin{array}{l}\text { 5'-Су3-AGAATGCCTCTACAAAGCTCTCTTTTGAAAGGCCC } \\
\text { CAGCTCCССТАACAC-Су3-3' }\end{array}$ & SNR19 \\
\hline HK1765 & $\begin{array}{l}\text { 5'-Су3-CGGTTCATCCTTATGCAGGGGAACTGCTGA } \\
\text { TCATCTCTGTATTGTTTCAA-Сy3-3' }\end{array}$ & SNR6 \\
\hline HK1766 & $\begin{array}{l}\text { 5'-Cy3-ATGAGCGAAGAAAAATAGCAGCAAAAAGTA } \\
\text { GTGAGACCTGACATTAGCGG-Cy3-3' }\end{array}$ & LSRI \\
\hline HK1767 & $\begin{array}{l}\text { 5'-Су3-TTAACAATGGAACACTCTTATTGTAGACCAA } \\
\text { ССССАСССТАСАСССССТС-Су3-3' }\end{array}$ & LSRI \\
\hline HK1861 & $\begin{array}{l}\text { 5'-ATTO488-GCCGCAGTTGGTAAAACCTAAAACGACCGTACT } \\
\text { TGCATTATACCTCAAGCACGCAGAGAAACCTCTCTTTGG- } \\
\text { ATTO488-3' }\end{array}$ & ITS2 \\
\hline HK2040 & $\begin{array}{l}\text { 5'-ACTCACTATAGGGCGAATTGGAGCTCTCAATG } \\
\text { CGTCTAACTTGGC-3' }\end{array}$ & pHK1469 forward \\
\hline HK2041 & $\begin{array}{l}\text { 5'-TTCTCCTTTGCTAGCCATAGCTCGAGGTTTTCT } \\
\text { TTTAAAACCTGGTGGGG-3' }\end{array}$ & pHK1469 reverse \\
\hline HK2124 & 5'-TTCCAGGGGCCCCTGGGATCCATGTCCGATTTGGAAACC-3' & pHK1476 forward \\
\hline HK2125 & $\begin{array}{l}\text { 5'-CGATGCGGCCGCTCGAGTTAATTACCAACTAATA } \\
\text { ATTGATTTAATTTTAC-3' }\end{array}$ & pHK1476 reverse \\
\hline HK2164 & $\begin{array}{l}\text { 3'-ACTCACTATAGGGCGAATTGGAGCTCCGAGG } \\
\text { TGAGTCAAAGGGTAC-5' }\end{array}$ & pHK1480 forward \\
\hline HK2165 & $\begin{array}{l}\text { 3'-TCTCCTTTGCTAGCCATAGCTCGAGGTATGGCATC } \\
\text { TAGAGCCTCTAGG-5' }\end{array}$ & pHK1480 reverse \\
\hline HK2178 & $\begin{array}{l}\text { 5'-ACTCACTATAGGGCGAATTGGAGCTCTCAATGC } \\
\text { GTCTAACTTGGC-3' }\end{array}$ & pHK1482 forward \\
\hline HK2179 & $\begin{array}{l}\text { 5'-ACTTTTGTTCACCTCTAGAGGAATTaCGCGTTTTT } \\
\text { CTTTTAAAACCTGGTGGG-3' }\end{array}$ & pHK1482 reverse \\
\hline HK2271 & 5'-GACGGCAGATTCGAATGAACT-3' & $\begin{array}{l}\text { SNR19 unprocessed } \\
\text { forward }\end{array}$ \\
\hline HK2275 & 5'-GTCTTGTCTCAATGTAGCG-3' & $\begin{array}{l}\text { SNR19 unprocessed } \\
\text { reverse }\end{array}$ \\
\hline HK2507 & $\begin{array}{l}\text { 5'-ACTCACTATAGGGCGAATTGGAGCTGACGAT } \\
\text { TCTTCGAGTGTGTC-3' }\end{array}$ & pHK1574 forward \\
\hline
\end{tabular}


3 Materials and Methods

\begin{tabular}{l|l|l}
\hline HK2508 & $\begin{array}{l}\text { 5'-ACTTTTGTTCACCTCTAGAGGAATTAACTTCCTT } \\
\text { TGTTTCTTGAATCC-3' }\end{array}$ & pHK1574 reverse \\
\hline HK2652 & 5'-CGTTACCAAGTCTTCTGTTGG-3' & RPL21B forward \\
\hline HK2653 & 5'-GAGGAACGTTACCTTCAGTAG-3' & RPL21B reverse \\
\hline
\end{tabular}

\subsection{Cell cultivation}

All media were autoclaved before use and heat sensitive components, such as antibiotics and galactose were sterile-filtered and added after autoclaving. Plates were prepared by the addition of $1.5 \%$ agar for E. coli or $1.8 \%$ agar for S. cerevisiae to the liquid medium.

\subsubsection{Cultivation of $E$. coli}

Cultivation of E. coli cells was performed according to (Sambrook et al., 1989). With the exception of the synthesis of recombinant protein, LB medium was used for cultivation of $E$. coli cells. For liquid culture, the medium was inoculated with a single colony and incubated at $37{ }^{\circ} \mathrm{C}$ with agitation at $\sim 160 \mathrm{rpm}$. For selection of resistance genes, the respective antibiotics were added to the medium.

For the purification of recombinant protein, $5 \mathrm{ml}$ liquid medium with the selective antibiotic were inoculated with several colonies from a selective LB-plate and incubated over night at $37^{\circ} \mathrm{C}$. The overnight culture was added to 21 autoinducing medium (see 3.12.1), which was then incubated at $16{ }^{\circ} \mathrm{C}$ for $72 \mathrm{~h}$. Afterwards, the cells were harvested, frozen in liquid nitrogen and finally stored at $-20^{\circ} \mathrm{C}$.
LB medium $\mathrm{pH} 7.5$
$1.0 \%(\mathrm{w} / \mathrm{v})$ Peptone
$0.5 \%(\mathrm{w} / \mathrm{v})$ Yeast extract
$0.5 \%(\mathrm{w} / \mathrm{v}) \mathrm{NaCl}$

Antibiotic concentration

$$
\begin{array}{ll}
\text { Ampicillin } & 100 \mu \mathrm{g} / \mathrm{ml} \\
\text { Kanamycin } & 20 \mu \mathrm{g} / \mathrm{ml}
\end{array}
$$

\subsubsection{Cultivation of $S$. cerevisiae}

Yeast cells were cultivated in either full medium with glucose (YPD), in full medium with galactose (YP-Gal) or in selective media according to standard protocols (Sherman, 2002).

YPD medium

$2.0 \%(w / v)$ Peptone

$1.0 \%(\mathrm{w} / \mathrm{v})$ Yeast extract

$2.0 \%(\mathrm{w} / \mathrm{v})$ Glucose 
YP-Gal medium

$2.0 \%(\mathrm{w} / \mathrm{v})$ Peptone

$1.0 \%(\mathrm{w} / \mathrm{v})$ Yeast extract

$2.0 \%(w / v)$ Galactose

Selective medium

$0.2 \%(\mathrm{w} / \mathrm{v})$ Yeast drop out mix

$0.17 \%(w / v)$ Yeast nitrogen base

$0.5 \%(w / v)$ Ammonium sulfate

$2.0 \%(\mathrm{w} / \mathrm{v})$ Glucose

Cells were grown in full medium (YPD or YP-Gal), if no selection was necessary.

The yeast drop out mix of the selective medium consists of every amino acid except the one resulting from the metabolic product of the corresponding marker gene. The selective media were used for the selection of strains with genetic markers that cause metabolic auxotrophy.

The selection of the loss of URA3 gene containing plasmids was performed using 5-fluoroortic acid (FOA)-plates. On FOA-plates only uracil auxotroph cells can survive because the URA3 gene product converts 5-fluorotic acid into the toxic compound 5-fluouracil.

FOA-plates
$\begin{array}{ll} & 0.2 \%(\mathrm{w} / \mathrm{v}) \text { Yeast drop out mix } \\ & 0.17 \%(\mathrm{w} / \mathrm{v}) \text { Yeast nitrogen base } \\ & 0.5 \%(\mathrm{w} / \mathrm{v}) \text { Ammonium sulfate } \\ & 0.1 \%(\mathrm{w} / \mathrm{v}) \mathrm{FOA} \\ & 2.0 \%(\mathrm{w} / \mathrm{v}) \text { Glucose } \\ & 1.8 \%(\mathrm{w} / \mathrm{v}) \text { Agar }\end{array}$

If not stated otherwise, yeast cells were grown on plates with the appropriate composition at $25{ }^{\circ} \mathrm{C}$ and plates were stored at $4{ }^{\circ} \mathrm{C}$. Liquid cultures were inoculated with single colonies from plates or from liquid pre-cultures and grown with agitation at $25{ }^{\circ} \mathrm{C}$ until logarithmic growth phase $\left(1-3 \times 10^{7}\right.$ cells $\left./ \mathrm{ml}\right)$. Cells were counted using a hemocytometer. Temperature sensitive strains and the corresponding wild type were cultivated in a water bath for the indicated time at $37^{\circ} \mathrm{C}$. Cold sensitive strains and the corresponding wild type were quickly cooled down to $16{ }^{\circ} \mathrm{C}$ in a water bath and subsequently cultivated at $16{ }^{\circ} \mathrm{C}$ in an incubator. 
Liquid cultures were harvested by centrifugation at $4000 \mathrm{~g}$ for $1 \mathrm{~min}$, washed once with sterile water and harvested again. Afterwards, cells were either directly used or frozen in liquid nitrogen and stored at $-20{ }^{\circ} \mathrm{C}$.

For the generation of new yeast strains, two haploid strains of opposite mating types were crossed on selective media plates. Super-SPO medium was inoculated with the resulting diploids and cultivated at least three days up to one week. The low nutrient amount forces the diploid yeast cells to undergo meiosis. Thus, the diploid cells build four haploid cells (tetrad) surrounded by an ascus wall. Zymolyase digestion enables the dissection of tetrads with a tetrad microscope (Eclipse E400, Nikon). The cells were grown on full medium (YPD)-plates for two days. The resulting single cell colonies were tested for the desired genotype, by analytic PCRs and further analyses, e.g. western blot analysis and GFP-microscopy.

Super-SPO medium $\quad$ Solution 1: $\quad 0.5 \%(\mathrm{w} / \mathrm{v})$ Yeast extract

$306 \mathrm{mM}$ Potassium acetate

Solution 2: $5 \mathrm{mM}$ Glucose

$5.9 \mathrm{mM}$ Threonine

$1.2 \mathrm{mM}$ Phenylalanine

$0.7 \mathrm{mM}$ Uracil

$0.4 \mathrm{mM}$ Adenine and Tyrosine

$0.3 \mathrm{mM}$ Leucine and Methionine

$0.2 \mathrm{mM}$ Histidine, Lysine, Tryptophan and Arginine

Solution 1 was autoclaved and mixed 1:1 with sterile filtered solution 2.

The mating type of haploid cells was determined by replica plating the cells on two plates with a lawn of a tester strain with defined mating type (MATa or MATa, respectively) (Sprague, 1991). After three days of incubation, the plates were plated onto B-plates, which specifically select for diploid cells. The tester strains are isoleucine and valine auxotroph and the strain of interest has different auxotrophies, too. Therefore, only diplod cells, which can compensate the auxotrophies of both strains can grow on B-plates. Thus, cells which could grow on the B-plates carry the opposing mating type of the used tester strain. 


$$
\begin{array}{ll} 
& 0.17 \%(w / v) \text { Yeast nitrogen base } \\
& 0.5 \%(w / v) \text { Ammonium sulfate } \\
& 2.0 \%(w / v) \text { Glucose } \\
& 3.0 \%(w / v) \text { Agar }
\end{array}
$$

\subsubsection{Tranformation of $S$. cerevisiae}

The transformation of $S$. cerevisiae cells was performed via the lithium acetate method modified from (Gietz et al., 1992). Cells were grown to log phase, harvested, washed twice with TE/lithium acetate buffer and resuspended, resulting in a cell density of $10^{9}$ cells $/ \mathrm{ml}$. $50 \mu \mathrm{l}$ of the cell suspension was supplemented with $50 \mu \mathrm{g}$ boiled $\left(5 \mathrm{~min} 95^{\circ} \mathrm{C}\right.$, followed by 2 min on ice) Salmon sperm-carrier (ss)DNA, 500-1000 ng plasmid DNA and $300 \mu 1$ PEG/TE/lithium acetate buffer. The mixture was incubated at $25{ }^{\circ} \mathrm{C}$ with agitation followed by a heat shock at $42{ }^{\circ} \mathrm{C}$ for $15 \mathrm{~min}$. Cells were pelleted, washed twice with sterile water and plated out on the corresponding selective medium plates. Plates were incubated at $25{ }^{\circ} \mathrm{C}$ for 2 4 days and single colonies were restreaked on selective media plates.

TE/Lithium acetate $\mathrm{pH} 7.5 \quad 100 \mathrm{mM}$ Lithium acetate

$10 \mathrm{mM}$ Tris/HCl

$1 \mathrm{mM}$ EDTA

PEG/TE/Lithium acetate $\mathrm{pH} 7.5 \quad 40 \%$ PEG 4000

$100 \mathrm{mM}$ Lithium acetate

$10 \mathrm{mM}$ Tris/HCl

1 mM EDTA

\subsection{Cloning, DNA purification and DNA analysis}

\subsubsection{Isolation of chromosomal DNA from yeast}

Genomic DNA (gDNA) was extracted from yeast cells using the phenol/chloroform method, as essentially described before (Rose et al., 1991).

Detergent Lysis buffer pH $8.0 \quad 100 \mathrm{mM} \mathrm{NaCl}$

$10 \mathrm{mM}$ Tris/HCl

1 mM EDTA

$2 \%(\mathrm{v} / \mathrm{v})$ Triton X-100

$1 \%(\mathrm{w} / \mathrm{v}) \mathrm{SDS}$ 
TE-buffer $\mathrm{pH} 8.0$

$10 \mathrm{mM}$ Tris/HCl

1 mM EDTA

Yeast cells were grown until saturation of a $10 \mathrm{ml}$ liquid culture. Cells were harvested and washed once with sterile water. Afterwards, the cells were resuspended in $200 \mu 1$ detergent lysis buffer and $200 \mu 1$ phenol/chloroform/isoamylalcohol (P/C/I) (25:24:1) and $200 \mu 1$ glass beads were added to the suspension. Cells were disrupted by using the FastPrep24 machine for three times at $6 \mathrm{~m} / \mathrm{s}$ for $20 \mathrm{~s}$. The aqueous phase was separated from the organic phase via centrifugation at 21,000 $\mathrm{g}$ for $5 \mathrm{~min}$ and washed twice with $200 \mu \mathrm{P} / \mathrm{C} / \mathrm{I}$ and once with $200 \mu \mathrm{l}$ C/I. The DNA was precipitated by the addition of $1 / 10$ volume $3 \mathrm{M}$ sodium acetate and three times volume $100 \%$ ethanol and subsequent centrifugation at $21,000 \mathrm{~g}$ for $30 \mathrm{~min}$ at $4{ }^{\circ} \mathrm{C}$. The pellet was washed twice with $70 \%$ ethanol and was dried on ice. The dry pellet was resuspended in deionized, nuclease free water and stored at $-20^{\circ} \mathrm{C}$.

\subsubsection{Isolation of plasmid DNA from $E$. coli}

Plasmid DNA from E. coli was extracted using the Nucleo-Spin Plasmid-Kit (MachereyNagel) for low amounts ( $5 \mathrm{ml}$ liquid culture) and the Nucleo-bond Xtra Midi-Kit (MachereyNagel) for high amounts ( $200 \mathrm{ml}$ liquid culture). The extraction was performed strictly regarding the manufacturer's protocols. The purified plasmid-DNA was eluted in deionized, nuclease free water and stored at $-20{ }^{\circ} \mathrm{C}$.

\subsubsection{Polymerase chain reaction}

The polymerase chain reaction (PCR) is a method to amplify DNA from a template (gDNA or plasmid DNA) (Mullis et al., 1986). In this work, PCR was used for analytical purposes using the DreamTaq-polymerase (Thermo Scientific) or for subsequent cloning using the proof reading polymerase Phusion High-Fidelity (Thermo Scientific) or Q5 (NEB). Each enzymatic reaction needs different conditions and a target specific primer pair. The conditions were chosen as described by the manufacturer's protocols. Exemplary, the standard reaction for the DreamTaq polymerase is shown.

$50 \mu 1$ DreamTaq PCR reaction:

$\begin{array}{ll}5 \mu \mathrm{l} & \text { DreamTaq buffer }(10 \mathrm{x}) \\ 1 \mu \mathrm{l} & \text { dNTPs }(10 \mathrm{mM}) \\ 1 \mu \mathrm{l} & \text { Forward primer }(100 \mathrm{mM}) \\ 1 \mu \mathrm{l} & \text { Reverse primer }(100 \mathrm{mM}) \\ 0.5 \mu \mathrm{g} & \text { Template DNA } \\ 1.25 \mathrm{U} & \text { DreamTaq polymerase } \\ 37 \mu \mathrm{l} & \mathrm{H}_{2} \mathrm{O}\end{array}$

PCR program:

$95^{\circ} \mathrm{C} \quad 3 \mathrm{~min}$

$95^{\circ} \mathrm{C} \quad 30 \mathrm{~s}$

AT ${ }^{\circ} \mathrm{C} \quad 30 \mathrm{~s}$

$72{ }^{\circ} \mathrm{C} \quad 1 \mathrm{~min} / \mathrm{kb}$

$72{ }^{\circ} \mathrm{C} \quad 10 \mathrm{~min}$
Initial denaturation

Denaturation

Annealing

Extension

Final extension
34 cycles 
The annealing temperature (AT) was dependent on the composition of the primer sequences.

\subsubsection{Agarose gel electrophoresis and DNA extraction}

The analysis of PCR products was performed by agarose gel electrophoresis. Agarose gels ( $1 \%(\mathrm{w} / \mathrm{v})$ in TAE buffer) with $0.5 \mu \mathrm{g} / \mathrm{ml}$ ethidium bromide were used. Samples were loaded on the gel with a 6x DNA loading dye. The size of the DNA was displayed by a DNA marker with the appropriate DNA range. Afterwards, the DNA was visualized and pictures were taken with the INTAS UV-System (INTAS). Products, which were used for further cloning were purified with the peqGold Gel Exraction Kit (Peqlab) using the manufacturer's protocol.

TAE buffer $\mathrm{pH} 8.5$

6x DNA loading dye
$40 \mathrm{mM}$ Tris acetate

$$
1 \text { mM EDTA }
$$

$10 \mathrm{mM}$ Tris $\mathrm{pH} 7.5$

$60 \%(\mathrm{v} / \mathrm{v})$ Glycerol

$0.03 \%(w / v)$ Bromophenol blue

$0.03 \%(\mathrm{w} / \mathrm{v})$ Xylene cyanol

\subsubsection{Digestion with restriction enzymes}

Enzymatic digestion of DNA was performed for cloning approaches or for analytical purposes. The reaction was performed under enzyme specific conditions as specified by the manufacturer. To avoid religation, the 5'-phosphate of linearized DNA was dephosphorylated by the FastAP Thermosensitive Alkaline Phosphatase ( $1 \mathrm{U}$ for $10 \mathrm{~min}$ at $37^{\circ} \mathrm{C}, 80^{\circ} \mathrm{C} 10 \mathrm{~min}$ ) (Thermo Fisher Scientific).

$20 \mu 1$ Restriction digest reaction:

$2 \mu \mathrm{l} \quad$ Enzyme specific buffer (10x)

$1 \mu \mathrm{R} \quad$ Restriction enzyme

1-5 $\mu \mathrm{l} \quad$ Plasmid DNA $(1 \mu \mathrm{g} / \mu \mathrm{l})$

12-16 $\mu \mathrm{l} \quad \mathrm{H}_{2} \mathrm{O}$

\subsubsection{Gibson Assembly}

Gibson Assembly (GA) was used to create new plasmids (Gibson, 2011; Gibson et al., 2009). GA allows the ligation of several linear DNA fragments with complementary overhangs. These fragments can be obtained by PCR with 5'-elongated primers and by enzymatic plasmid digestion with endonucleases, which produce sticky ends. GA is based on three enzymatic reactions: (i) T5-exonuclease (NEB) specific digestion of 5'-ends creates 3'- 
overhangs, which allows the annealing of complementary strands. (ii) The Phusion or Q5 DNA-polymerase (NEB) extends the overhangs and closes gaps. (iii) The annealed and filled up strands are ligated by Taq-DNA ligase (NEB).

For one GA reaction $(20 \mu \mathrm{l}) 10 \mu \mathrm{l}$ GA mastermix, 100 ng linearized plasmid DNA and 2-3 fold excess of the insert diluted in deionized, nuclease-free water were used. The reaction was incubated at $50{ }^{\circ} \mathrm{C}$ for $1 \mathrm{~h}$. Afterwards, competent $\mathrm{DH} 5 \alpha$ E. coli were transformed with the reaction mix for plasmid amplification (see 3.9.2 and 3.9.7).

2x GA master mix

$$
\begin{aligned}
& 5 \% \text { (v/v) PEG } 8000 \\
& 100 \mathrm{mM} \text { Tris/HCl pH } 7.5 \\
& 10 \mathrm{mM} \mathrm{MgCl}_{2} \\
& 10 \mathrm{mM} \text { DTT } \\
& 200 \mu \mathrm{M} \text { dNTPs } \\
& 1 \mathrm{mM} \text { NAD } \\
& 0.04 \mathrm{U} \text { T5 exonuclease } \\
& 0.25 \mathrm{U} \text { Phusion DNA polymerase } \\
& 40 \mathrm{U} \text { Taq DNA ligase }
\end{aligned}
$$

\subsubsection{Transformation of $E$. coli}

Transformation of E. coli cells was performed as described before (Inoue et al., 1990). $100 \mu 1$ competent cells were thawed on ice and mixed with up to $10 \mu 1$ GA reaction mix or with 100$1000 \mathrm{ng}$ plasmid DNA and incubated for $30 \mathrm{~min}$ on ice. Afterwards, the cells were heat shocked at $42{ }^{\circ} \mathrm{C}$ for $2 \mathrm{~min}$, pelleted and resuspended in LB-Medium for $1 \mathrm{~h}$ recovery at $25^{\circ} \mathrm{C}$. After recovery, the cells were plated out on LB-plates with the appropriate antibiotic for selection of transformed cells and cultivated at $37{ }^{\circ} \mathrm{C}$.

\subsubsection{Sanger-Sequencing}

Sanger-Sequencing of plasmid DNA was performed by LGC Genomics, Berlin, Germany. Therefore, 200-500 ng of DNA were mixed with 20 pmol of a sequence specific primer and deionized, nuclease free water to a total volume of $14 \mu \mathrm{l}$. 


\subsection{Molecular biological methods}

\subsubsection{RNA extraction from yeast cells and reaction mixtures}

RNA from whole cell lysates and cytoplasmic fractionation experiments were isolated using the NucleoSpin RNA kit (Macherey-Nagel) following the manufacturer's protocol.

RNA from RNA co-immunoprecipitations was purified with Trizol ${ }^{\circledR}\left(\right.$ Ambion ${ }^{\circledR}$ RNA by Life technologies). The beads from the RNA co-immunoprecipitation experiments were resuspended in $1 \mathrm{ml} \mathrm{Trizol}{ }^{\circledR}$ and incubated with agitation at $65^{\circ} \mathrm{C}$ for $10 \mathrm{~min}$. Afterwards, $200 \mu 1$ chloroform were added, the mixture was shaked and subsequently centrifuged at room temperature at $16,000 \mathrm{~g}$ for $15 \mathrm{~min}$. The upper phase (liquid phase) was mixed with $500 \mu \mathrm{l}$ isopropanol and $1 \mu \mathrm{l}$ GlycoBlue (Ambion) and RNA was precipitated over night at $-20^{\circ} \mathrm{C}$. The next day, the mixture was centrifuged at $21,000 \mathrm{~g}$ for $30 \mathrm{~min}$ at $4{ }^{\circ} \mathrm{C}$. The supernatant was discarded and the pellet was washed twice with ice cold $70 \%$ ethanol (diluted in DEPCtreated water). Finally, the RNA was dried at $65^{\circ} \mathrm{C}$ for $10 \mathrm{~min}$ and resuspended in DEPCtreated water. The RNA concentration was measured using the NanoDrop2000 Spectrophometer (PeqLab, Thermo Fisher Scientific) and stored at $-80{ }^{\circ} \mathrm{C}$.

\subsubsection{Reverse transcription and Quantitative Realtime-PCR}

Quantitative Realtime-PCR (qRT-PCR) is a method to measure the amount of specific RNAs in samples (Pfaffl, 2004). The method is based on specific dyes like SYBR-Green, which can fluoresce when interacting with DNA. During each cycle of the PCR with target specific primers the amount of DNA increases and with it the corresponding fluorescence, which can be measured. From the change in fluorescence, one can determine the relative amount of RNA in the different samples.

Equal amounts of isolated RNA of all samples were taken (50-1000 ng RNA) for the reverse transcription of RNA into complementary (c)DNA via the Maxima Reverse Transcriptase (Thermo Scientific) following the manufacturer's protocol. In this study the cDNA synthesis was carried out with the use of Random Hexamer Primers (Thermo Scientific). As negative control for unspecific DNA contamination, a second set of samples was treated corresponding to the protocol with the exception of the addition of a reverse transcriptase. Finally, the cDNA reaction mixture was diluted 1:20 in DEPC-treated water for use in qRT-PCR. In this study all qRT-PCRs were performed with the CFX Connect Real-Time System (BioRad). 


\subsubsection{Synthesis of digoxigenin labeled RNA probes}

Digoxigenin (DIG)-labeled probes were created in vitro by the use of the T7-polymerase (Thermo Scientific) and the DIG RNA labeling Mix (Roche). The size of the DIG-probes varied between 100 and 500 nucleotides. At first, the target region was amplified from gDNA (see 3.9.1) via PCR (DreamTaq polymerase, see 3.9.3) with one primer containing a T7promotor sequence (Table 11 and Table 12) and purified (see 3.9.4). Then, from this template the in vitro transcription was performed with DIG-labeled UTP together with all four natural RNA-NTPs. The DIG-labeled UTP is integrated at a ratio of every 20-25 nucleotides. The transcription was performed as described by the manufacturer's protocol.

The reaction was stopped by the addition of $1 \mu 10.5 \mathrm{M}$ EDTA. LiCl (final $200 \mathrm{mM}$ ), glycogen (final $80 \mathrm{ng} / \mu \mathrm{l}$ ) and ethanol (final $75 \%(\mathrm{v} / \mathrm{v})$ ) were added to the reaction mix and the DIG-RNA was precipitated overnight at $-20{ }^{\circ} \mathrm{C}$. The next day, the DIG-RNA was centrifuged at 21,000 g for $30 \mathrm{~min}$, washed twice with $70 \%(\mathrm{v} / \mathrm{v})$ ethanol and air dried. The DIG-RNA was resuspended in $60 \mu 1$ HybMix, $20 \mu 1$ deionized formamide and $20 \mu 10.5 x$ TE $\mathrm{pH}$ 7.5. The probe was stored at $-20{ }^{\circ} \mathrm{C}$.

20x SSC pH 7.0

TE buffer $\mathrm{pH} 7.5$

HybMix

50x Denharts solution

\section{$3 \mathrm{M} \mathrm{NaCl}$}

$300 \mathrm{mM}$ Sodium citrate

$10 \mathrm{mM}$ Tris/HCl

1 mM EDTA

$50 \%(\mathrm{v} / \mathrm{v})$ deionized formamide

$5 x$ SSC

$500 \mu \mathrm{M}$ EDTA

$0.1 \%$ (v/v) TWEEN-20

1x Denharts solution

$0.1 \mathrm{mg} / \mathrm{ml}$ Heparin

$1 \%(w / v)$ Ficoll

$1 \%$ (w/v) Polyvinylpyrrolidone

$1 \%(\mathrm{w} / \mathrm{v})$ Bovine serum albumin 
Table 12: Primers used for DIG-labeled probes

\begin{tabular}{l|l}
\hline Target & Primers \\
\hline U1 & HK1608 and HK1609 \\
\hline U2 & HK1723 and HK1724 \\
\hline U6 & HK1404 and HK1492 \\
\hline
\end{tabular}

\subsubsection{Nucleo-cytoplasmic fractionation}

The fractionation was performed with modifications as described earlier (Sklenar and Parthun, 2004). Liquid cell cultures $(200 \mathrm{ml})$ were grown to mid log-phase, harvested and the cells were washed once in YPD/1 M sorbitol/2 mM DTT and resuspended in YPD/1 M sorbitol/1 mM DTT. For digestion of the cell wall, $100 \mu \mathrm{g}$ zymolyase was added and the mixture was incubated for 20-60 min at room temperature until at least $80 \%$ of the cells were spheroblasted. Afterwards, cells were diluted in $50 \mathrm{ml}$ YPD/1 M sorbitol for a $30 \mathrm{~min}$ recovery step at room temperature. Then, cells were shifted to restrictive temperature for the indicated time. Cells were harvested at low speed centrifugation (900 g, $5 \mathrm{~min}$ ) and resuspended in $500 \mu \mathrm{l}$ Ficoll buffer. Cell lysis was obtained by the addition of $1 \mathrm{ml}$ lysis buffer and vortexing. The fractionation was achieved by centrifugation at 1,500 $\mathrm{g}$ for $15 \mathrm{~min}$. The supernatant was taken as cytoplasmic fraction. The purity of the cytoplasmic fraction was controlled via western blot analysis (see 3.13.6) with the cytoplasmic protein Zwf1 and the nucleolar protein Nop1.

\subsection{Microscopy}

Microscopic studies were performed with the Leica DMI6000B fluorescence microscope. Pictures were taken with the Leica DFC360 FX camera using the LAS AF1.6.2 software.

\subsubsection{Fluorescence in situ hybridization}

The fluorescence in situ hybridization (FISH) experiments with DIG-labeled probes were performed as described in (Hackmann et al., 2014).

Cy3-labled DNA-oligos (Sigma Aldrich) were used to specifically stain target RNAs in the cytoplasm. DNA-oligos used in this study were 50 nucleotides long, reverse complementary to the target RNA and labeled with $\mathrm{Cy} 3$ at the 5' and/or the 3'end (Table 11). For FISH experiments with Cy3-labeled DNA-oligos, steps up to pre-hybridization were the same as described in (Hackmann et al., 2014). Then, the Cy3-labled oligos were diluted 1:200 in HybMix (see 3.10.3) and the cells were hybridized over night at $37^{\circ} \mathrm{C}$ in a humidified chamber. The next day, cells were washed once with 2x SSC, once with 1x SSC and finally twice with $0.5 \mathrm{x} \mathrm{SSC}$ at $37{ }^{\circ} \mathrm{C}$ and at room temperature, respectively. Afterwards, the staining 
of DNA was performed with Hoechst (diluted 1:10,000 in PBS) for 2 min at room temperature followed by three washing steps, each for $5 \mathrm{~min}$ at room temperature (once with PBS containing $0.1 \%$ TWEEN-20 and twice with PBS).

PBS

$$
\begin{aligned}
& 137 \mathrm{mM} \mathrm{NaCl} \\
& 3 \mathrm{mM} \mathrm{KCl} \\
& 10 \mathrm{mM} \mathrm{Na}_{2} \mathrm{HPO}_{4} \\
& 2 \mathrm{mM} \mathrm{KH}_{2} \mathrm{PO}_{4}
\end{aligned}
$$

\subsubsection{GFP microscopy}

GFP microscopy was performed as described earlier in (Windgassen et al., 2004) combined with DNA staining described in 3.11.1.

\subsection{In vitro binding studies}

For the determination of the RNA binding capacity of Cse1 via in vitro binding assays, recombinant GST-Cse1 was expressed in E. coli and purified via affinity purification.

\subsubsection{Expression and purification of recombinant GST-Cse1}

To purify recombinant GST-Cse1, E. coli BL21* cells carrying pGEX-6P-1 AmpR Ptac:GSTCse1 (pHK1476) were grown in an $5 \mathrm{ml}$ overnight culture in LB-medium containing ampicilin $(100 \mu \mathrm{g} / \mathrm{ml})$. Afterwards, a 21 culuture of auto-inducing medium containing ampicilin $(100 \mu \mathrm{g} / \mathrm{ml})$ was inoculated with the overnight pre-culture and incubated for $72 \mathrm{~h}$ at $16^{\circ} \mathrm{C}$. Cells were harvested, washed once in binding buffer, resuspended in $2 \mathrm{ml}$ binding buffer per mg cells and treated with benzonase and lysozyme for $10 \mathrm{~min}$ at $4{ }^{\circ} \mathrm{C}$. Then, cells were disrupted with the LM10 Microfluidizer (Microfluidics). The lysate was centrifuged at $30,000 \mathrm{~g}$ for $30 \mathrm{~min}$ in the Sorvall WX Ultra Series Centrifuge (Thermo Scientific) using the Ti50 Rotor (Beckmann). The cleared supernatant was loaded on two $1 \mathrm{ml}$ GSTrap ${ }^{\mathrm{TM}}$ FF (GE Healthcare) columns using the Äktaprime plus (GE Healthcare). Cse1-bound RNA was eluted from the column by $1 \mathrm{M} \mathrm{LiCl-solution}$ treatment. Finally, the protein was eluted in elution buffer. Protein containing fractions were determined via SDS-PAGE (see 3.13.4) and subsequent silver staining (see 3.13.5) as well as western blot analysis (anti-GST 1:2000, Table 6) (see 3.13.6). The identified fractions were dialyzed in 21 binding buffer over night at $4{ }^{\circ} \mathrm{C}$ including change of buffer twice. Protein was concentrated with Vivaspin 20 columns (Viva Science, Sartorius Group) and protein concentration was determined with the $\mathrm{DC}^{\mathrm{TM}}$ 
protein assay (Bio-Rad) following the manufacturer's protocols. The purified protein was stored at $-20{ }^{\circ} \mathrm{C}$.

Autoinducing medium

$$
\begin{aligned}
& \text { LB medium (see 3.8.1) } \\
& 25 \mathrm{mM} \mathrm{K}_{2} \mathrm{HPO}_{4} \\
& 25 \mathrm{mM} \mathrm{NaH}_{2} \mathrm{PO}_{4} \\
& 50 \mathrm{mM} \mathrm{NH}_{4} \mathrm{Cl} \\
& 5 \mathrm{mM} \mathrm{Na}_{2} \mathrm{SO}_{4} \\
& 1 \mathrm{mM} \mathrm{MgSO}_{4} \\
& 0.5 \%(\mathrm{v} / \mathrm{v}) \text { glycerine } \\
& 0.05 \% \text { glucose } \\
& 0.2 \% \text { lactose }
\end{aligned}
$$

Binding buffer

$20 \mathrm{mM}$ Tris- $\mathrm{HCl} \mathrm{pH} 8.0$

$50 \mathrm{mM} \mathrm{NaCl}$

$1 \mathrm{mM}$ DTT

Elution buffer

Binding buffer

$40 \mathrm{mM}$ glutathione

\subsubsection{In vitro $\mathrm{RNA}$ co-immunoprecipitations}

The binding of RNA to GST-Cse1 was examined via RNA co-immunoprecipitation experiments (RIPs) (see 3.13.2). RIPs were performed using a GST antibody (1:200, Table 6) and Protein $\mathrm{G}$ sepharose beads (Amersham Biosciences) adding $50 \mu \mathrm{g}$ recombinant GSTCse1 and $100 \mu \mathrm{g}$ total RNA. RNA was extracted using Trizol ${ }^{\circledR}$ (Ambion ${ }^{\circledR}$ RNA by Life technologies ${ }^{\mathrm{TM}}$ ) (see 3.10.1). The purified RNA was reverse transcribed with Maxima reverse transcriptase (Thermo Scientific) for subsequent qRT-PCR analyses (see 3.10.2).

\subsection{Protein and RNA biochemical methods}

\subsubsection{Preparation of yeast cell lysates}

Cells were grown to mid-log phase, treated as indicated for each experiment and harvested. Cells were washed once with sterile water and either directly used or frozen in liquid nitrogen and stored at $-20^{\circ} \mathrm{C}$. 
Lysis of yeast cells was performed with the same volume of the according buffer (see 3.13.2) the same volume of glass beads (SiLibeads type S 0.4-0.6 mm, Th. Geyer), protease inhibitor (complete EDTA-free, Roche) and for RIPs also Ribolock RNase Inhibitor (Thermo Scientific). Cells were ruptured using three times the FastPrep24 machine at $6 \mathrm{~m} / \mathrm{s}$ for $20 \mathrm{sec}$ with cooling on ice between each step for at least $1 \mathrm{~min}$. Lysates were centrifuged for $5 \mathrm{~min}$ at 4,000 $\mathrm{g}$ and 21,000 $\mathrm{g}$ for $3 \mathrm{~min}$. The cleared lysate was used for the experiments.

\subsubsection{Protein-protein and protein-RNA co-immunoprecipitations}

The experiments were essentially performed as published earlier (Zander et al. 2016). All yeast strains were grown to $\log$ phase $\left(1-3 * 10^{7}\right.$ cells $\left./ \mathrm{ml}\right)$.

For protein-protein co-immunoprecipitations and RIPs, the cells were harvested and lysed in the appropriate buffer (PBSKMT for protein-protein and RIP buffer for protein-RNA IPs). The cleared lysate was incubated for $3 \mathrm{~h}$ with agitation at $4{ }^{\circ} \mathrm{C}$ with GFP-Trap ${ }^{\circledR}$ A beads (Chromotek), which were washed three times with buffer before use. After incubation, the beads were washed six times with buffer. For RIPs the beads were split into equal amounts, for protein detection and RNA isolation, respectively. Proteins were detected by western blot analyses with the indicated antibodies (Table 6) (see 3.13.4 and 3.13.6). RNA was extracted using Trizol ${ }^{\circledR}$ (Ambion ${ }^{\circledR}$ RNA by Life technologies ${ }^{\mathrm{TM}}$ ) (see 3.10.1). The purified RNA was reverse transcribed with Maxima reverse transcriptase (Thermo Scientific) for subsequent qRT-PCR analyses (see 3.10.2).

RIP buffer $\mathrm{pH} 7.5$

$25 \mathrm{mM}$ Tris/HCl pH 7.5

$150 \mathrm{mM} \mathrm{NaCl}$

$2 \mathrm{mM} \mathrm{MgCl}{ }_{2}$

$0.2 \%$ (v/v) Triton-X-100 (freshly added)

$0.2 \mathrm{mM}$ PMSF (freshly added)

$0.5 \mathrm{mM}$ DTT (freshly added)

DNase (50 Kunitz units) (freshly added)

RiboLock (0.02 U/ml) and Protease Inhibitor

(freshly added) 
PBSKMT buffer $\mathrm{pH} 7.5$

1x PBS pH 7.5

$3 \mathrm{mM} \mathrm{KCl}$

$2.5 \mathrm{mM} \mathrm{MgCl} 2$

$0.5 \%(\mathrm{v} / \mathrm{v})$ Triton-X-100 (freshly added)

\title{
3.13.3 TMG-cap immunoprecipitation
}

For the TMG-cap specific RNA immunoprecipitation experiments, $50 \mu \mathrm{g}$ total RNA (see 3.10.1) was incubated with agitation for $1 \mathrm{~h}$ at $4{ }^{\circ} \mathrm{C}$ with Anti-2,2,7-trimethylguanosineantibody (Milipore) and Protein G sepharose beads (Amersham Biosciences) in TMG-IP buffer. The beads were washed 5 times in TMG-IP buffer. Afterwards, RNA was isolated and reverse transcribed as described in 3.10.2.

TMG-IP buffer

\author{
$25 \mathrm{mM}$ Tris/HCl pH7.5 \\ $10 \mathrm{mM} \mathrm{MgCl} 2$ \\ $0.2 \mathrm{mM}$ PMSF (freshly added) \\ 5 mM DTT (freshly added) \\ RiboLock and Protease Inhibitor (freshly added)
}

In this study, additionally a two step TMG-cap RIP was performed. Therefore, a normal RIP experiment with Prp40-GFP as described in 3.13.2 was performed with the exception that $200 \mathrm{ng}$ of the isolated RNA was used for a subsequent TMG-cap RIP. The purified RNA from this TMG-cap RIP was reverse transcribed with Maxima reverse transcriptase (Thermo Scientific) for subsequent qRT-PCR analyses (see 3.10.2).

\subsubsection{SDS-acrylamide gel electrophoresis}

Proteins from cell lysates, IPs, RIPs and protein purifications were analyzed and separated by their molecular weight via denaturing sodium dodecyl sulfate polyacrylamide gelelectrophoresis (SDS-PAGE) as described in (Garfin, 2009).

In brief, samples were mixed with 2x SDS loading buffer (Laemmli, 1970) boiled for 5 min at $95^{\circ} \mathrm{C}$ and centrifuged at $21,000 \mathrm{~g}$ for $10 \mathrm{sec}$. Samples were loaded on a bipartite gel composed of a $5 \%$ staking gel and a $10 \%$ resolving gel. As size standard the Page Ruler Prestained Protein Ladder (Thermo Scientific) was used. Electrophoresis was performed at $7 \mathrm{~mA}$ over night or at $25 \mathrm{~mA}$ for $1 \mathrm{~h}$ followed by $40 \mathrm{~mA}$. 
Staking gel (5\%)

$16.7 \%$ (v/v) Rotiphorese Gel 30 acrylamide mix

125 mM Tris/HCl pH 6.8

$0.1 \%(\mathrm{w} / \mathrm{v}) \mathrm{SDS}$

$0.1 \%(\mathrm{w} / \mathrm{v})$ Ammoniumpersulfate (APS)

$0.1 \%$ (v/v) Tetramethylethylendiamin (TEMED)

Resolving gel (10\%)

$33.3 \%$ (v/v) Rotiphorese Gel 30 acrylamide mix

375 mM Tris/HCl pH 8.0

$0.1 \%(\mathrm{w} / \mathrm{v}) \mathrm{SDS}$

$0.1 \%(\mathrm{w} / \mathrm{v})$ APS

$0.1 \%(\mathrm{v} / \mathrm{v})$ TEMED

Running buffer

$25 \mathrm{mM}$ Tris

192 mM Glycine

$0.1 \%(\mathrm{w} / \mathrm{v}) \mathrm{SDS}$

2x SDS-loading buffer

125 mM Tris/HCl pH 6.8

$25 \%$ (v/v) Glycerol

$2 \%(\mathrm{w} / \mathrm{v}) \mathrm{SDS}$

$5 \%$ (v/v) $\beta$-Mercaptoethanol

Bromophenol blue

\subsubsection{Silver staining}

For the staining of protein in SDS-polyacrylamide gels the PageSilver Silver staining Kit (Fermentas) was used. The procedure was exactly performed as described in the manufacturer's protocol.

\subsubsection{Western blot analysis}

For the analysis of specific proteins in SDS-polyacrylamide gels, proteins can be transferred from the SDS-polyacrylamide gel to a nitrocellulose membrane via Western Blot (AlegriaSchaffer, 2014; Towbin et al., 1979).

The proteins were transferred onto Amersham Protran $0.45 \mu \mathrm{m}$ nitrocellulose membrane (GE Healthcare Life Sciences) using a semi-dry blotting system (PeqLab). The transfer was 
conducted at $1.5 \mathrm{~mA} / \mathrm{cm}^{2}$ for $1.5-2 \mathrm{~h}$ depending on the size of proteins. After transfer, free available protein binding sites were blocked by incubating the membrane with $5 \%(\mathrm{w} / \mathrm{v})$ Difco skim milk (BD) in TBST. After, primary antibodies in $0.5 \%(\mathrm{w} / \mathrm{v})$ milk powder in TBST were applied to the membrane at $4{ }^{\circ} \mathrm{C}$ over night. The membrane was washed three times in TBST and then, the horseradish peroxidase conjugated secondary antibody was incubated with the membrane for $2 \mathrm{~h}$ at room temperature followed by three washing steps in TBST again. Finally, the detection was performed with the WesternBright Chemoluminescence Substrate Quantum (Biozym) and the Fusion SL chemoluminescence detection system (Peqlab).

Blotting buffer

$25 \mathrm{mM}$ Tris base

192 mM Glycine

$20 \%(\mathrm{v} / \mathrm{v})$ Methanol (freshly added)

TBST pH 7.4

$50 \mathrm{mM}$ Tris $\mathrm{pH} 7.4$

$150 \mathrm{mM} \mathrm{NaCl}$

$0.1 \%(\mathrm{v} / \mathrm{v})$ Tween 20

\subsection{RNA-Sequencing analysis}

For genome wide analysis of intron containing transcripts, strains were shifted to $37{ }^{\circ} \mathrm{C}$ for 1 h. RNA was isolated using the Nucleo-Spin RNA Kit (Macherey-Nagel) (see 3.13.1). The sequencing of RNA samples and the raw data processing was conducted at the Microarray and Deep-Sequencing Facility Göttingen (Transcriptome and Genome Analysis Laboratory, TAL) by Dr. Thomas Lingner and Dr. Gabriela Salinas as described earlier in (Zander et al., 2016) with the following exceptions: (i) Single read (50 bp) sequencing was conducted using a HiSeq 4000 (Illumina); (ii) Samples were demultiplexed to FASTQ files with bcl2fatsq (version 2.17); (iii) Sequences were aligned to the $S$. cerevisiae genome reference using the STAR software ((Dobin et al., 2013); version 2.5).

Intron retention analysis was performed by comparing fold changes of exonic expression between wild type and mex67-5 xpol-1 against fold changes of intronic expression. The sequencing data and abundance measurement files have been submitted to the NCBI Gene Expression Omnibus (GEO) under the accession number GSE93307. 


\subsection{Quantification and statistical analysis}

All experiments shown in this work were performed at least three times independently. Error bars represent the standard deviation with exception of Figure 17 were the standard error of the mean (SEM) is shown. $\mathrm{P}$ values were calculated using a one-tailed, two-sample unequal variance t-test. $\mathrm{P}$ values are indicated as follows: $* * * \mathrm{p}<0.001, * * \mathrm{p}<0.01,{ }^{*} \mathrm{p}<0.05$. For quantification of cells with displayed phenotypes for each strain a minimum of 100 cells in total was counted. 


\section{Results}

\subsection{Yeast snRNAs are exported to the cytoplasm by Mex67 and Xpo1}

As it has been shown earlier that Mex67 and Xpo1 are essential for the export of mRNAs and the ncRNA TLC1 to the cytoplasm (Hurt et al., 2000; Sträßer et al., 2000; Wu et al., 2014), we performed fluorescent in situ hybridisation (FISH) experiments to answer the question if the snRNAs shuttle and whether this export is also dependent on the two export receptors. The two temperature sensitive (ts) mutants mex67-5 and xpol-1 were used, as well as the double mutant mex67 xpo1-1 and rat7-1 and rat8-2. Both Rat7/Nup159 and Rat8/Dbp5 are related to the Mex67-Mtr2-dependent RNA export (Folkmann et al., 2011; Kelly and Corbett, 2009; Tieg and Krebber, 2013; Wu et al., 2014).

In wild type cells, snRNAs are located in the nucleus. We aimed to establish a FISH method that enables us to show a nuclear accumulation in the export deficient strains by using long fluorescently labeled probes. The Sm-class snRNAs were hybridized using DIG-labeled RNAs with a size of 200-450 nucleotides and U6 with a size of 100 nucleotides, respectively and detected with a FITC conjugated $\alpha$-DIG-antibody. Due to the length of the DIG-labeled RNAs we were able to visualize the nuclear accumulation of snRNAs in dot-like structures (Figure 11A).

A

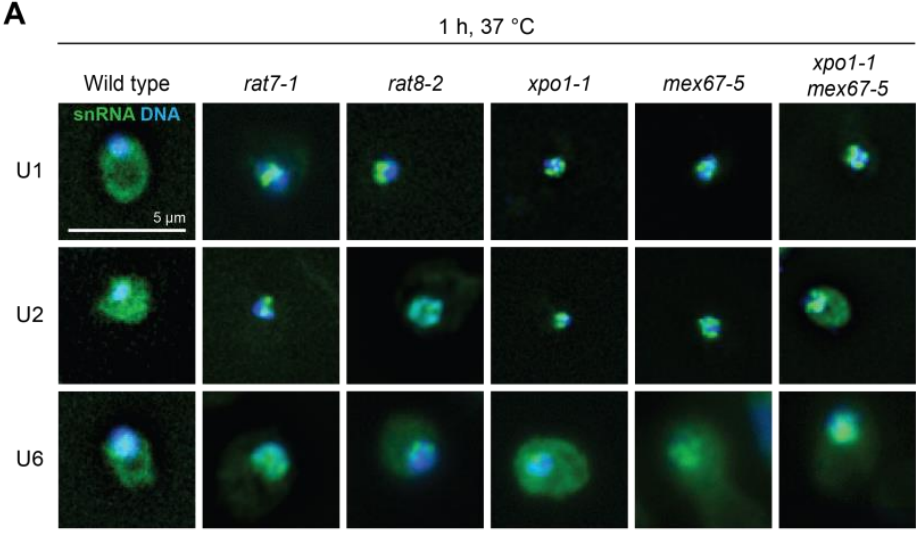

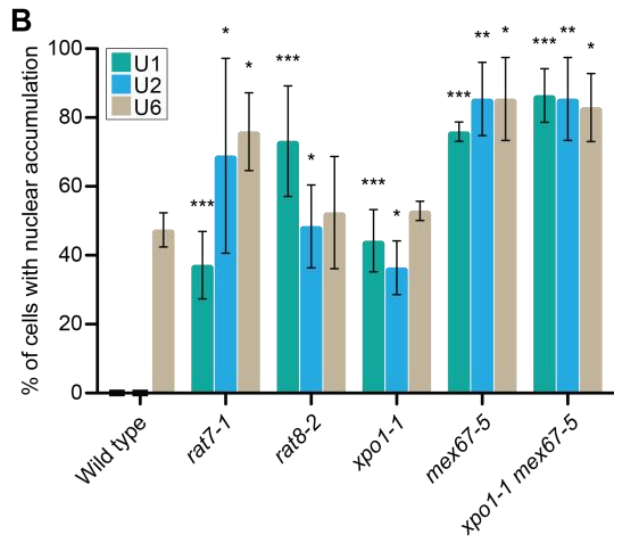

Figure 11: snRNAs show nuclear export defects in mRNA-export mutant strains. (A) Fluorescent in situ hybridization experiments reveal a dot-like nuclear accumulation of U1, U2 and U6 in the indicated strains after temperature shift for $1 \mathrm{~h}$ to $37^{\circ} \mathrm{C}$. Cells were fixed with $3.7 \%$ formaldehyde and snRNAs were hybridized with 100-400 nucleotide long, DIG-labeled RNA probes and detected via FITC conjugated $\alpha$-DIG-antibody (green). The DNA was stained with Hoechst (blue). (B) Quantification of cells with a nuclear signal shown in (A). The error bars indicate the standard deviation and $p$-values were calculated by an unpaired, one sided student's t-test $\left({ }^{*}=p<0.05\right.$, $\left.{ }^{* *}=p<0.01,{ }^{* * *}=p<0.001\right)$.

In wild type cells the signal for U1 and U2 appeared to be equally distributed throughout the cells. In contrast, rat7-1 and rat8-2 showed a significant nuclear retention of snRNAs. In rat7- 
$136 \%$ of the cells displayed a nuclear dot-like snRNA accumulation for U1 and $68 \%$ for U2. For rat8-2, the results were comparable ( $72 \%$ for U1 and $48 \%$ for U2). Mutations in XPO1 also led to a nuclear accumulation of both the snRNAs U1 (43\%) and U2 (35\%). The strongest nuclear signal was observed in mex67-5 and the double mutant mex67-5 xpo1-1.75$85 \%$ of the cells showed a nuclear accumulation. Taken together, these data indicate that the RNAP II transcribed snRNAs U1 and U2 leave the nucleus via the two export receptors Mex67 and Xpo1.

For the RNAP III transcript U6 so far no shuttling has been reported (Matera and Wang, 2014; Sloan et al., 2016). For U6 we detected a slight signal in the nucleus of wild type cells (47\%). However, our data clearly indicate that U6 is exported to the cytoplasm via Mex67 (Figure 11A, B). Like U1 and U2, U6 accumulates in $82 \%$ of the nuclei of mex67-5 cells, which is a significant change compared to $47 \%$ in wild type cells (Figure 11B). Interestingly, for Xpo1 we detected no nuclear accumulation for xpo1-1 compared to wild type.

\subsection{Mex67 and Xpo1 physically interact with the snRNAs in vivo and in vitro}

For further support of the results, we performed RNA co-immunoprecipitation (RIP) experiments with GFP-tagged Mex67 and Xpo1 in vivo. In fact, we could detect an interaction for both proteins, Xpo1 and Mex67, with the snRNAs (Figure 12A). In this experiment, the binding of Mex67 to the snRNAs was stronger than the binding of Xpo1. Compared to the no tag control, Mex67 binding to U1 was enriched 19 times and to U6 at least six times. As a negative control for unspecific interactions between GFP and the RNA we used a strain transformed with a plasmid encoding for GFP-NLS-NES. These results may also reflect the minor export defect for xpo1-1 shown in Figure 11. Interestingly, we could not detect any interaction between Xpo1 and U6, supporting the hypothesis that Xpo1 is not needed for the export of U6. The correct pulldown of the proteins and the purity of the analyzed probes were verified by western blot analyses (Figure $12 \mathrm{~B}$ ).

Mex67 was reported to directly bind to different kinds of RNAs like the 5S rRNA, mRNA and heat stress induced mRNA (Yao et al., 2007; Zander et al., 2016). Mex67 contains an RNA binding loop, which is essential for this RNA binding. Two mutant forms of recombinant Mex67 that were unable to bind mRNA in vitro were used for in vitro binding studies (Zander et al., 2016). The co-immunoprecipitated RNA of these experiments (performed by Dr. Lysann Bender) was used in this study to examine the capability of Mex67 to directly bind snRNAs in vitro. Indeed, an interaction was observed and a significant 
reduction of this interaction was shown for the mutated proteins mex67 KR>AA and mex67 $\Delta$ loop (Figure 12B).

A

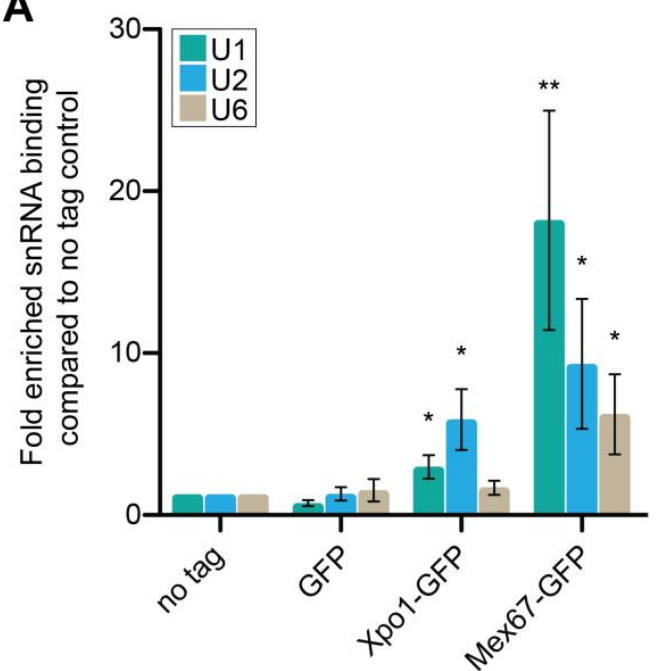

B

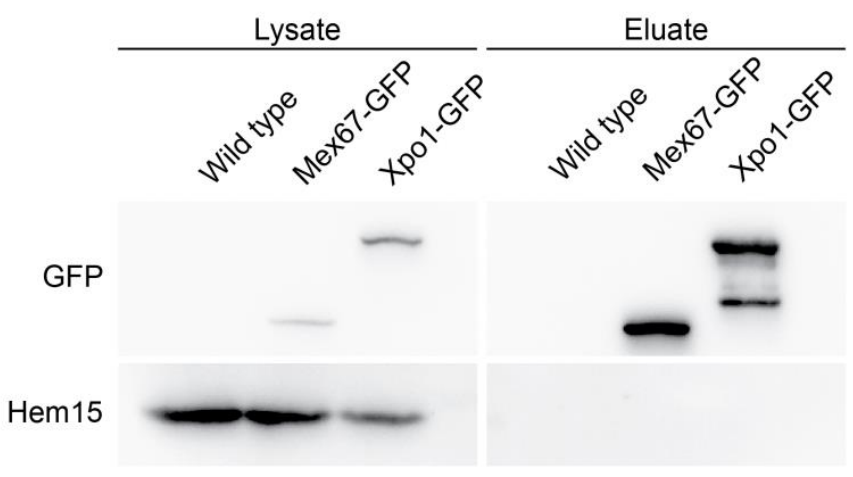

Figure 12: Mex67 and Xpo1 physically interact with the snRNAs in vivo. (A) RIP-experiments with GFP tagged Mex67 and Xpo1 reveal a physical interaction between both proteins and the snRNAs. Cells expressing GFP served as a negative control. Immunoprecipitated RNA was reverse transcribed into cDNA that was used for quantitative Real Time PCR (qRT-PCR) analyses. The error bars indicate the standard deviation and $p$-values were calculated by an unpaired, one sided student's t-test $\left({ }^{*}=\right.$ $p<0.05,{ }^{* *}=p<0.01,{ }^{* * *}=p<0.001$ ). (B) Western blot analysis of immunoprecipitated proteins from RIP experiments shown in (A). Zwf1 served as a control for unspecific protein binding to the GFP-trap beads. Indicated GFP-tagged proteins were detected with GFP-specific antibodies.

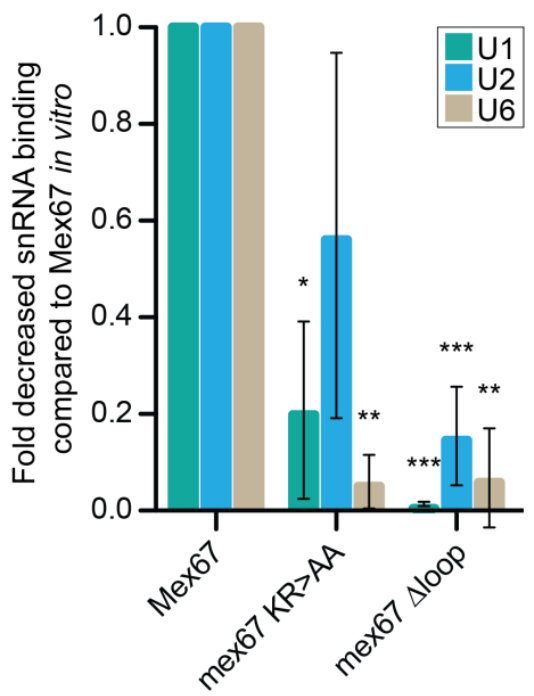

Figure 13: Mex67 physically interacts with the snRNAs in vitro. In vitro RIP experiments with recombinant Mex67, mex67 KR>AA and mex67 $\Delta$ loop kindly provided from Bender and Krebber were used to quantify the fold decreased binding of the mex67 mutants to the snRNAs. For both mutants a significant decrease was measured. The error bars indicate the standard deviation and $p$-values were calculated by an unpaired, one sided student's t-test $\left({ }^{*}=p<0.05,{ }^{* *}=p<0.01,{ }^{* *}=p<0.001\right)$. 
Taken together these data clearly suggest that snRNAs are exported in yeast via Mex67 and Xpo1. In addition, we show that U6 also shuttles to the cytoplasm, however for this export only seems to require Mex67 and not Xpo1.

\section{3 snRNAs are imported into the nucleus via Mtr10 and Cse1}

As the snRNAs are exported to the cytoplasm, we also wanted to investigate how they return back to the nucleus. In human cells, SPN binds the cytoplasmic snRNAs and the import is facilitated by importin $\beta$ (Matera and Wang, 2014). As yeast cells lack an SPN homologue, we searched for import mutants via FISH experiments and localized the snRNAs in mutants of all yeast karyopherins (Figure 14). For labeling of the snRNAs, we used a short 50 nucleotide long DNA-probe that was conjugated with $\mathrm{Cy} 3$ at its 3'- and 5'-end. These probes penetrated properly the nucleus and were able to stain the nuclear localized snRNAs. When we detected them in the transportin mutants we found strains with cytoplasmic snRNAs.
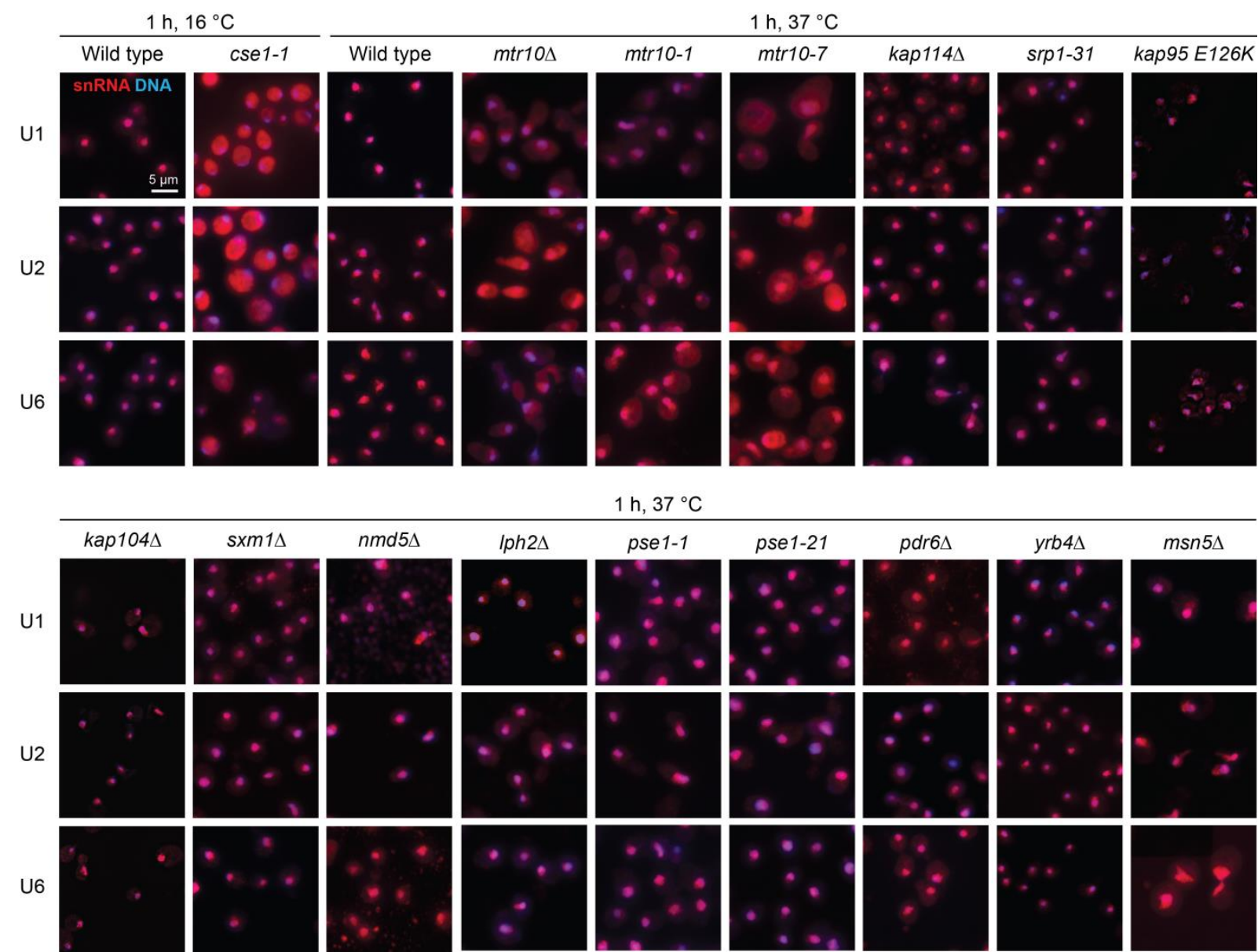

Figure 14: Analysis of import defects of snRNAs in mutants of all yeast karyopherins. Shown are fluorescent in situ hybridization experiments of $\mathrm{U} 1, \mathrm{U} 2$ and $\mathrm{U} 6$ in karyopherin mutant strains after $1 \mathrm{~h}$ temperature shift to $16^{\circ} \mathrm{C}$ or $37^{\circ} \mathrm{C}$. Cells were fixed with $3.7 \%$ formaldehyde and snRNAs were hybridized with 50 nucleotidet long, Cy3-labelled DNA probes (red). The DNA was stained with Hoechst (blue). 
While most of the mutants showed no localization defect, we could identify two karyopherins, Mtr10 and Cse1, which, if mutated, resulted in a cytoplasmic accumulation of snRNAs. Mtr10 was already described to import the RNA binding protein Npl3 and the ncRNA TLC1 to the nucleus (Gallardo et al., 2008; Senger et al., 1998). Cse1 was a surprising finding, because up to date it was only described as an export factor for importin $\alpha$ (Hood and Silver, 1998; Solsbacher et al., 1998) and proposed to be in a closed conformation in the cytoplasm, which is not able to bind a cargo (Cook et al., 2005). In addition, we could not observe any import defects for the snRNAs in importin $\beta /$ Kap95 and importin $\alpha / \operatorname{Srp} 1$ deficient strains (Figure 15A, B). This argues for a different import mechanism compared to higher eukaryotes. In numbers, around 60 to $80 \%$ of the cse $1-1$ cells accumulated the snRNAs in the cytoplasm, whereas $m$ tr $10 \Delta$ cells showed a phenotype in 30 to $70 \%$ of the counted cells (Figure 15 B).

A
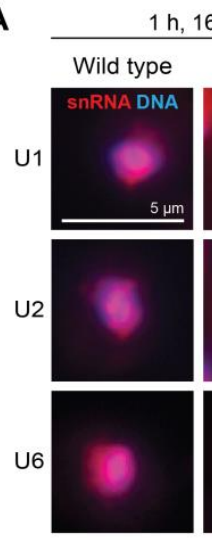

$\mathrm{h}, 16{ }^{\circ} \mathrm{C}$
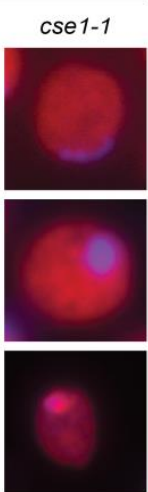
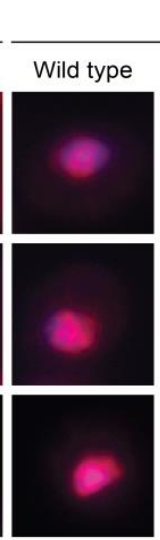

$1 \mathrm{~h}, 37^{\circ} \mathrm{C}$
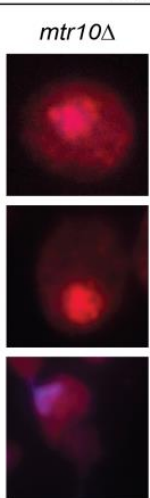

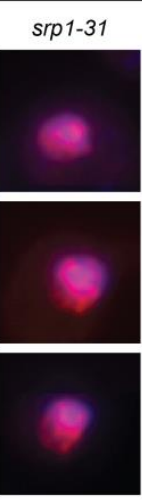

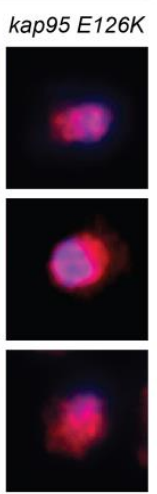

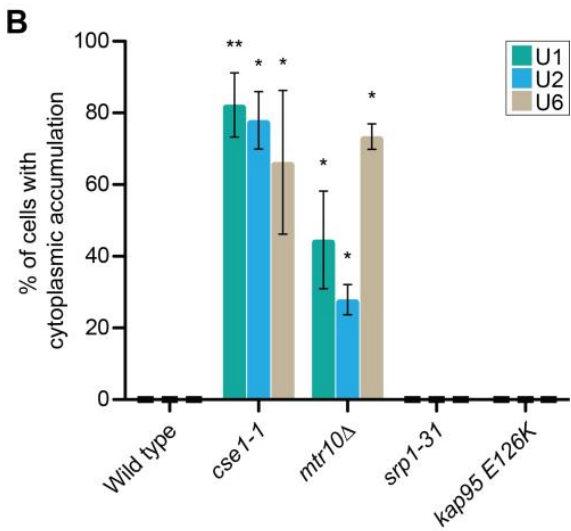

Figure 15: Cse1 and Mtr10 are responsible for the snRNA import. (A) Shown are single cell views of the indicated strains. (B) Quantification of cells with a nuclear signal shown in (A). The error bars indicate the standard deviation and $p$-values were calculated by an unpaired, one sided student's $t$ test $\left({ }^{*}=p<0.05,{ }^{* *}=p<0.01,{ }^{* * *}=p<0.001\right)$.

To confirm our initial results, we performed cytoplasmic fractionation experiments in cse 1-1 and mtr10 4 cells. After isolating the total RNA from the cytoplasmic fractions we performed a qRT-PCR and were able to measure a significant accumulation of the snRNAs in the cytoplasm compared to wild type (Figure 16A). The snRNAs were enriched between two- and four-fold. As a control for a successful cytoplasmic fractionation, we conducted western blot analyses (Figure 16B). We detected Zwf1, the glucose-6-phosphate dehydrogenase, as a cytoplasmic marker and Nop1, a nucleolar protein, as a nuclear marker. 
A

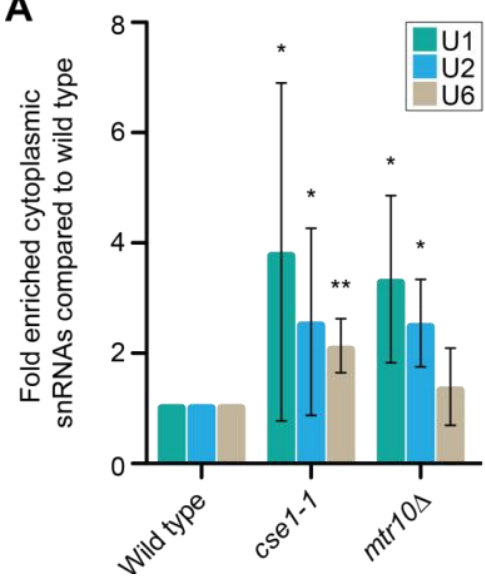

B

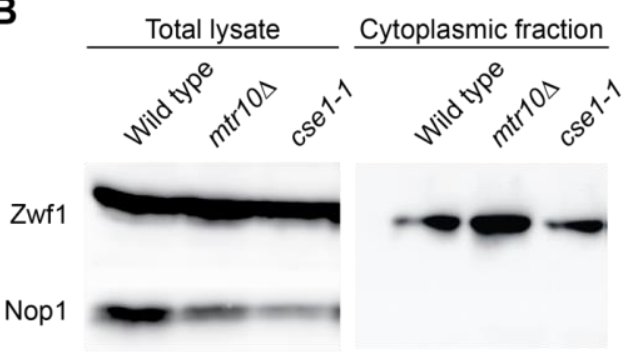

Figure 16: snRNAs are enriched in the cytoplasmic fractions of cse1-1 and mtr10 $\Delta$ cells. (A) Cytoplasmic fractionation experiments are shown. Cells were shifted to $16^{\circ} \mathrm{C}$ for cse $1-1$ or to $37^{\circ} \mathrm{C}$ for $m \operatorname{tr} 10 \Delta$ for $1 \mathrm{~h}$ prior to cell lysis and fractionation. The error bars indicate the standard deviation and $p$-values were calculated by an unpaired, one sided student's t-test $\left({ }^{*}=p<0.05,{ }^{* *}=p<0.01\right.$, $\left.{ }^{* * *}=p<0.001\right)$. (B) Western blot analysis of cytoplasmic fractionation experiments shown in (A). Total lysate and cytoplasmic fractions were analyzed by detecting the cytoplasmic marker protein Zwf1 and the nucleolar protein Nop1.

Finally, we provided evidence for the physical interaction of Cse1 and Mtr10 with the snRNAs by RIP experiments. As shown in Figure 17 we could detect a strong interaction between Cse1 and both U1 and U2. The interaction of U6 with Cse1 was quite low, indicating that a different karyopherin may be needed for U6 import. For Mtr10, we detected an enrichment for the three snRNAs U1, U2 and U6 between 5 and 2.5 fold.

A

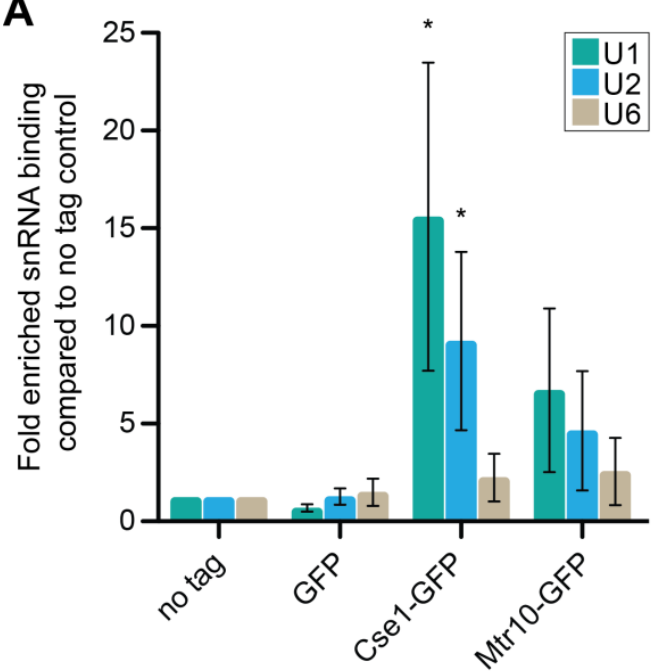

B

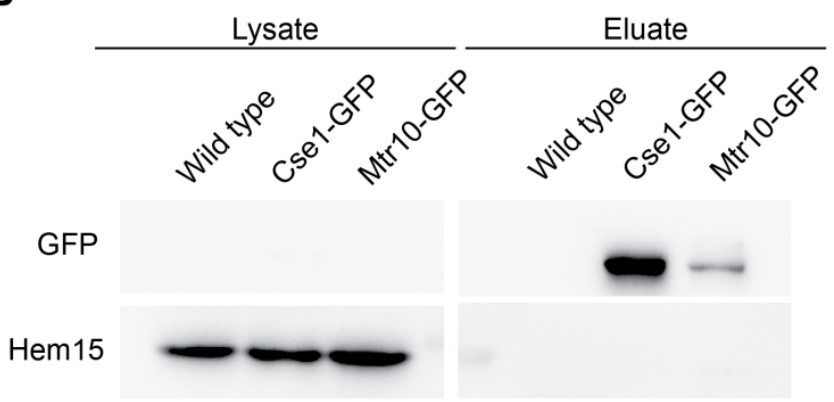

Figure 17: Cse1 and Mtr10 physically interact with the snRNAs. (A) RIP-experiments with GFP tagged karyopherins reveal a physical interaction between Cse1 and Mtr10 and the snRNAs. Cells expressing GFP alone served as a negative control. Immunoprecipitated RNA was reverse transcribed into CDNA and afterwards used for quantitative PCR ( $\mathrm{qRT}$-PCR) analyses. The error bars indicate the standard error of the mean and $p$-values were calculated by an unpaired, one sided student's $t$-test $\left({ }^{*}=p<0.05,{ }^{* *}=p<0.01,{ }^{* * *}=p<0.001\right)$. (B) Western blot analysis of immunoprecipitated proteins from RIP experiments shown in (A). Zwf1 served as a control for unspecific protein binding to the GFP-trap beads. Indicated GFP-tagged proteins were detected with GFP-specific antibodies. 
Summed up, the results provide evidence that the import of snRNAs is distinct to the one in higher eukaryotes. The two karyopherins Cse1 and Mtr10 were identified as essential importins. We could show a physical interaction between both Mtr10 and Cse1 and the snRNAs U1 and U2 as well as cytoplasmic accumulations in mutants of both karyopherins.

\subsection{Sm-ring binding assembly takes place in the cytoplasm and is essential for snRNA re-import}

An important step in the snRNA maturation is the Sm-ring assembly, which is essential for the stability of snRNAs. Mutations in the Sm-ring binding site lead to the degradation of snRNAs via Rrp6 and Xrn1 (Coy et al., 2013; Shukla and Parker, 2014). As snRNAs are supposed to be degraded by the nuclear exosome (Coy et al., 2013), as well as by the cytoplasmic decapping machinery (Dcp1-Dcp2 and Xrn1) (Shukla and Parker, 2014), we wanted to determine the place of Sm-ring assembly. Therefore, we localized SmB-GFP and SmG-GFP in mutants of the snRNAs importins Cse1 and Mtr10. To confirm the absence of a functional role in snRNA import, we also performed GFP microscopy experiments in srpl-31 and kap95 E126K.

Interestingly, we could detect a very strong mislocalization of SmB-GFP in both cse 1-1 and mtr104 (Figure 18A). Between 60 and $80 \%$ of the cse1-1 cells displayed an SmB-GFP mislovcalization and between 60 and $100 \%$ did so for mtr10د (Figure 18B). In addition, we could observe cytoplasmic foci of SmG-GFP in both mutants. This result shows that the Smring nuclear import is dependent on Cse1 and Mtr10. Mutants of importin $\alpha$ and importin $\beta$ again showed no phenotype (Figure 18 A, B).

A

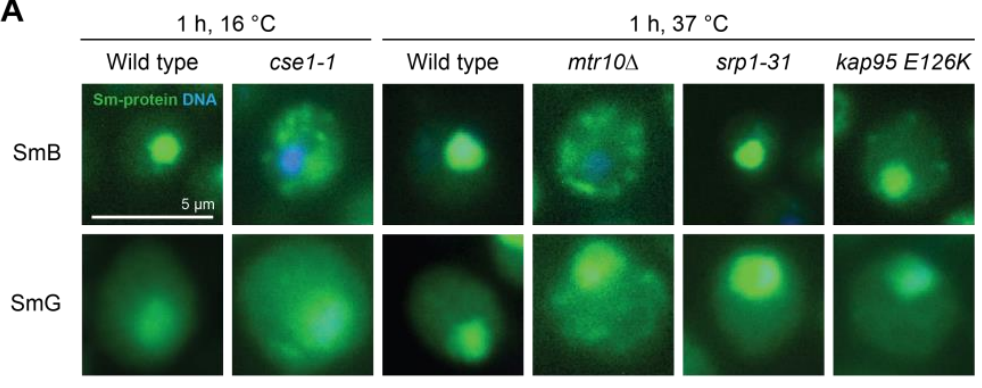

B

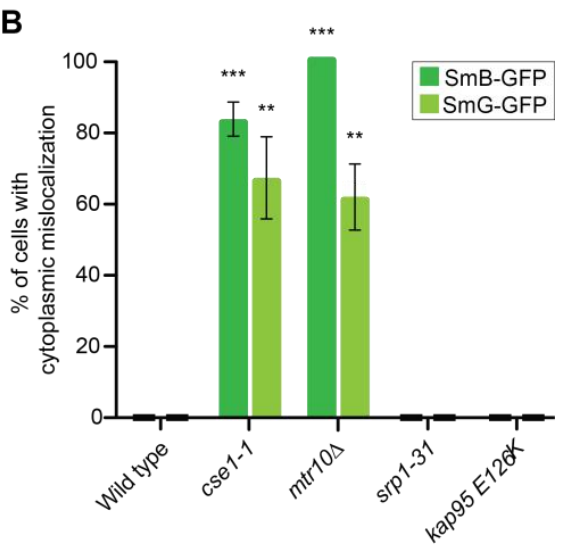

Figure 18: Cse1 and Mtr10 are essential for the correct Sm-ring localization. (A) GFP-microscopy experiments of $c s e 1-1$ and $m t r 10 \Delta$ cells after a $1 \mathrm{~h}$ shift to the indicated temperatures are shown. Cells were transformed with a plasmid containing the indicated GFP-tagged Sm-ring component under its own promotor. (B) Quantification of cells with a cytoplasmic signal shown in (A). The error bars indicate the standard deviation and $p$-values were calculated by an unpaired, one sided student's $t$ test $\left({ }^{*}=p<0.05,{ }^{* *}=p<0.01,{ }^{* * *}=p<0.001\right)$. 
To analyze if the Sm-proteins are only imported into the nucleus when loaded on the snRNAs in the cytoplasm, we investigated the localization of SmB-GFP and SmG-GFP in the snRNA export mutants mex67-5 and xpo1-1. As one can see in Figure 19, there was no change in the localization of $\mathrm{SmB}$ and $\mathrm{SmG}$ in mex67-5, which supports the suggestion, that the Sm-ring components are imported via Cse1 and Mtr10 independent of the snRNAs.

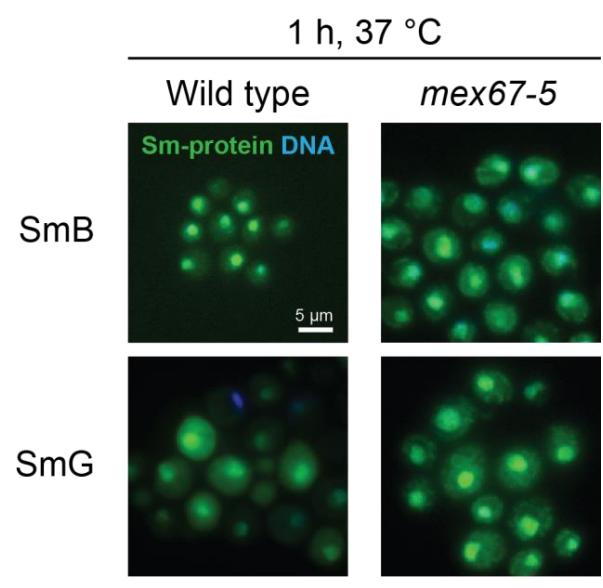

Figure 19: The re-import of the Sm-ring is independent of the snRNAs. GFP-microscopy experiments of mex67-5 cells after $1 \mathrm{~h}$ shift to $37^{\circ} \mathrm{C}$ are shown. Cells were transformed with a plasmid containing the indicated GFP-tagged Sm-ring component under its own promotor.

To analyze the linkage between Sm-ring assembly and shuttling in a different way, we used a strain, which mislocalizes the Sm-ring in the cytoplasm by depletion of SmB together with a partial deletion of SmD1 (Bordonné, 2000).

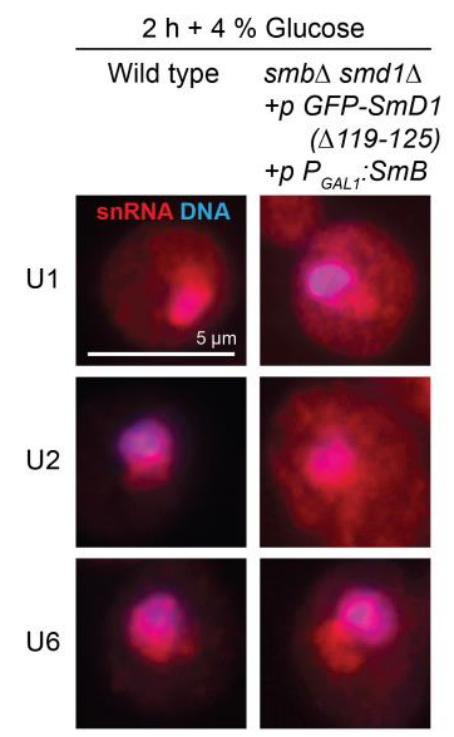

Figure 20: The Sm-ring is essential for snRNA import into the nucleus. Shown are fluorescent in situ hybridization experiments of U1, U2 and U6 in an Sm-ring double mutant strain. Cells were grown in galactose containing medium to the logarithmic phase. Depletion of SmB was performed by adding $4 \%$ glucose to the medium for $2 \mathrm{~h}$ at $25^{\circ} \mathrm{C}$. Cells were fixed with $3.7 \%$ formaldehyde and snRNAs were hybridized with 50 nucleotide long, Cy3-labelled DNA probes (red). The DNA was stained with Hoechst (blue). 
In this double mutant (smb smd1 $+p$ GFP-SmD1(4119-125) $\left.+p P_{G A L 1}: S m B\right)$ we analyzed the snRNA localization. Indeed, a strong cytoplasmic mislocalization of U1 and U2 was observed (Figure 20). U6 showed no cytoplasmic signal, which seems obvious, as U6 contains an Lsm-ring instead of the Sm-ring (Wilusz and Wilusz, 2013).

In summary, we showed that Cse1 and Mtr10 are essential for the Sm-ring import to the nucleus. Our results suggest a co-import of the Sm-ring and the snRNAs and a dependency for proper Sm-ring loading.

\subsection{Cse1 does not bind to snRNAs in vitro but contacts the Sm-ring via SmB}

We have shown that the Sm-ring and the snRNAs are imported to the nucleus together as an snRNP (Figure 20). To investigate how Cse1 contacts the snRNP, we tested if Cse1 is capable of binding the snRNA directly. Although it is rather unlikely that Cse1 binds RNA directly, because of its structure (Cook et al., 2005), we performed in vitro binding studies. We purified recombinant GST-Cse1 from E. coli BL21* cells carrying a $p G E X-6 P-1$ AmpR Ptac:GST-Cse1 plasmid. GST-Cse1 was purified via affinity purification with two $1 \mathrm{ml}$ GSTrap columns using the Äktaprime plus. RNA was eluted from GST-Cse1 with a $1 \mathrm{M} \mathrm{LiCl}$ solution. Western blot analysis shows that most of the protein was not bound to the column (Figure 21A). After the addition of $\mathrm{LiCl}$, there was no elution of proteins, suggesting that no RNA associated complexes were released or present on the column, respectively. In the eluate fractions there was still a decent amount of purified protein left (Figure 21A). Finally, the eluate fractions were pooled and dialyzed and the purity of the protein was controlled via SDS-page with subsequent silver staining (Figure $21 \mathrm{~B}$ ).

A

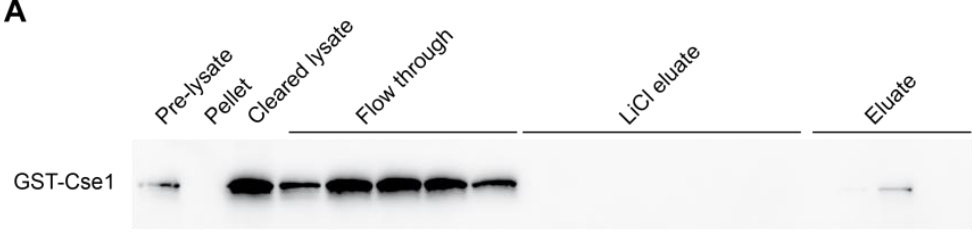

B

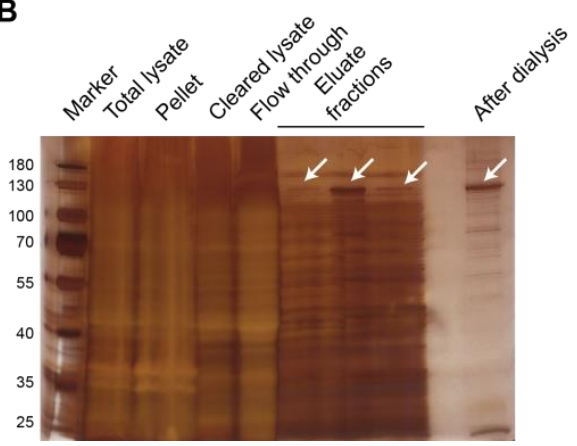

Figure 21: GST-Cse1 purification (A) Western blot analysis of GST-Cse1 from the indicated fractions. GST-Cse1 was detected with an a-GST-antibody. (B) Silver staining of an SDS-gel of selected fractions from GST-Cse1 purification. The Marker indicates the molecular weight (kDa). The white arrowhead marks GST-Cse1 bands.

Previously purified GST by Dr. Claudia Baierlein was used as a negative control. We incubated the recombinant proteins with total RNA purified from wild type cells. Afterwards 
we co-immunoprecipitated the potentially bound RNA and analyzed it via qRT-PCR experiments (Figure 22A). No binding of GST-Cse1 to the snRNAs was detected. Efficient pull down was analyzed by western blot analyses (Figure 22B).

A

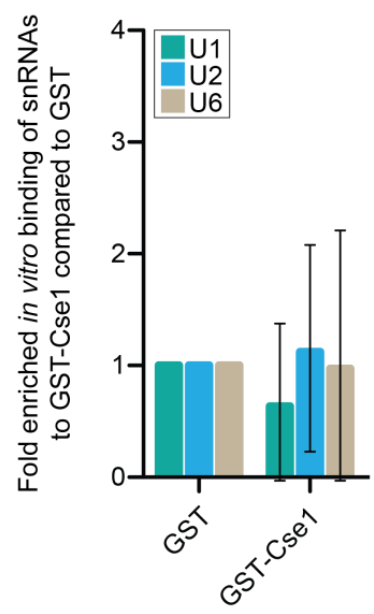

B

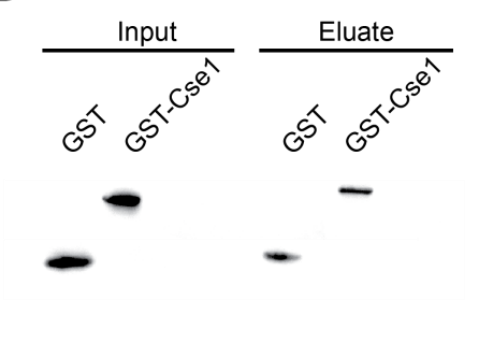

Figure 22: Cse1 does not bind to snRNAs in vitro (A) Recombinant GST-Cse1 was incubated with total RNA and afterwards immunoprecipitated. Bound RNA was isolated and reverse transcribed followed by qRT-PCR. As a negative control recombinant was used. The results clearly show no interaction between Cse1 and U1, U2 and U6. The error bars indicate the standard deviation and $p$ values were calculated by an unpaired, one sided student's t-test $\left({ }^{*}=p<0.05,{ }^{* *}=p<0.01\right.$, $\left.{ }^{* * *}=p<0.001\right)$. (B) Western Blot analysis of the in vitro binding study shown in (A). The successful pulldown of GST and GST-Cse1 is shown.

Because Cse1 did not interact directly with the snRNAs, we analyzed a potential interaction between Cse1 and the Sm-ring component SmB in vivo. We precipitated Cse1-GFP and detected a co-precipitation of $\mathrm{SmB}$ (Figure 23A). These findings suggest that Cse1 might contact the snRNAs via SmB for nuclear import.

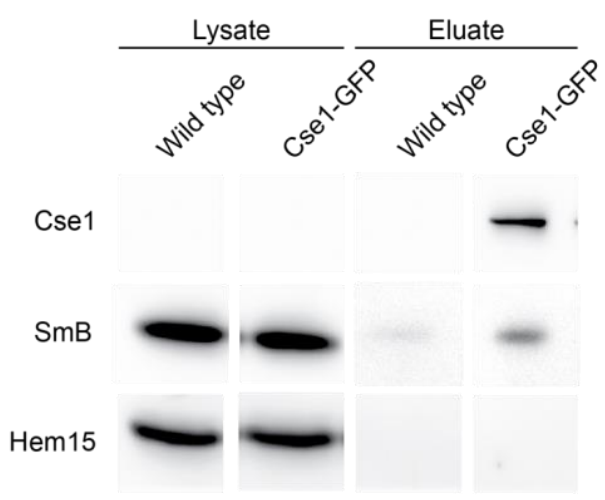

Figure 23: Cse1 interacts with the Sm-ring via SmB. (A) Co-immunoprecipitations reveal a physical interaction between Cse1-GFP and SmB-myc. Cse1-GFP and SmB-myc were detected with antibodies specific for GFP and myc respectively. Hem15 served as a control for unspecific binding to the GFP-Trap beads. 


\subsection{The Sm-ring assembles on the snRNAs in the cytoplasm}

In yeast, it was suggested that the Sm-ring assembly takes place in the cytoplasm and is imported as an RNA-free protein complex (Bordonné, 2000; Matera and Wang, 2014). Our results now indicate a different mode of action: As we have shown that the assembly of the Sm-ring with the snRNAs is essential for the re-import via Cse1 and Mtr10, we propose that the Sm-ring binds the snRNAs in the cytoplasm similar to higher eukaryotes. Nevertheless, a binding of the Sm-ring to the snRNAs prior to their nuclear export is still conceivable. Therefore, we trapped the newly synthesized snRNAs in the nucleus and performed RIP experiments with GFP-tagged SmB in the snRNA export mutants xpo1-1, mex67-5 and the double mutant xpo1-1 mex67-5. The interaction of U1 and U2 with SmB was significantly decreased in all three strains. Only 6-10\% of the interaction compared to wild type remained (Figure 24A). This shows that the snRNAs cannot interact with the Sm-ring before nuclear export, even though SmB and SmG are located to the nucleus in xpo1-1, mex67-5 and the double mutant (see also Figure 19).

A

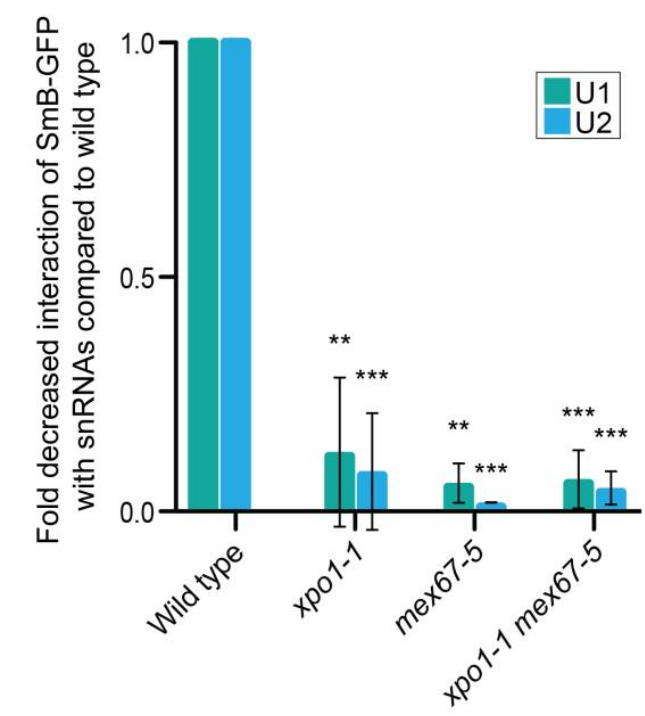

B

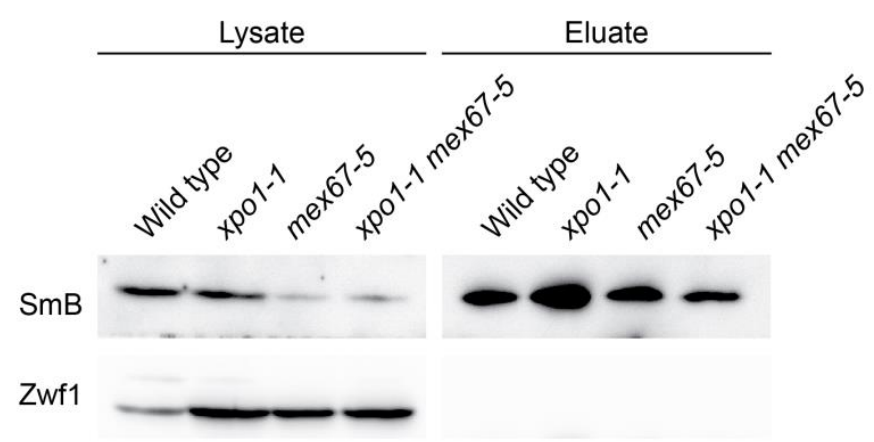

Figure 24: The Sm-ring assembles on the snRNAs after nuclear export in the cytoplasm.

(A) RIP-experiments show a decreased interaction between SmB-GFP and U1 and U2 in the snRNA export mutants xpo1-1, mex67-5 and the double mutant xpo1-1 mex67-5. Indicated strains were shifted to $37^{\circ} \mathrm{C}$ for $1 \mathrm{~h}$ prior to cell lysis and immunoprecipitation. Co-immunoprecipitated RNA was used for qRT-PCR analyses. The error bars indicate the standard deviation and $p$-values were calculated by an unpaired, one sided student's t-test $\left({ }^{*}=p<0.05,{ }^{* *}=p<0.01,{ }^{* * *}=p<0.001\right)$. (B) Western blot analysis of immunoprecipitated proteins from RIP experiments shown in (A). Zwf1 served as a control for unspecific protein binding to the GFP-trap beads. Indicated GFP-tagged proteins were detected with a GFP-specific antibody.

Together, these data indicate that the Sm-ring assembles on the snRNAs in the cytoplasm. Afterwards, Cse1 contacts the Sm-ring via SmB to import the snRNP to the nucleus. 


\subsection{Nucleo-cytoplasmic shuttling is essential for $\mathrm{U} 1$ processing}

After transcription, the U1 transcript is processed by the endoribonuclease Rnt1 and the nuclear exoribonuclease Rrp6 at its 3'-end. For this processing step the binding of the Sm-ring is an essential prerequisite (Coy et al., 2013; Seipelt et al., 1999; Shukla and Parker, 2014). In S. cerevisiae, Rnt1 is located to the nucleus (Catala et al., 2004). Therefore, U1 processing occurs in the nucleus. We addressed the question whether $\mathrm{U} 1$ is processed before or after the nucleo-cytoplasmic shuttling. Thus, we measured the ratio of unprocessed U1 to total U1 in the two nuclear import mutants cse 1-1, mtr104 and the snRNA export mutant xpo1-1 mex675. Clearly, the depletion of the snRNAs from the nucleus in cse1-1 and mtr104 resulted in a strong accumulation of unprocessed U1 (Figure 25), which indicated that Rnt1 and Rrp6 did not process the cytoplasmic U1 snRNA prior to export. In csel-1 we detected nearly a fivefold enrichment of unprocessed U1 compared to wild type. For mtr10 the change was less strong (twofold change), but still significant. Surprisingly, the double export mutant xpo1-1 mex67-5 did also show a severe processing defect. This indicates that the retention of $\mathrm{U} 1$ in the processing compartment alone is not sufficient for processing and rather requires the association of the Sm-ring in the cytoplasm. Therefore, shuttling through the cytoplasm seems to be needed for correct U1 3'-end processing.

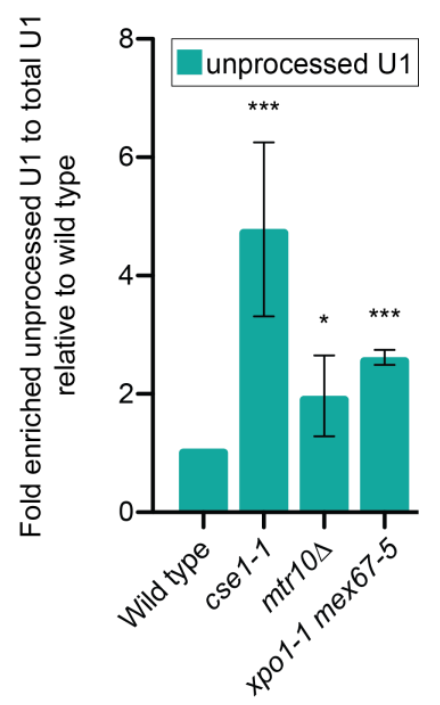

Figure 25: Unprocessed U1 accumulates in snRNA transport mutants. Interruption of the nucleocytoplasmic shuttling of snRNAs results in processing defects of U1. Total RNA from the indicated strains was isolated after the cells were shifted for $1 \mathrm{~h}$ to the restrictive temperature $\left(16^{\circ} \mathrm{C}\right.$ for $\operatorname{cse} 1-1$ and $37^{\circ} \mathrm{C}$ for mtr10 $\Delta$ and xpo1-1 mex67-5). The fold enriched unprocessed U1 compared to total U1 in the indicated strains relative to wild type is shown. The error bars indicate the standard deviation and $p$-values were calculated by an unpaired, one sided student's t-test $\left({ }^{*}=p<0.05,{ }^{* *}=p<0.01\right.$, $\left.{ }^{* \star *}=p<0.001\right)$. 


\subsection{TMG-capping terminates snRNA shuttling}

In human cells, the addition of the TMG cap by TGS1 is the last cytoplasmic snRNA processing step before the Ran-dependent nuclear import via SPN and importin $\beta$ occurs (Matera and Wang, 2014). In S. cerevisiae, the homologues protein Tgs1 is located to the nucleolus and is essential for the cap-hypermethylation of snRNAs and snoRNAs (Mouaikel et al., 2002). This raised the question, if the snRNAs are trimethylated before or after the nucleo-cytoplasmic shuttling. We performed RIP experiments with a TMG-cap specific antibody. We isolated total RNA from the two import mutants cse $1-1$ and mtr104. As a positive control we used a strain lacking TGS1. Interestingly, for both import deficient strains the values were comparable to $\operatorname{tgs} 1 \Delta$. The remaining pull down of snRNAs in $\operatorname{tgs} 1 \Delta$ and the tested import mutants was due to unspecific monomethylcap binding of the antibody because no TMG-capped snRNAs are present in $\operatorname{tg} 1 \Delta$ (Mouaikel et al., 2002). Thus, tgs1 1 reflected the amount of the unspecific binding of the antibody. Since in both cases we could only detect around 30 to $50 \%$ of trimethylated snRNAs (Figure 26), it is most likely that the trimethylation of U1 and U2 occurs after the nucleo-cytoplasmic shuttling.

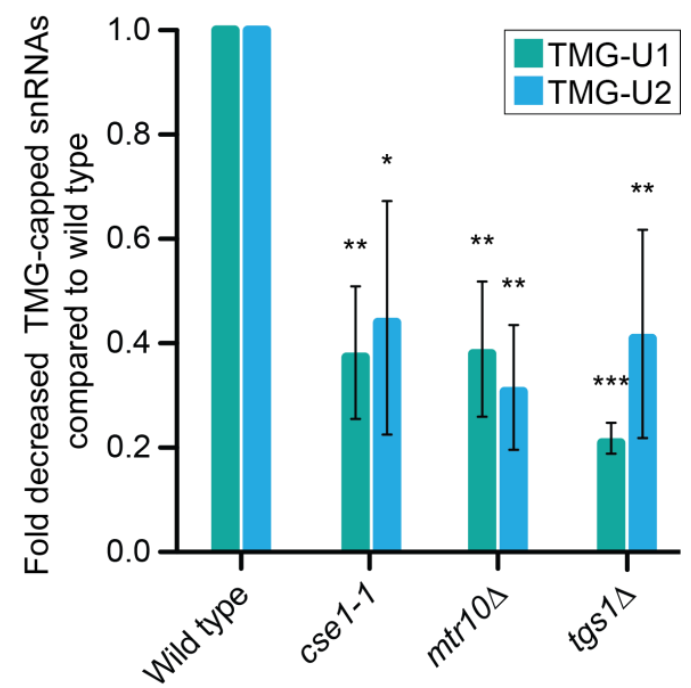

Figure 26: TMG-capping of snRNAs is reduced in import mutants. Interruption of the import of snRNAs results in decreased TMG-capping of U1 and U2. Total RNA from the indicated strains was isolated after a $1 \mathrm{~h}$ shift to restrictive temperature $\left(16{ }^{\circ} \mathrm{C}\right.$ for $\operatorname{cse} 1-1$ and $37^{\circ} \mathrm{C}$ for $m \operatorname{tr} 10 \Delta$ and $\operatorname{tgs} 1 \Delta$ ). Total RNA was incubated with a TMG-specific antibody and the immunoprecipitated RNA was analysed by qRT-PCR. The fold decreased TMG-capped snRNA in the indicated strains relative to wild type is shown. The error bars indicate the standard deviation and $p$-values were calculated by an unpaired, one sided student's t-test $\left({ }^{*}=p<0.05,{ }^{* *}=p<0.01,{ }^{* * *}=p<0.001\right)$.

\section{9 $\mathrm{SmB}$ is involved in the trimethylation of snRNAs}

Our previous results indicated that trimethylation of the snRNA-cap occurs after nucleocytoplasmic shuttling. In addition, we showed that the snRNAs are already bound to the Smring when they enter the nucleus. Previous studies detected an in vitro binding of $\mathrm{SmB}$ to 
Tgs1 (Mouaikel et al., 2002). Therefore, we investigated the in vivo interaction between SmB and Tgs1 and the localization of the snRNAs in smbL. These experiments address the question whether the assembly of the snRNP is required for trimethylation to occur.

To investigate the snRNAs localization in cells depleted for $S M B$, we used a strain lacking $S M B$ that is covered by a plasmid encoding $P_{G A L 1}: S m B$ (Bordonné, 2000). This allowed the depletion of SmB through addition of $4 \%$ glucose to the growth medium for $2 \mathrm{~h}$. To be able to detect a nucleolar co-localization we used an Atto488-labeled probe against the internal transcribed spacer 2 (ITS2) of the pre-rRNA, which is localized to the nucleolus.

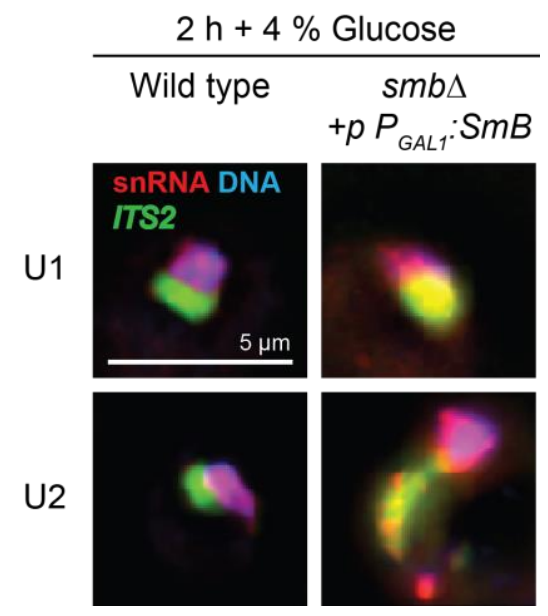

Figure 27: Depletion of SmB results in a nucleolar mislocalization of U1 and U2. Shown are fluorescent in situ hybridization experiments of $\mathrm{U} 1$ and $\mathrm{U} 2$ in $s m b \Delta+p P_{G A L 1}: S m B$. Cells were grown in galactose containing medium to the logarithmic phase. Depletion of $\mathrm{SmB}$ was initiated by addition of $4 \%$ glucose to the medium for $2 \mathrm{~h}$ at $25^{\circ} \mathrm{C}$. Cells were fixed with $3.7 \%$ formaldehyde and snRNAs were localized through FISH with 50 nucleotide long, Cy3-labelled DNA probe (red). ITS2 as nucleolar marker was stained with a 50 nucleotide long Atto488-labelled DNA-probe (green). The DNA was stained with Hoechst (blue).

In the wild type there was no overlay (yellow) of the snRNA signal (red) with the ITS2 signal (green) detectable. In contrast, we could see a very strong nucleolar accumulation of U1 and $\mathrm{U} 2$ in $s m b \triangle+\mathrm{p} P_{G A L 1}: S M B$ after $2 \mathrm{~h}$ depletion of $\mathrm{SmB}$ (Figure 27). To further confirm the role of $\mathrm{SmB}$ in snRNA trimethylation, we invetigated the interaction between $\mathrm{SmB}$ and Tgs1 in vivo. In contrast to prior findings, where no in vivo interaction between $\mathrm{SmB}$ and Tgs1 was detected (Mouaikel et al., 2002), our experiments demonstrate that Tgs1 and SmB physically interact in vivo (Figure 28). The results reveal the importance of $\mathrm{SmB}$ in the trimethylation process, as the depletion of $\mathrm{SmB}$ results in the loss of correct snRNP release from the nucleolus (Figure 27). 


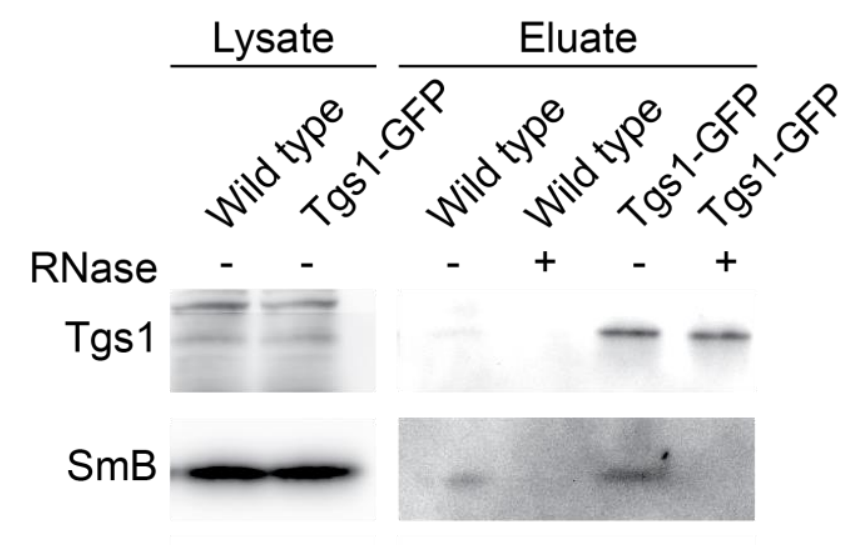

Hem15

Figure 28: Tgs1 and SmB physically interact in vivo. Co-immunoprecipitation experiments reveal a physical interaction between Tgs1-GFP and SmB-myc. Tgs1-GFP and SmB-myc were detected with antibodies against GFP or myc. Hem15 served as a control for unspecific binding to the GFP-Trap beads.

\subsection{TMG-capped snRNAs preferentially interact with spliceosomal components and not with export factors}

Interestingly, it has been demonstrated that the deletion of TGS1 results in an increased association of the CBC to the U1 snRNP (Schwer et al., 2011). In addition, it has been shown that the cold sensitive growth defect of $\operatorname{tgs} 1 \Delta$ can be rescued by mutations in the RNAbinding domain of Cbp20 (Schwer et al., 2011). Thus, it is conceivable that the trimethylation of $\mathrm{U} 1$ and $\mathrm{U} 2$ finalizes snRNA shuttling. If TGS1 is deleted, the $\mathrm{m}^{7} \mathrm{G}$-capped RNA and its bound CBC will repeatedly be recognized as export cargo. Due to the fact that Xpo1 contacts the snRNP via an unknown protein, it is possible that it contacts the CBC directly, which we investigated by co-immunoprecitation experiments.

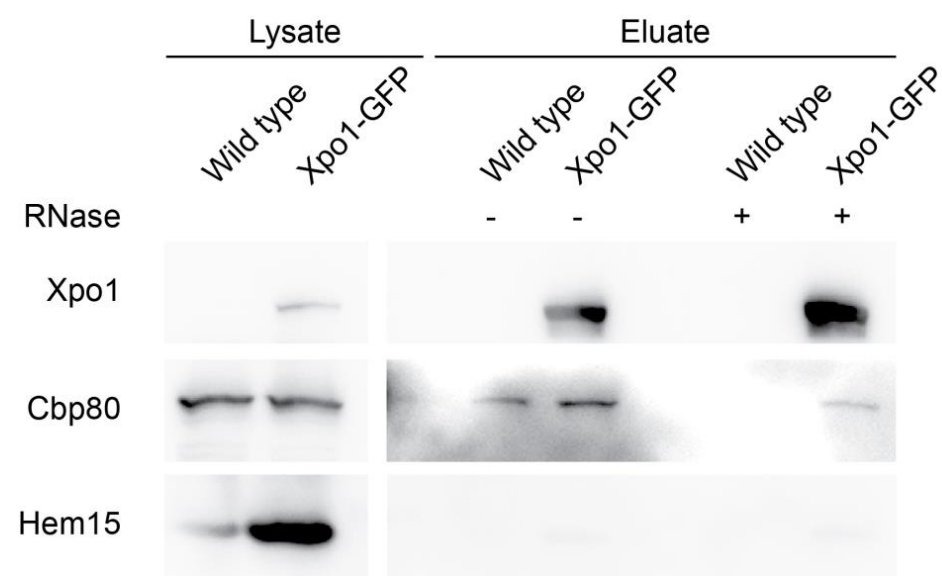

Figure 29: Xpo1 interacts with the cap binding complex in vivo. Co-immunoprecipitation experiments reveal a physical interaction between Xpo1-GFP and Cbp80-myc. Xpo1-GFP and Cbp80myc were detected with antibodies specific for GFP and myc, respectively. Hem15 served as a control for unspecific binding to the GFP-Trap beads. 
Indeed, as shown in Figure 29 a faint but clear interaction between Xpo1 and Cbp80 was detectable, suggesting that the export factor contacts the CBC-bound cap of snRNAs.If the theory is correct and TMG-capped snRNAs do not shuttle anymore, Mex67 and Xpo1 should not contact the TMG-capped snRNA. Thus, we analyzed the physical interaction of the export receptors Mex67 and Xpo1 to TMG-capped snRNAs in relation to the splicing factors Prp40 and Prp17. Prp40 binds to all snRNAs, while Prp17, as it is a late splicing factor, solely binds to U2, U5 and U6 (Sapra et al., 2008). Hence, as the binding of U1 to Prp17 served as a negative control, we should not be able to detect any interaction between them. We therefore performed a two-step RIP experiment, in which we first precipitated Prp40-GFP, Prp17-GFP, Mex67-GFP and Xpo1-GFP and isolated the co-immunoprecipitated RNA. Secondly, we incubated this RNA with an $\alpha$-TMG-antibody and again isolated the immunoprecipitated RNA for reverse transcription and qRT-PCR analysis. The pulldown of the GFP tagged proteins was analyzed in western blot analyses (Figure 30B).

A

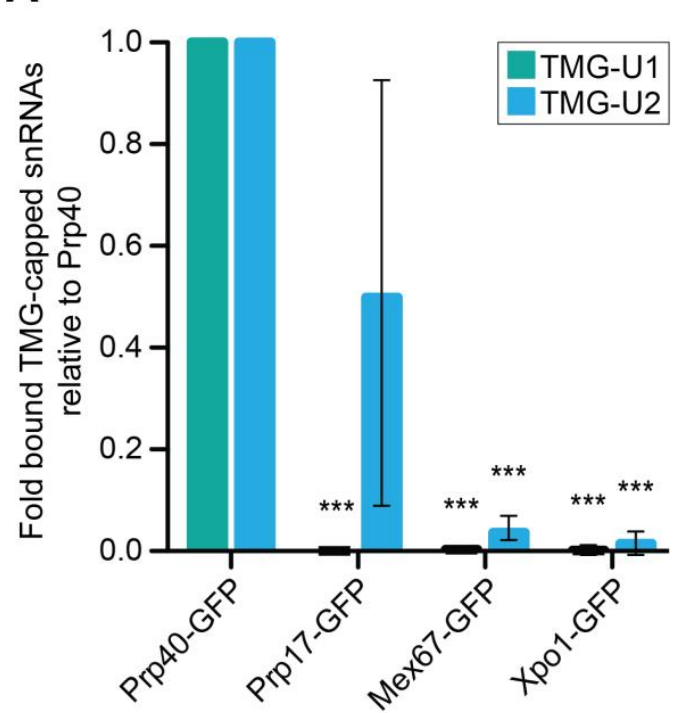

B

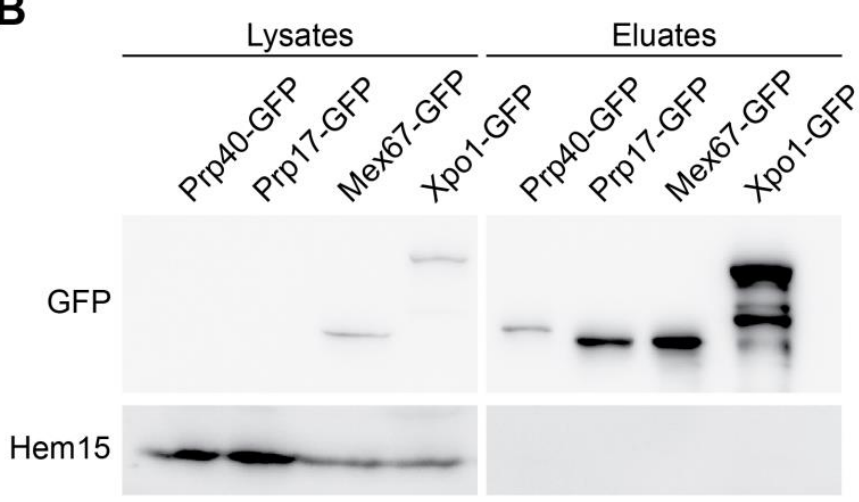

Figure 30: TMG-capped snRNAs preferentially bind to splicing factors. (A) Two step RIPexperiments with GFP tagged splicing factors Prp17 and Prp40 and the export receptors Mex67 and Xpo1 are shown. First, the immunoprecipitated RNA from the RIP experiments with the indicated proteins was isolated. Secondly, this RNA was incubated with an TMG-specific antibody and used for immunoprecipitations of TMG-capped RNA. Afterwards, qRT-PCR analyses followed. The error bars indicate the standard deviation and $p$-values were calculated by an unpaired, one sided student's $t$ test $\left.{ }^{*}=p<0.05,{ }^{* *}=p<0.01,{ }^{* * *}=p<0.001\right)$. (B) Western blot analysis of immunoprecipitated proteins from RIP experiments shown in (A). Hem15 served as a control for unspecific protein binding to the GFP-trap beads. Indicated GFP-tagged proteins were detected with a GFP-specific antibody.

We also performed this experiment with a no tag control in which we could not detect any RNA. Thus, we did not get any $\mathrm{C}_{\mathrm{q}}$-values for the no tag control and did not include it in our figure, but rather display our results relative to Prp40-GFP (Figure 30A). The interaction between TMG-U2 and Prp17 is $50 \%$ lower than the interaction of TMG-U2 and Prp40. In 
contrast, the interaction between TMG-U2 and Mex67 ( 4\%) and Xpo1 ( 1\%) is nearly absent. As U1 does not interact with Prp17, we could not detect any interaction between TMG-U1 and Prp17. This shows that the snRNA-pulldown was specific. Additionally, we measured only $\sim 1 \%$ relative binding of TMG-U1 to Mex67 and Xpo1. Taken together, these results indicate that the TMG-capped snRNAs U1 and U2 preferentially bind to splicing factors and not to Mex67 and Xpo1. Therefore, we conclude that trimethylation of snRNAs finalizes nucleo-cytoplasmic shuttling.

\subsection{Depletion of snRNAs from the nucleus results in splicing defects}

We could show that the nucleo-cytoplasmic shuttling is essential for the correct snRNA processing and the snRNP maturation. Any interruption of this cycle leads to incorrect snRNAs and/or snRNPs. Therefore, we wanted to investigate why nucleo-cytoplasmic shuttling of snRNA in general evolved in eukaryotes.

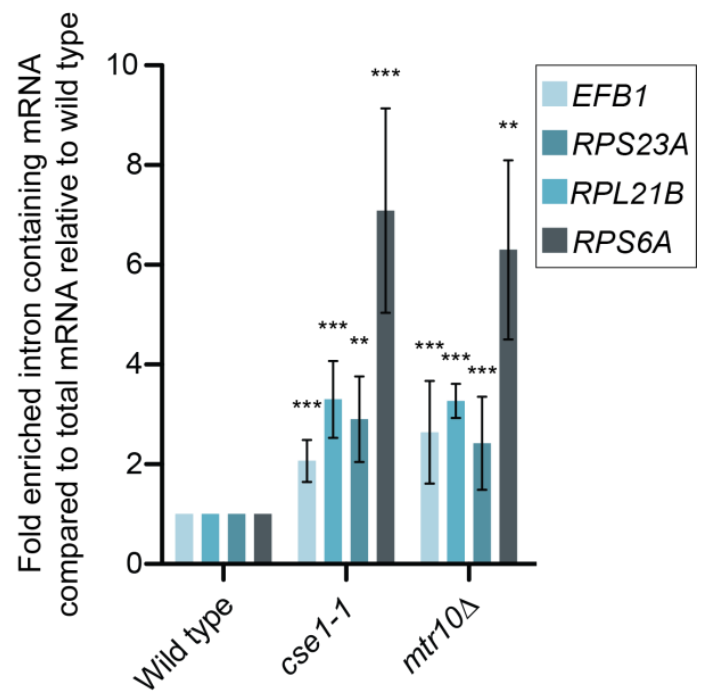

Figure 31: Depletion of snRNAs from the nucleus results in splicing defects. Interruption of the import of snRNAs results in splicing defects of intron-containing transcripts. Total RNA from the indicated strains was isolated after a $1 \mathrm{~h}$ shift to restrictive temperatures $\left(16^{\circ} \mathrm{C}\right.$ for cse $1-1$ and $37^{\circ} \mathrm{C}$ for mtr104). Total RNA was analysed by qRT-PCR. The fold increased intron containing mRNA compared to total mRNA in the indicated strains relative to wild type is shown. The error bars indicate the standard deviation and $p$-values were calculated by an unpaired, one sided student's t-test $\left({ }^{*}=\right.$ $\left.p<0.05,{ }^{* *}=p<0.01,{ }^{* * *}=p<0.001\right)$.

We depleted the snRNAs from the nucleus by trapping them in the cytoplasm in the nuclear import mutants. After shifting cse1-1 and mtr104 to their non-permissive temperatures, we isolated the total RNA. This allowed us to measure the relative splicing rates of several selected intron containing transcripts (EFB1, RPS23A, RPL21B and RPS6A).We compared the intron containing mRNA to total mRNA to exclude potential errors that might occur due to possible different mRNA stabilities in the different strains. Indeed, we observed a strong accumulation of intron containing mRNAs in the cells (Figure 31). In cse1-1 we detected 
seven-fold enrichment of the intron-containing RPS6A mRNA. The three other selected transcripts $E F B 1, R P S 23 A$ and $R P L 21 B$ were enriched between two- and four-fold. A similar result was obtained for mtr104, clearly showing that the depletion of the snRNAs from the nucleus and the spliceosome results in splicing defects.

\subsection{Immature $U 1$ is incorporated into the spliceosome and induces splicing defects}

The export block of the snRNAs in mex67-5 results in processing defects of U1 (Figure 25), because U1 processing takes place after the nucleo-cytoplasmic shuttling, as we could show before (see 4.7). Therefore, we investigated the physical interaction of Prp40-GFP with U1 and unprocessed U1 in wild type and in mex67-5. Strikingly, we found that Prp40 shows a 40 fold enriched binding to unprocessed U1 in mex67-5 compared to wild type (Figure 32A), showing that immature U1 is integrated into spliceosomes. Western blot analysis of the immunoprecipitated proteins is shown in Figure 32B.

A

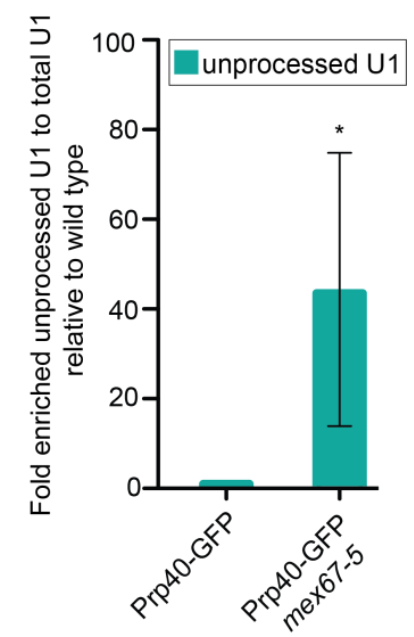

B

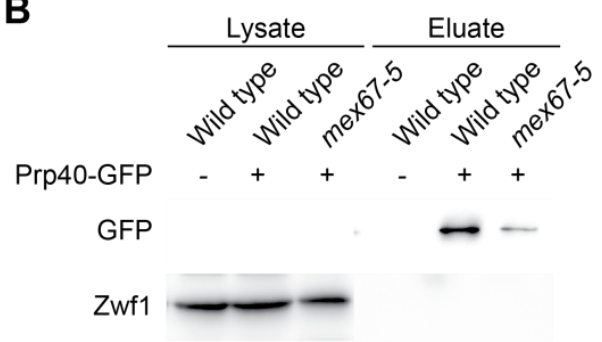

Figure 32: An export block of snRNAs results in the incorporation of faulty processed U1 into the spliceosome. (A) RIP-experiments show an increased interaction between Prp40-GFP and unprocessed $\mathrm{U} 1$ compared to total $\mathrm{U} 1$ in the snRNA export mutant mex67-5 relative to wild type. The indicated strains were shifted to $37^{\circ} \mathrm{C}$ for $1 \mathrm{~h}$ prior to cell lysis and immunoprecipitation. Coimmunoprecipitated RNA was used in qRT-PCR analyses. The error bars indicate the standard deviation and $p$-values were calculated by an unpaired, one sided student's t-test $\left({ }^{*}=p<0.05\right.$, $\left.{ }^{* *}=p<0.01,{ }^{* * *}=p<0.001\right)$. (B) Western blot analysis of immunoprecipitated proteins from RIP experiments shown in (A). Zwf1 served as a control for unspecific protein binding to the GFP-trap beads. Indicated GFP-tagged proteins were detected with a GFP-specific antibody.

To determine the percentage of affected spliceosomes, we calculated the ratio of unprocessed U1 to total U1 bound to Prp40 in wild type and mex67-5 (Figure 33). While in wild type only $\sim 0.5 \%$ of the measured Prp40 bound U1 was unprocessed, we detected $\sim 29 \%$ of the U1 RNA to be immature in mex67-5. 


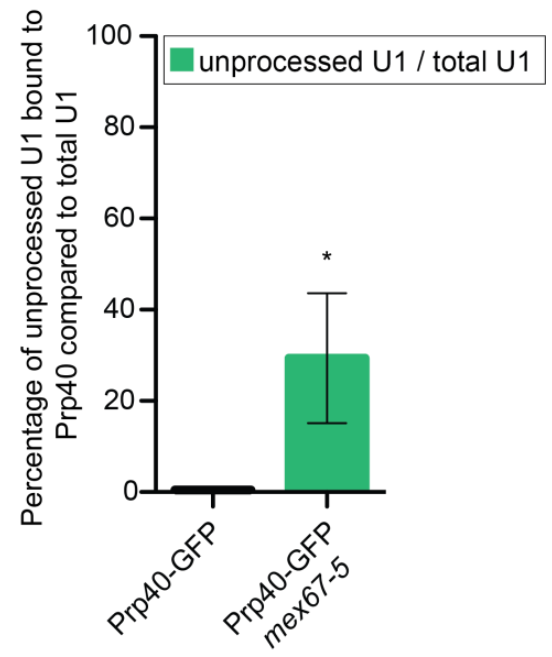

Figure 33: Nearly one third of spliceosomes contain immature U1 snRNAs in mex67-5. RIPexperiments shown in Figure 32 were used to calculate the percentage of faulty processed U1 containing spliceosomes. The error bars indicate the standard deviation and $p$-values were calculated by an unpaired, one sided student's t-test $\left({ }^{*}=p<0.05,{ }^{* *}=p<0.01,{ }^{* * *}=p<0.001\right)$.

These results show that immature snRNAs can be integrated into spliceosomes. Therefore, we investigated the physiological consequences and the interaction of intron containing transcripts with Prp40 in mex67-5. Interestingly, we detected a significant enrichment for three of the four transcripts (Figure 34), indicating that the presence of immature U1 in the spliceosomes leads to the presence of more intron containing transcripts in Prp40-containing spliceosomes, which suggests that immature spliceosomes do not function correctly.

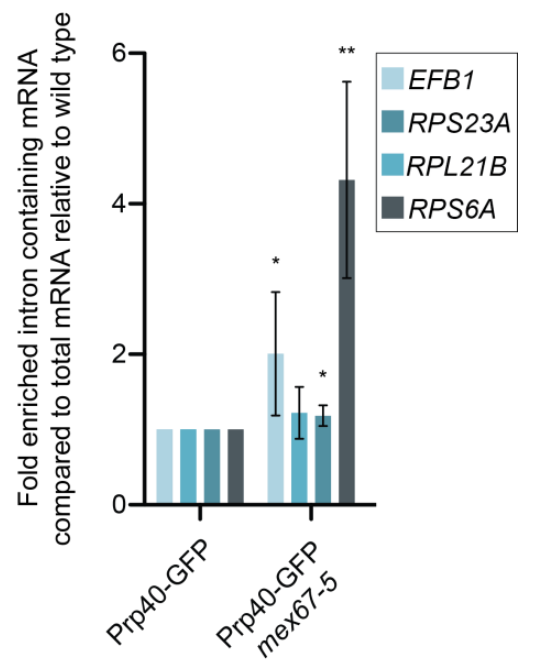

Figure 34: Intron containing transcripts are enriched in spliceosomes that contain immature snRNAs. Co-immunoprecipitated and reverse transcribed RNA from the previous experiment (Figure 32) was used for this analysis. Prp40-GFP interaction with intron containing transcripts is significantly enriched in mex67-5 relative to wild type. The error bars indicate the standard deviation and $p$-values were calculated by an unpaired, one sided student's t-test $\left({ }^{*}=p<0.05,{ }^{* *}=p<0.01,{ }^{* * *}=p<0.001\right)$.

Consequently, we should be able to detect general splicing defects in the export mutants. We performed qRT-PCR experiments in xpo1-1, mex67-5 and the double mutant. We compared 
the intron containing mRNA with the total mRNA in relation to wild type, using the same transcripts as shown in Figure 31A and Figure 34. The amount of the intron containing mRNAs compared to the total mRNA was increased for all tested mRNAs (Figure 35). In all strains the enrichment was between 2- and 4.5-fold.

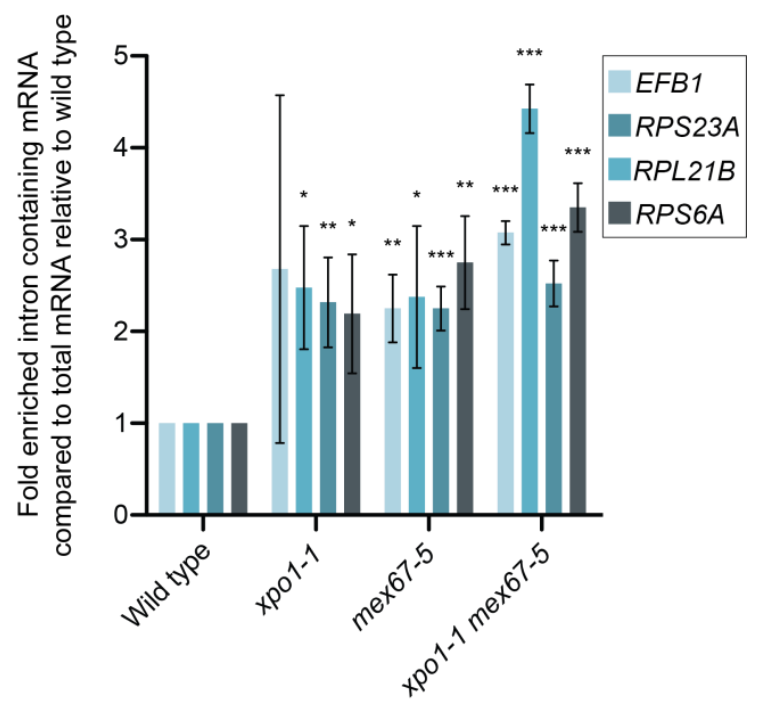

Figure 35: snRNA export mutants show severe splicing defects. Blocking the export of snRNAs results in splicing defects of intron-containing transcripts. Total RNA from the indicated strains was isolated after the cells were shifted to a restrictive temperature $\left(37^{\circ} \mathrm{C}\right)$ for $1 \mathrm{~h}$. Total RNA was analyzed by qRT-PCR. The fold increased intron containing mRNA compared to total mRNA in the indicated strains relative to wild type is shown. The error bars indicate the standard deviation and $p$ values were calculated by an unpaired, one sided student's t-test $\left({ }^{*}=p<0.05,{ }^{* *}=p<0.01\right.$, $* * *=p<0.001)$.

\subsection{3 snRNA export block in mex67-5 xpo1-1 results in severe genome-wide splicing defects}

To verify the previous results and to gain insights into genome-wide effects of snRNA export defects, we performed RNA-Sequencing studies in the snRNA export deficient double mutant mex67-5 xpo1-1. We isolated total RNA from mex67-5 xpo1-1 cells after a $1 \mathrm{~h}$ temperature shift to the non-permissive temperature $\left(37^{\circ} \mathrm{C}\right)$. Afterwards, poly-adenylated RNA was sequenced and raw data analysis was performed by Dr. Thomas Lingner and Dr. Gabriela Salinas in the Microarray and Deep-Sequencing Facility Göttingen. We calculated the ratio of intronic reads compared to exonic reads. The data showed nearly five times more intronic reads in mex67-5 xpo1-1 than in wild type (Figure 36A). In total, 144 introns were detected, of which 115 were significantly enriched. $67 \%$ of all introns were enriched at least two-fold (Figure 36B). 
A

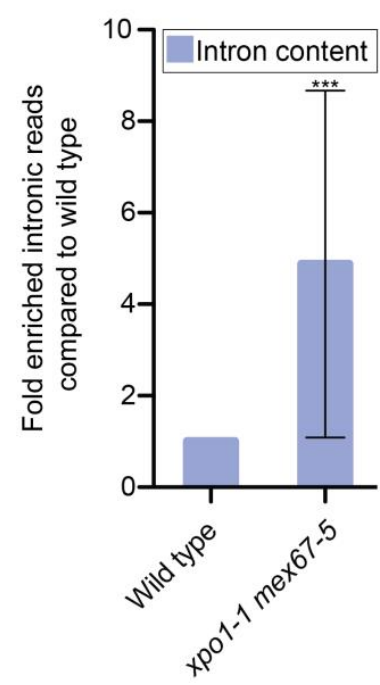

B

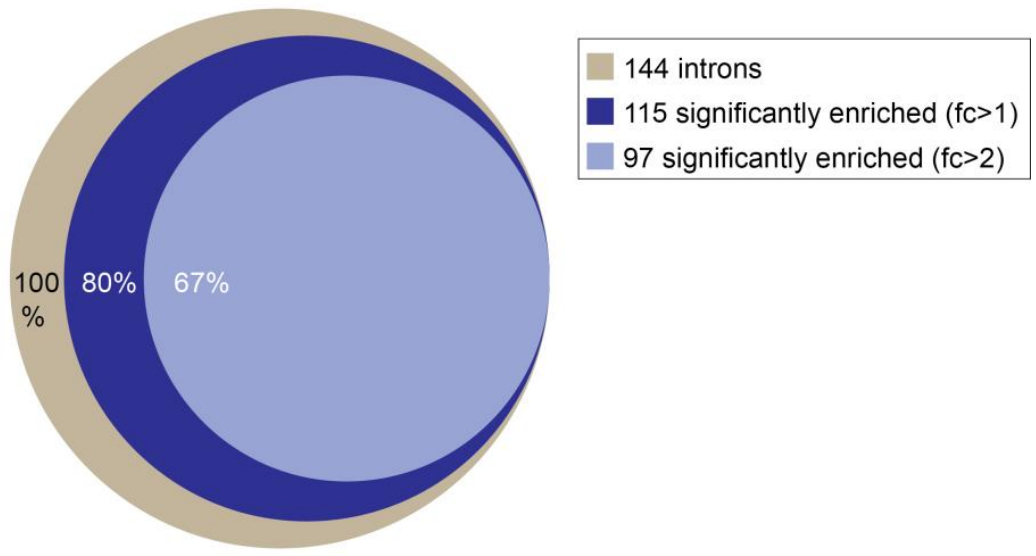

Figure 36: RNA-Sequencing in mex67-5 xpo1-1 reveals genome wide splicing defects. (A) Blocking snRNA export results in genome wide splicing defects of intron-containing transcripts. Total RNA from the indicated strains was isolated after the cells were shifted to a restrictive temperature $\left(37^{\circ} \mathrm{C}\right)$ for $1 \mathrm{~h}$. Total RNA was analysed by RNA-Sequencing by Dr. Thomas Lingner and Dr. Gabriela Salinas in the Microarray and Deep-Sequencing Facility Göttingen. The fold increased intronic reads compared to total wild type is shown. The error bars indicate the standard deviation and $p$-values were calculated by an unpaired, one sided student's t-test $\left({ }^{*}=p<0.05,{ }^{* \star}=p<0.01,{ }^{* * *}=p<0.001\right)$. (B) Statistical analysis of all intronic reads from the RNA-Sequencing experiments.

The significantly changed intronic reads were analyzed in more detail. We sorted the introns from highest fold change to lowest and annotated the corresponding genes and functional groups (Figure 37). Only three of the 115 introns had lesser intronic counts in mex67-5 xpo1-1 compared to wild type. There was no functional group showing visible increased intronic reads compared to the others, indicating that the splicing defect is rather general.

Taken together, the results of this work show that (i) snRNAs shuttle in yeast. We identified Mex67 and Xpo1 as snRNA exportins and Cse1 and Mtr10 as importins. (ii) The Sm-ring assembly takes place in cytoplasm and is a prerequisite for nuclear snRNA processing. (iii) TMG-capping involves SmB and finalizes nucleo-cytoplasmic shuttling. (iv) Immediate nuclear export of immature snRNAs is essential for the correct assembly of the splicing machinery and splicing. 


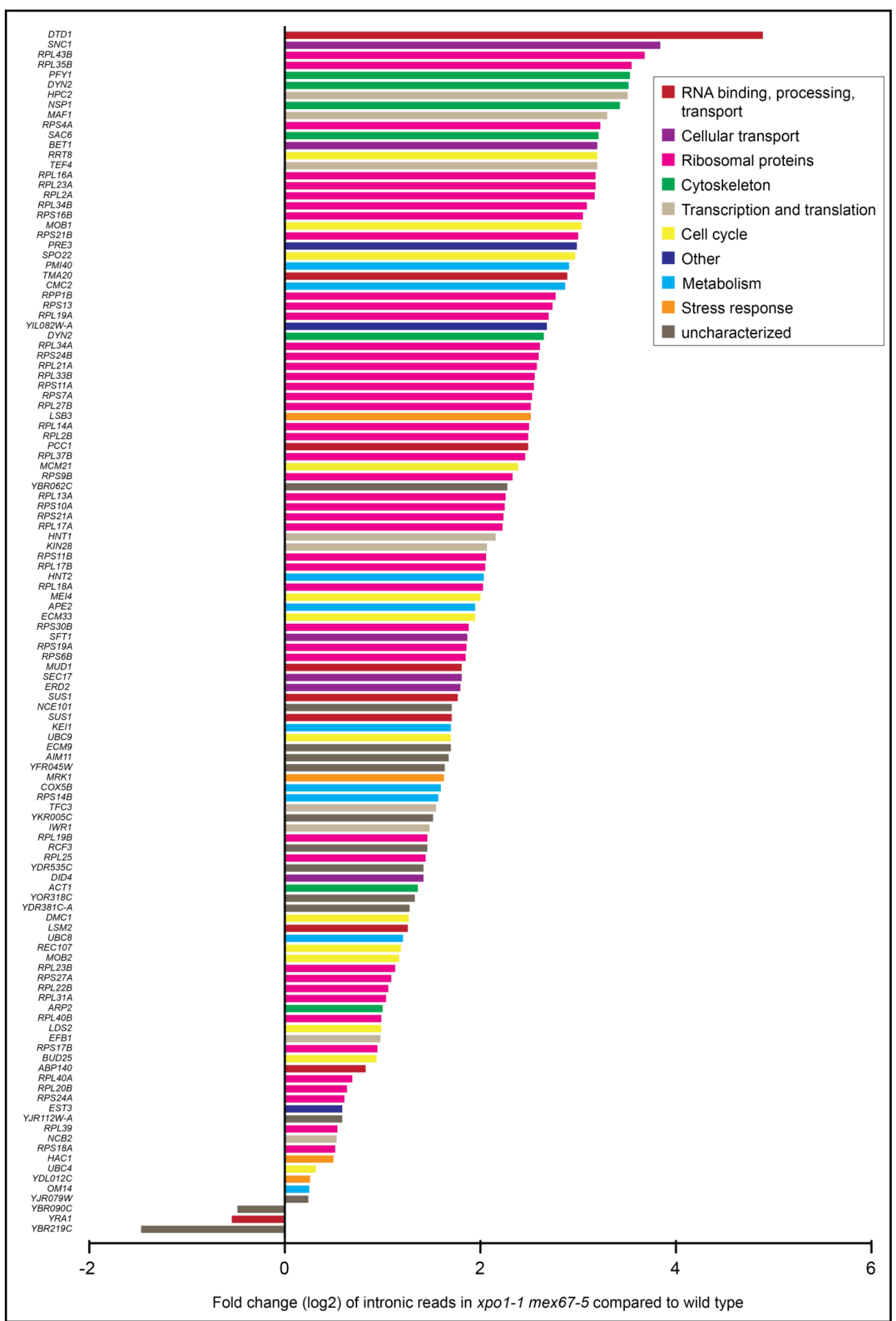

Figure 37: Detailed view of the RNA-Sequencing analysis of intron containing transcripts in mex67-5 xpo1-1. The RNA-Sequencing study reveals an overall enrichment of unspliced mRNAs. All significantly changed introns are listed and the functional groups are indicated. 


\section{Discussion}

Nucleo-cytoplasmic shuttling of snRNAs in higher eukaryotes is a well investigated process (Matera and Wang, 2014; Sloan et al., 2016). For processing and snRNP assembly, snRNAs undergo a cytoplasmic phase before they are imported into the nucleus. However, in $S$. cerevisiae it remained unsolved whether snRNAs shuttle. Earlier work provided the first evidence for shuttling of yeast snRNAs via a heterokaryon assay. However, the authors declared their observation to be an artifact (Olson and Siliciano, 2003). The controversy why functional RNAs, that assemble as RNPs often undergo a nucleo-cytoplasmic shuttling is still ongoing. The favored explanation is the separation of the site of RNP assembly and the site of RNP function to diminish aberently processed RNAs in RNPs, because most non-coding RNAs need to be processed after transcription to be fully functional and shuttling is thus a common theme. However, up to date there is no experimental evidence for such a model. Therefore, in the present study, we set out to analyze whether snRNAs also shuttle in yeast and to investigate the physiological role of such a transport process.

\section{1 snRNAs require general mRNA export factors for nuclear export}

Earlier, it was shown that the non-coding telomerase RNA TLC1 is exported to the cytoplasm by the mRNA export factor Mex67 and Xpo1 (Gallardo et al., 2008; Wu et al., 2014). In addition, Xpo1 has been identified to transport rRNA (Moy and Silver, 2002) and in human cells the Xpo1 homologue CRM1 transports snRNAs into the cytoplasm via interaction with the adaptor protein PHAX (Ohno et al., 2000). Therefore, we first examined the influence of mutants of MEX67 and XPO1 on the localization of snRNAs.

As the steady state localization of snRNAs is nuclear, we used an approach to visualize snRNAs predominantly in the cytoplasm, so that an accumulation in the nucleus is possible to be detected. This was feasible by creating relatively large (100-450 nt) DIG-labeled RNA probes that are less likely to penetrate the nuclear envelope. We observed dot-like accumulations of snRNAs in all export mutant strains (mex67-5, xpo1-1, mex67-5 xpo1-1, rat7-1 and rat8-2) indicating the necessity of these factors for the nuclear export of snRNAs (Figure 11). The export of human snRNAs via the PHAX-CRM1 export complex is dependent on the CBC (Izaurralde et al., 1995). Our results provide first evidence for a CBCmediated export pathway of Sm-class snRNAs in yeast. Interestingly, we could not visualize any effect for U6 in xpo1-1. In contrast to U1, U2, U4 and U5, the snRNA U6 gets a 5'- $\gamma$ monomethylcap instead of a $5^{\prime}-\mathrm{m}^{7} \mathrm{G}$ cap (Reddy et al., 1987; Singh and Reddy, 1989). The yeast Sm-class snRNAs may be exported via Xpo1 through the interaction with the CBC and 
Mex67 (Figure 11, Figure 12, Figure 29). Solely, U6 interacts with only Mex67 and not with Xpo1, which might be due to its different type of 5'-cap. Moreover, the current model is anyway that U6 does not shuttle to the cytoplasm, as shuttling has never been observed before (Sloan et al., 2016). However, it has been already shown for yeast cells that U6 mislocalizes to the cytoplasm in Lsm-ring mutants (Spiller et al., 2007). The importin $\beta$ mutant $r s l 1-1$ shows a cytoplasmic localization of both Lsm8 and U6. The question whether this mislocalization is generated by the missing nuclear retention of U6 through Lsm-ring interaction or by the disrupted Lsm-ring-dependent import still needs to be clarified (Spiller et al., 2007). In our study, we did not use rsl1-1 but the importin $\beta$ mutant kap95 E126K. Here, we did not see any accumulation of U6 in the cytoplasm. It will be interesting to repeat the FISH analysis and the cell fractionation experiments with different $r s l l$ mutants to further investigate the relevance of importin $\beta$ on U6 nuclear import.

To further investigate the hypothesis that Xpo1 exports the Sm-class snRNAs via the CBC, we performed co-immunoprecipitation experiments with Xpo1 and checked for its interaction with the CBC component Cbp80. We were able to show an interaction of Xpo1 with $\mathrm{Cbp} 80$ in vivo (Figure 29), suggesting that also in yeast snRNA export is mediated by Xpo1 via the CBC. In human, a special adaptor termed PHAX evolved (Ohno et al., 2000), which has no homologue in yeast (Matera et al., 2007). The question if Xpo1 contacts the CBC directly, needs further analysis, e.g. in vitro binding studies. Moreover, it is also possible that a currently unknown adaptor protein exists in yeast.

To further support the idea, that Mex67 and Xpo1 are exporting the snRNAs, we performed RNA co-immunoprecipitation experiments with GFP tagged proteins. In fact, we could detect a strong physical interaction of the snRNAs with both Xpo1 and Mex67 (Figure 12). The amount of bound RNA is comparable to the amount of precipitated TLC1 RNA in our previous study (Wu et al., 2014). In the next step, we investigated if Mex67 is able to bind the snRNAs directly. In vitro binding studies performed by Dr. Lysann Bender (Zander et al., 2016) showed a significant reduction of the interaction between RNA-binding defective mutants of Mex67 and the snRNAs (Figure 13). Whether human Tap-p15 can also bind to snRNAs directly is unknown, but it can bind to RNA in general (Köhler and Hurt, 2007). Even though some experiments with mutants of Tap-p15 led to mRNA export defects in human cells and did not affect the export of U5 (Braun et al., 2002), further detailed studies will be necessary to uncover a potential function of Tap-p15 in snRNA export. 


\subsection{Nuclear import of snRNAs is facilitated by Cse1 and Mtr10 in an Sm- ring-dependent manner}

Once snRNAs are exported to the cytoplasm, they have to be re-imported into the nucleus to fulfill their role in splicing. We screened mutants of all 14 yeast karyopherins for possible snRNA import defects (Figure 14). We identified Mtr10 and Cse1 to display clear import defects for the tested snRNAs (Figure 15). To support this finding, we isolated RNA from cytoplasmic fractions of mtrl04 and csel-1 cells and measured the amount of snRNAs compared to wild type (Figure 16). Consistent with the previous finding, the snRNAs were significantly enriched in the cytoplasm of mtrl04 and csel-1 cells. To show a direct effect of the two karyopherins on snRNA localization, we studied the physical interaction of Mtr10 and Cse1 with the snRNAs. The measured interactions between the importins and the snRNAs appeared with a high variance, especially for Cse1. A possible explanation for this could be the low stability of import complexes due to the presence of free Ran-GTP in the cell lysate, which might destabilize the karyopherin-substrate interactions. Nevertheless, there was a significant enrichment of interaction compared to the no tag control (Figure 17). For U6 only a rather weak interaction with Cse1 was measured, indicating a different mode of action. As Cse1 interacts with the Sm-ring (Figure 23), and U6 associates with an Lsm-ring (Achsel et al., 1999; Wilusz and Wilusz, 2013), there might be different importins for U6 than for the Sm-class snRNAs. This seems quite likely, as the deletion of the Lsm-ring results in a cytoplasmic accumulation of U6 but not in the mislocalization of U1 and U4 (Spiller et al., 2007).

Interestingly, Cse1 was shown to bind together with Ran-GTP to importin $\alpha$ in the nucleus and to translocate the NLS-adaptor to the cytoplasm (Hood and Silver, 1998). Importantly, to date, this is the only function described for Cse1. The protein consists of consecutive HEAT

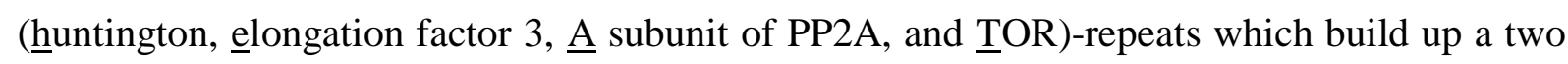
arch structure (Cook et al., 2005). Cse1 has a closed conformation in its cargo free state. In the presence of Ran-GTP the closed conformation opens and Ran-GTP binds to Cse1 and subsequently importin $\alpha$ can bind (Cook et al., 2005). It would be beneficial to investigate, if the Sm-proteins can also interrupt the closed conformation and form a stable import complex with Cse1, which is released in the nucleus by the association of Ran-GTP. Here, SmB is of special interest, because we could show a physical interaction between SmB and Cse1 (Figure 23). 
In order to examine the possibility of a direct protein-RNA interaction between Cse 1 and the snRNAs, we purified recombinant GST-Cse1 (Figure 21) and performed in vitro RNA-coimmunoprecipitation experiments. We were only able to purify small amounts of recombinant protein, as most of the protein did not bind to the column (Figure 21A). It was ,however, enough to perform the in vitro binding studies. Cse1 did not bind to the snRNAs in vitro (Figure 22). The result provides support for the idea that the interaction of Cse1 with the snRNAs might be mediated by the Sm-ring and the snRNAs are imported as an snRNP. The requirement of a complete snRNP assembly might represent an elegant quality assurance step, as incomplete snRNPs would not be imported. Furthermore, snRNAs without an Sm-ring are rapidly degraded by Dcp2 and Xrn1 (Shukla and Parker, 2014).

The second karyopherin we identified is Mtr10, a well characterized importin. It has been shown earlier that Mtr10 imports several substrates, e.g. the RNA binding protein Np13, Gbp2 and Hrb1 (Häcker and Krebber, 2004; Senger et al., 1998; Windgassen et al., 2004). It is also essential for the import of the non-coding RNA TLC1 and tRNA (Gallardo et al., 2008; Huang and Hopper, 2015). Therefore, it seems likely that Mtr10 might also be responsible for the snRNA import. How the import is facilitated is, however, still unknown. Mtr10 is a member of the importin $\beta$ family, which shares a common HEAT-repeat feature (Ström and Weis, 2001). The HEAT repeats build up a hydrophobic core for protein-protein interaction (Andrade et al., 2001). Hence, it is rather unlikely that they can directly bind to RNA, which could be investigated by in vitro binding studies. Due to structural similarities between Cse1 and Mtr10 it seems conceivable that Mtr10 may also need an adaptor to interact with the snRNAs. We performed co-immunoprecipitation experiments with Mtr10 and the Sm-ring members $\mathrm{SmB}$ and $\mathrm{SmG}$, but could not detect any interaction (data not shown). Mtr10 releases Npl3 from translating mRNAs (Windgassen et al., 2004). Hence, it could be possible that Mtr10 binds to Npl3 in the cytoplasm and Npl3 serves as an adaptor protein for the interaction of Mtr10 with the snRNAs. To test this hypothesis one might investigate the localization of snRNAs in $n p l 3 \Delta$ and determine, if the physical interaction of Mtr10 with the snRNAs is reduced in the absence of Npl3. This could be a possible import mechanism for other Mtr10 import substrates like TLC1. In addition, Transportin SR, the human homologue of Mtr10, is the importer for the human homologues of the yeast SR-proteins (Huang and Steitz, 2005). It would be interesting to investigate the role of Transportin SR in human snRNA and in non-coding RNA transport in general, because large RNPs most likely require more than one transport receptor. 


\section{3 snRNP assembly occurs in the cytoplasm and is essential for subsequent snRNA import}

One major goal of this project was the verification of the place of snRNP assembly. Therefore, we trapped the snRNAs in the nuclei of the three export mutant strains xpol-1, mex67-5 and the double mutant mex67-5 xpo1-1 and performed RNA-co-immunoprecipitation experiments with $\mathrm{SmB}$ in each strain. Upon snRNA nuclear trapping, the interaction of SmB with the Sm-class snRNAs U1 and U2 was significantly reduced, indicating that the interaction of protein and RNA cannot occur if the snRNAs do not shuttle through the cytoplasm (Figure 24). Interestingly, the Sm-ring members SmB and SmG do not mislocalize in the cytoplasm of mex67-5 cells, suggesting that the nuclear import of the Sm-proteins is independent of the snRNAs (Figure 19). Furthermore, SmB and both U1 and U2 did not interact in the nuclei of mex67-5 cells with each other, indicating that a potential assembly factor might be required that is localized in the cytoplasm. In human, the SMN-complex is needed for the assembly of the Sm-ring onto the snRNAs (Matera and Wang, 2014). To date, a potential yeast homolog has not been identified or if another, currently unknown factor that supports SM-ring loading exists. On the other hand, it is also possible that the Sm-proteins are imported into nucleus as a partially assembled ring, which cannot finalize its ring structure in the cytoplasm, because the Sm-ring assembly is RNA dependent as recently shown ( $\mathrm{Li}$ et al., 2016). Once the Sm-proteins are in the nucleus, the interaction of the snRNAs and the Smproteins might still be prevented, because the snRNAs are already recruited by the spliceosomes (Figure 32 and Figure 33) and therefore not accessible for the Sm-ring anymore.

To address the question, if the Sm-ring assembly is important for snRNA import, we localized the Sm-ring members SmB and SmG in the snRNA import mutants cse 1-1 and mtr104 (Figure 18). Both SmB and SmG mislocalized in the cytoplasm of both strains. Additionally, we used an Sm-ring double mutant, in which the depletion of SmB in combination with a Cterminal truncated version of SmD1 not only results in the accumulation of both the Smproteins in the cytoplasm (Bordonné, 2000) but also of the snRNAs as shown here (Figure 20).

Taken together, the following facts support the idea that snRNAs undergo nucleo-cytoplasmic shuttling in yeast: (i) The Sm-proteins and the snRNAs accumulate in cse1-1 and mtr104 (Figure 15 and Figure 18), (ii) the double Sm-protein mutant results in a cytoplasmic accumulation of the snRNAs (Figure 20), and (iii) the physical interaction of SmB and the 
snRNAs is significantly reduced in the export mutants mex67-5, xpo1-1 and the double mutant mex67-5 xpo1-1 (Figure 24).

\subsection{3'-processing of snRNAs takes place in the nucleus after nucleo- cytoplasmic shuttling and nucleolar TMG-capping finalizes snRNA processing by the inhibition of ongoing snRNP export}

In human cells, 3'- processing of snRNAs takes place in the cytoplasm by an unknown endoribonuclease (Matera and Wang, 2014). The processing of yeast snRNAs was shown to occur in the nucleus (Catala et al., 2004; Seipelt et al., 1999). This is consistent with our results, as the retention of the snRNAs in the cytoplasm in the import mutants cse 1-1 and mtr104 results in defective U1 processing (Figure 25). Notably, the snRNA export block in mex67-5 xpo1-1 results in the same effect. This is reasonable because the Sm-ring is essential for the snRNA processing (Coy et al., 2013; Seipelt et al., 1999) and our results indicate that the Sm-ring assembles on the snRNAs after nuclear export (Figure 24). Hence, we propose that the yeast U1 snRNA is processed after nucleo-cytoplasmic shuttling.

One step in the maturation of human snRNAs, which is essential for their import, is the cytoplasmic trimethylation of the $5^{\prime}-\mathrm{m}^{7} \mathrm{G}$ cap by TGS1 (Fischer and Lührmann, 1990; Matera and Wang, 2014). Trimethylation enables binding of SPN and subsequently of importin $\beta$ (Mouaikel et al., 2003). In yeast, Tgs1 is located in the nucleolus (Mouaikel et al., 2002). We determined the time point of trimethylation of yeast snRNAs by Tgs1. If the snRNAs are trimethylated after the nucleo-cytoplasmic shuttling, it would support our idea that Xpo1 facilitates the export of snRNAs via the $\mathrm{CBC}$ because the $\mathrm{m}^{7} \mathrm{G}$-cap promotes $\mathrm{CBC}$ recruitment to snRNAs (Schwer et al., 2011). Thus, we performed RNA immunoprecipitations with a TMG-cap binding antibody in the import mutants $\operatorname{cse} 1-1$ and mtr104. As the antibody is not completely specific for the TMG-cap, but also has a low affinity for $\mathrm{m}^{7} \mathrm{G}$-caps, we used the $\operatorname{tg} \mathrm{s} 1 \Delta$ strain to determine the level of unspecific binding of $\mathrm{m}^{7} \mathrm{G}$-capped snRNAs. Indeed, the amount of immunoprecipitated RNA in cse $1-1$ and mtr10 is comparable to that in $\operatorname{tgs} 1 \Delta$ (Figure 26). Both $\mathrm{U} 1$ and $\mathrm{U} 2$, show significantly reduced levels in both import mutants compared to wild type, indicating that trimethylation takes place after nucleo-cytoplasmic shuttling. This is further supported by our finding that the Sm-ring assembles on the snRNAs in the cytoplasm and by the fact that the interaction of $\mathrm{SmB}$ with the snRNA is not affected in tgs $1 \Delta$ (Schwer et al., 2011). Previous studies revealed an in vitro but not in vivo interaction of both $\mathrm{SmB}$ and $\mathrm{SmD} 1$ with $\mathrm{Tgs} 1$ as this interaction in vivo might be rather transient and therefore not detectable (Mouaikel et al., 2002). In contrast, we were 
able to show the physical interaction between $\mathrm{SmB}$ and Tgs1 in vivo (Figure 28). Furthermore, we visualized U1 and U2 in cells that were depleted for SMB1 and localized the snRNA in the nucleolus (Figure 27). This supports the idea that $\mathrm{SmB}$ is needed for the trimethylation of snRNAs by Tgs1. The necessity of the Sm-ring for trimethylation has already been proposed in previous studies as depletion of $\mathrm{SmD} 1$ or $\mathrm{SmD} 3$ resulted in the loss of snRNA trimethylation (Roy et al., 1995; Rymond, 1993).

The tgs $1 \Delta$ strain displays growth defects at low temperatures $\left(16^{\circ} \mathrm{C}\right)($ Schwer et al., 2011). It has been shown that this cold sensitivity can be suppressed by a point mutation in the RNA binding domain of Cbp20. Therefore, Schwer and collegues suggested that the growth defect is not caused by the missing TMG-cap itself but might be induced by the enhanced binding of the CBC to $\mathrm{m}^{7} \mathrm{G}$-capped snRNAs. Strikingly, the composition of the U1 snRNP is not altered through the enhanced CBC binding to snRNAs (Schwer et al., 2011). We have shown that trimethylation is performed after snRNP assembly and it is known that the access of CBC to TMG-capped snRNAs is much lower than to $\mathrm{m}^{7} \mathrm{G}$-capped snRNAs (Schwer et al., 2011). This is also confirmed by our finding that TMG-capped snRNAs preferentially bind to late splicing factors instead of Mex67 and Xpo1 (Fig. 30). Therefore, we propose a model where the trimethylation of the snRNAs is the final step in the snRNA maturation process. The addition of the TMG-cap blocks further Xpo1 access and thus, inhibits the ongoing export of the mature snRNP.

\subsection{Nucleo-cytoplasmic shuttling is essential for correct spliceosome assembly and a fully functional splicing machinery}

We could already show that Mex67 can bind to snRNAs in vivo and in vitro (Figure 12 and Figure 13). This binding may be needed to directly export the snRNAs out of the nucleus, which would prohibit the integration of faulty snRNAs into the spliceosome, as shown to be the case for the export mutant mex67-5. The accumulation of unprocessed $\mathrm{U} 1$ in the nucleus results in the integration of the U1 precursor into the spliceosomes (Figure 32 and Figure 33).

Based on these results, we examined general consequences of the integration of aberantly processed snRNAs into spliceosomes. Therefore, we first depleted the snRNAs from the nucleus by trapping them in the cytoplasm of mtr10 4 and cse $1-1$ cells. We calculated the ratio of intron containing transcripts compared to the total amount of the corresponding transcripts of four different genes (EFB1, RPS23A, RPL21B and RPS6A). Indeed, we revealed a significant enrichment of RNAs that contain intronic regions in the pool of total RNAs (Figure 31), illustrating that the depletion of snRNAs from the nucleus is deleterious for 
splicing. Furthermore, we wanted to provide insight into the consequences of the implementation of faulty snRNAs into spliceosomes. Therefore, we calculated the enrichment of intron containing transcripts compared to total RNA in xpo1-1, mex67-5 and the double mutant mex67-5 xpo1-1 (Figure 35). Once again, we revealed severe splicing defects for the selected transcripts. Finally, we performed a genome wide RNA-Sequencing study to receive an overview over the whole transcriptome. Strikingly, $\sim 80 \%$ of all detected intron containing transcripts (144 in total) were significantly enriched in the experiments compared to wild type (Figure 36B). We observed an average enrichment of around $\sim 4.5$ fold (Figure 36A). The splicing defects are widespread over several functional groups (Figure 37), indicating general splicing defects.

\subsection{Nucleo-cytoplasmic shuttling represents an elegant quality assurance mechanism for snRNP assembly}

The export of snRNAs could be a part of a quality control mechanism by rapidly depleting unprocessed U1 from the nucleus. Previously, it has been questioned why cells export snRNAs into the cytoplasm only for their re-import as snRNPs. Matera and Wang proposed that the export of snRNAs enables a possibility to prevent access of partially assembled snRNPs to their substrates (Matera and Wang, 2014). However, to date, this hypothesis has not been proven. Here, we show for the first time that unprocessed U1 can be integrated into spliceosomes if snRNA export is blocked (Fig. 32 and Fig. 33). Furthermore, we demonstrate splicing defects in all transport mutant strains (Figs. 31, 35, 36, 37). To further address the question if spliceosomes can distinguish between mature and immature snRNAs one could overexpress an Rnt1-cleavage incompetent U1 mutant in an SNR19 deletion strain. This would allow excluding the possibility of splicing defects generated from secondary effects in the RNA export mutant mex67-5 xpo1-1. Alternatively, cells with temporarily downregulated $R N T 1$ could be used to address this question.

Although, we cannot completely exclude secondary effects, our results are in agreement with other studies concerning the assembly of faulty snRNPs. In the study of Zhang and colleagues, the incorrect assembly of snRNPs results in wide spread splicing defects in all tested tissues and mice cells. Similar to our results, the examination of functional groups could not reveal any specific functional groups suffering from enriched splicing defects (Zhang et al., 2008). This strengthens our hypothesis that incorrect snRNPs induce splicing defects. 


\subsection{Novel model of snRNP maturation in S. cerevisiae}

Based on the findings of this PhD-project, we propose the following model for the snRNP maturation in S. cerevisiae (Figure 38). The snRNAs are transcribed by RNAP II and subsequently the pre-snRNAs are exported on a fast track out of the nucleus by Mex67 and Xpo1 to prevent the integration of pre-snRNAs into the spliceosome. Xpo1 interacts with CBC, which binds the $5^{\prime}-\mathrm{m}^{7} \mathrm{G}$-cap of the snRNAs (Schwer et al., 2011). Once the snRNAs reached the cytoplasm, the Sm-ring assembles on the snRNAs and the snRNP is imported into the nucleus. The import is facilitated by the karyopherins Cse1 and Mtr10. Cse1 binds the snRNAs via the Sm-ring. Thus, we propose that the Sm-ring dependent binding of the importins is the first quality assurance step of snRNP maturation. snRNAs, which are not associated to the Sm-ring, are degraded in the cytoplasm by Dcp2 and Xrn1 (Shukla and Parker, 2014). After import, snRNAs are 3'-processed by Rnt1 and Rrp6 (Coy et al., 2013; Seipelt et al., 1999; Shukla and Parker, 2014). The final step in snRNP maturation is performed by Tgs1, which trimethylates the $\mathrm{m}^{7} \mathrm{G}$-cap of the snRNAs (Mouaikel et al., 2002) and thereby inhibits the $\mathrm{CBC}$ from binding and in that way prevents the repeated export of the snRNAs via Xpo1. TMG-capping resembles another quality control step because the trimethylation is only possible if the Sm-ring is correctly associated to the snRNA. Finally, the snRNP is integrated into the spliceosome. Upon blocking of snRNA export, precursor snRNAs are recruited to spliceosomes resulting in genome wide splicing defects.

Our study provides insights into the quality control mechanisms of snRNP assembly and the physiological relevance of such a mechanism. Our model explains why a shuttling-dependent assembly mechanism potentially evolved during evolution.

We answered the long standing question, if snRNAs undergo a nucleo-cytoplasmic shuttling in yeast. In addition, we identified the mode of export, the cytoplasmic snRNP assembly, the snRNP import and the final nuclear snRNA processing steps, which uncovers similarities and differences to the human system. Importantly, it shows that snRNA shuttling is a common theme and necessary for the generation of functional spliceosomes and splicing. 


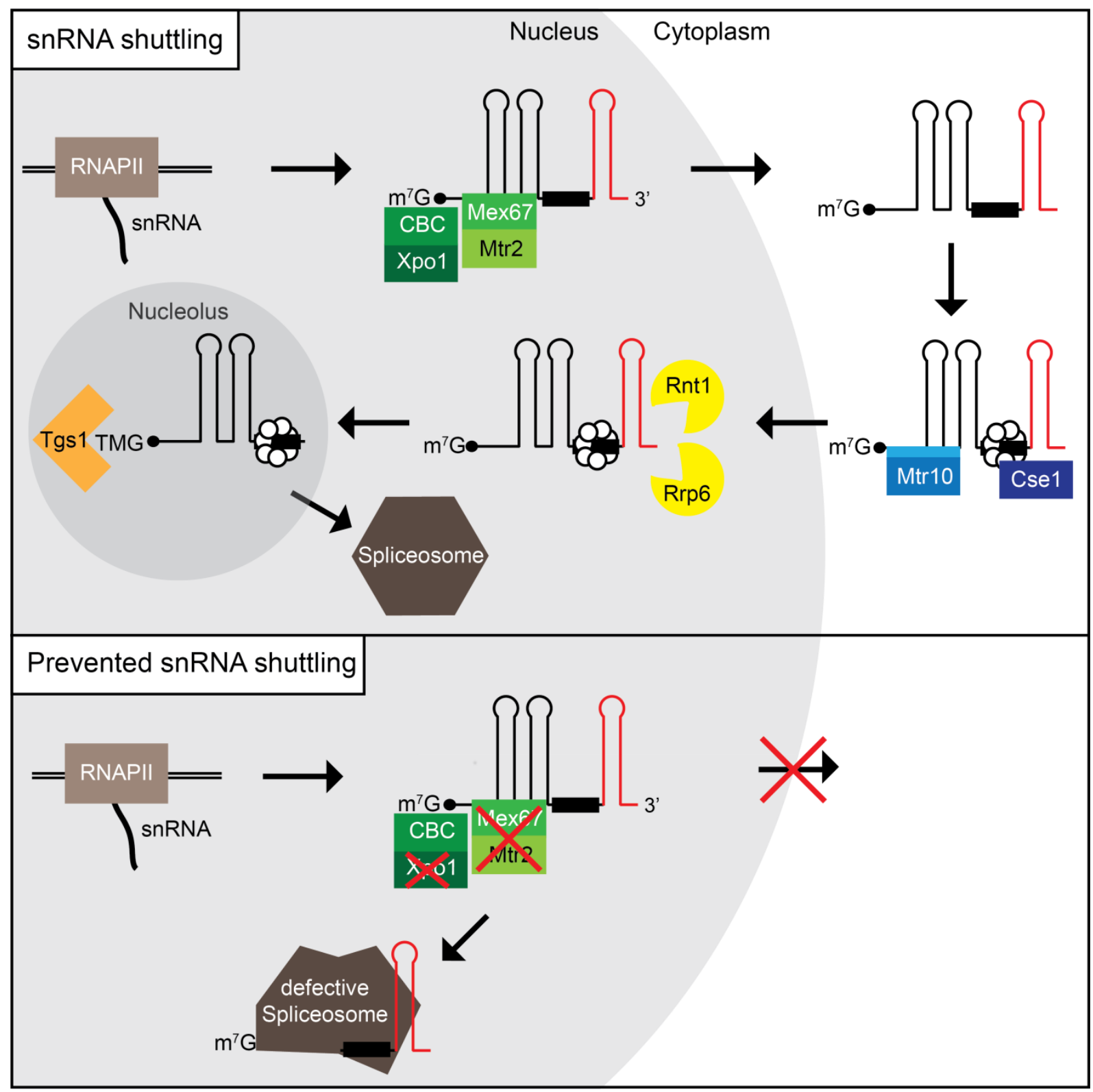

Figure 38: Model for snRNA transport and snRNP maturation in S. cerevisiae. Top: After RNAP II transcription, pre-snRNAs are exported to the cytoplasm by Mex67 and Xpo1, the latter via the interaction with the CBC. In the cytoplasm, both export receptors dissociate from the pre-snRNA and the Sm-ring assembles on the pre-snRNA. Afterwards, the pre-snRNP is imported to the nucleus via Mtr10 and Cse1, which interacts with the Sm-ring. Thereafter, the pre-snRNA is 3'-processed by the endoribonuclease Rnt1 and the exoribunuclease Rrp6 up to the Sm-ring binding site. Finally, the snRNA is trimethylated by Tgs 1 in the nucleolus. Bottom: The inhibition of pre-snRNA export from the nucleus in the RNA export mutant mex67-5 xpo1-1 results in the integration of pre-snRNAs into the spliceosome and leads to severe genome wide splicing defects. 


\section{References}

Achsel, T., Brahms, H., Kastner, B., Bachi, A., Wilm, M., and Lührmann, R. (1999). A doughnut-shaped heteromer of human Sm-like proteins binds to the 3'-end of U6 snRNA, thereby facilitating U4/U6 duplex formation in vitro. EMBO J. 18, 5789-5802.

Ahmad, S., Bhatia, K., Kannan, A., and Gangwani, L. (2016). Molecular Mechanisms of Neurodegeneration in Spinal Muscular Atrophy. J. Exp. Neurosci. 10, 39-49.

Aitchison, J.D., and Rout, M.P. (2012). The yeast nuclear pore complex and transport through it. Genetics 190, 855-883.

Akhtar, N., Hagan, H., Lopilato, J.E., and Corbett, A.H. (2001). Functional analysis of the yeast Ran exchange factor Prp20p: In vivo evidence for the RanGTP gradient model. Mol. Genet. Genomics 265, 851-864.

Alegria-Schaffer, A. (2014). Western blotting using chemiluminescent substrates. Methods Enzymol. 541, 251-259.

Allmang, C., Kufel, J., Chanfreau, G., Mitchell, P., Petfalski, E., and Tollervey, D. (1999). Functions of the exosome in rRNA, snoRNA and snRNA synthesis. EMBO J. 18, 5399-5410. Andrade, M.A., Petosa, C., O’Donoghue, S.I., Müller, C.W., and Bork, P. (2001). Comparison of ARM and HEAT protein repeats. J. Mol. Biol. 309, 1-18.

Baejen, C., Torkler, P., Gressel, S., Essig, K., Söding, J., and Cramer, P. (2014). Transcriptome Maps of mRNP Biogenesis Factors Define Pre-mRNA Recognition. Mol. Cell $55,745-757$.

Baillat, D., Hakimi, M.A., Näär, A.M., Shilatifard, A., Cooch, N., and Shiekhattar, R. (2005). Integrator, a multiprotein mediator of small nuclear RNA processing, associates with the Cterminal repeat of RNA polymerase II. Cell 123, 265-276.

Battle, D.J., Lau, C.K., Wan, L., Deng, H., Lotti, F., and Dreyfuss, G. (2006). The Gemin5 Protein of the SMN Complex Identifies snRNAs. Mol. Cell 23, 273-279.

Björklund, S., and Gustafsson, C.M. (2005). The yeast Mediator complex and its regulation. Trends Biochem. Sci. 30, 240-244.

Boda, B., Mas, C., Giudicelli, C., Nepote, V., Guimiot, F., Levacher, B., Zvara, A., Santha, M., LeGall, I., and Simonneau, M. (2004). Survival motor neuron SMN1 and SMN2 gene 
promoters: identical sequences and differential expression in neurons and non-neuronal cells. Eur. J. Hum. Genet. 12, 729-737.

Boon, K.-L., Pearson, M.D., and Koš, M. (2015). Self-association of Trimethylguanosine Synthase Tgs1 is required for efficient snRNA/snoRNA trimethylation and pre-rRNA processing. Sci. Rep. 5, 11282.

Bordonné, R. (2000). Functional characterization of nuclear localization signals in yeast Sm proteins. Mol. Cell. Biol. 20, 7943-7954.

Braun, I.C., Herold, A., Rode, M., and Izaurralde, E. (2002). Nuclear export of mRNA by TAP/NXF1 requires two nucleoporin-binding sites but not p15. Mol. Cell. Biol. 22, 54055418.

Brow, D.A., and Guthrie, C. (1990). Transcription of a yeast U6 snRNA gene requires a polymerase III promoter element in a novel position. Genes Dev. 4, 1345-1356.

Brune, C., Munchel, S.E., Fischer, N., Podtelejnikov, A. V, and Weis, K. (2005). Yeast poly ( A ) -binding protein Pab1 shuttles between the nucleus and the cytoplasm and functions in mRNA export. Rna-a Publ. Rna Soc. 517-531.

Camasses, a, Bragado-Nilsson, E., Martin, R., Séraphin, B., and Bordonné, R. (1998). Interactions within the yeast Sm core complex: from proteins to amino acids. Mol. Cell. Biol. $18,1956-1966$.

Carroll, K.L., Ghirlando, R., Ames, J.M., and Corden, J.L. (2007). Interaction of yeast RNAbinding proteins Nrd1 and Nab3 with RNA polymerase II terminator elements. RNA 13, 361373.

Catala, M., Lamontagne, B., Larose, S., Ghazal, G., and Elela, S.A. (2004). Cell cycledependent nuclear localization of yeast RNase III is required for efficient cell division. Mol. Biol. Cell 15, 3015-3030.

Chanfreau, G., Elela, S.A., Ares, M., and Guthrie, C. (1997). Alternative 3'-end processing of U5 snRNA by RNase III. Genes Dev. 11, 2741-2751.

Cho, E.J., Takagi, T., Moore, C.R., and Buratowski, S. (1997). mRNA capping enzyme is recruited to the transcription complex by phosphorylation of the RNA polymerase II carboxyterminal domain. Genes Dev. 11, 3319-3326.

Colau, G., Thiry, M., Leduc, V., Bordonné, R., and Lafontaine, D.L.J. (2004). The small 
nucle(ol)ar RNA cap trimethyltransferase is required for ribosome synthesis and intact nucleolar morphology. Mol. Cell. Biol. 24, 7976-7986.

Conti, E., Uy, M., Leighton, L., Blobel, G., and Kuriyan, J. (1998). Crystallographic analysis of the recognition of a nuclear localization signal by the nuclear import factor karyopherin $\alpha$. Cell 94, 193-204.

Cook, A., Fernandez, E., Lindner, D., Ebert, J., Schlenstedt, G., and Conti, E. (2005). The structure of the nuclear export receptor Cse1 in its cytosolic state reveals a closed conformation incompatible with cargo binding. Mol. Cell 18, 355-367.

Coppola, J.A., Field, A.S., and Luse, D.S. (1983). Promoter-proximal pausing by RNA polymerase II in vitro: transcripts shorter than 20 nucleotides are not capped. Proc. Natl. Acad. Sci. U. S. A. 80, 1251-1255.

Coy, S., Volanakis, A., Shah, S., and Vasiljeva, L. (2013). The Sm Complex Is Required for the Processing of Non-Coding RNAs by the Exosome. PLoS One 8.

Cramer, P., Armache, K.-J.J., Baumli, S., Benkert, S., Brueckner, F., Buchen, C., Damsma, G.E., Dengl, S., Geiger, S.R., Jasiak, A.J., et al. (2008). Structure of eukaryotic RNA polymerases. Annu Rev Biophys 37, 337-352.

Creamer, T.J., Darby, M.M., Jamonnak, N., Schaughency, P., Hao, H., Wheelan, S.J., and Corden, J.L. (2011). Transcriptome-wide binding sites for components of the Saccharomyces cerevisiae non-poly(A) termination pathway: Nrd1, Nab3, and Sen1. PLoS Genet. 7.

Dingwall, C., and Laskey, R.A. (1991). Nuclear targeting sequences--a consensus? Trends Biochem. Sci. 16, 478-481.

Dobin, A., Davis, C.A., Schlesinger, F., Drenkow, J., Zaleski, C., Jha, S., Batut, P., Chaisson, M., and Gingeras, T.R. (2013). STAR: Ultrafast universal RNA-seq aligner. Bioinformatics $29,15-21$.

Egloff, S. (2012). Role of Ser7 phosphorylation of the CTD during transcription of snRNA genes. RNA Biol. 9, 1033-1038.

Egloff, S., O’Reilly, D., Chapman, R.D., Taylor, A., Tanzhaus, K., Pitts, L., Eick, D., and Murphy, S. (2007). Serine-7 of the RNA polymerase II CTD is specifically required for snRNA gene expression. Science 318, 1777-1779.

Egloff, S., Al-Rawaf, H., O'Reilly, D., and Murphy, S. (2009). Chromatin structure is 
implicated in "late" elongation checkpoints on the U2 snRNA and beta-actin genes. Mol. Cell. Biol. 29, 4002-4013.

Elela, S.A., and Ares, M. (1998). Depletion of yeast RNase III blocks correct U2 3' end formation and results in polyadenylated but functional U2 snRNA. EMBO J. 17, 3738-3746.

Eschenlauer, J.B., Kaiser, M.W., Gerlach, V.L., and Brow, D.A. (1993). Architecture of a yeast U6 RNA gene promoter. Mol. Cell. Biol. 13, 3015-3026.

Fischer, U., and Lührmann, R. (1990). An Essential Signaling Role for the m3G Cap in the Transport of U1 snRNP to the Nucleus. Science (80-. ). 249, 786-790.

Fischer, U., Sumpter, V., Sekine, M., Satoh, T., and Luhrmann, R. (1993). Nucleocytoplasmic transport of U snRNPs: definition of a nuclear location signal in the Sm core domain that binds a transport receptor independently of the m3G cap. Embo J 12, 573-583.

Fleischmann, M., Clark, M.W., Forrester, W., Wickens, M., Nishimoto, T., and Aebi, M. (1991). Analysis of yeast prp20 mutations and functional complementation by the human homologue RCC1, a protein involved in the control of chromosome condensation. MGG Mol. Gen. Genet. 227, 417-423.

Folkmann, A.W., Noble, K.N., Cole, C.N., and Wente, S.R. (2011). Dbp5, Gle1-IP6, and Nup159: A working model for mRNP export. Nucleus 2, 540-548.

Fornerod, M., Ohno, M., Yoshida, M., and Mattaj, I.W. (1997). CRM1 is an export receptor for leucine-rich nuclear export signals. Cell 90, 1051-1060.

Fried, H., and Kutay, U. (2003). Nucleocytoplasmic transport: Taking an inventory. Cell. Mol. Life Sci. 60, 1659-1688.

Gallardo, F., Olivier, C., Dandjinou, A.T., Wellinger, R.J., and Chartrand, P. (2008). TLC1 RNA nucleo-cytoplasmic trafficking links telomerase biogenesis to its recruitment to telomeres. EMBO J. 27, 748-757.

Garfin, D.E. (2009). One-Dimensional Gel Electrophoresis1. Methods Enzymol. 463, 497513.

Ghaemmaghami, S., Huh, W.-K., Bower, K., Howson, R.W., Belle, A., Dephoure, N., O'Shea, E.K., and Weissman, J.S. (2003). Global analysis of protein expression in yeast. Nature 425, 737-741. 
Gibson, D.G. (2011). Enzymatic assembly of overlapping DNA fragments. Methods Enzymol. 498, 349-361.

Gibson, D.G., Young, L., Chuang, R.Y., Venter, J.C., Hutchison, C.A., and Smith, H.O. (2009). Enzymatic assembly of DNA molecules up to several hundred kilobases. Nat. Methods 6, 343-345.

Gietz, D., St Jean, A., Woods, R. a, and Schiestl, R.H. (1992). Improved method for high efficiency transformation of intact yeast cells. Nucl Acid Res 20, 1425.

Görlich, D., Henklein, P., Laskey, R. a, and Hartmann, E. (1996). A 41 amino acid motif in importin-alpha confers binding to importin-beta and hence transit into the nucleus. EMBO J. $15,1810-1817$.

Gorsch, L., Dockendorff, T.C., and Cole, C.N. (1995). A Conditional Allele of the Novel Repeat-containing Yeast Nucleoporin. Jcb 129, 939-955.

Gottschalk, A., Tang, J., Puig, O., Salgado, J., Neubauer, G., Colot, H. V, Mann, M., Seraphin, B., Rosbash, M., Lührmann, R., et al. (1998). A comprehensive biochemical and genetic analysis of the yeast U1 snRNP reveals five novel proteins. Rna 4, 374-393.

Gu, M., Rajashankar, K.R., and Lima, C.D. (2010). Structure of the Saccharomyces cerevisiae Cet1-Ceg1 mRNA Capping Apparatus. Structure 18, 216-227.

Häcker, S., and Krebber, P. (2004). Differential Export Requirements for Shuttling Serine/Arginine-type mRNA-binding Proteins. J. Biol. Chem. 279, 5049-5052.

Hackmann, A., Wu, H., Schneider, U.-M., Meyer, K., Jung, K., and Krebber, H. (2014). Quality control of spliced mRNAs requires the shuttling SR proteins Gbp2 and Hrb1. Nat. Commun. 5, 1-14.

Hahn, S., and Schlenstedt, G. (2011). Importin beta-type nuclear transport receptors have distinct binding affinities for Ran-GTP. Biochem. Biophys. Res. Commun. 406, 383-388.

Hamm, J., and Mattaj, I.W. (1990). Monomethylated cap structures facilitate RNA export from the nucleus. Cell 63, 109-118.

Hardin, J.W., Warnasooriya, C., Kondo, Y., Nagai, K., and Rueda, D. (2015). Assembly and dynamics of the U4/U6 di-snRNP by single-molecule FRET. Nucleic Acids Res. 43, 1096310974. 
Henry, R.W., Mittal, V., Ma, B., Kobayashi, R., and Hernandez, N. (1998). SNAP19 mediates the assembly of a functional core promoter complex (SNAP(c)) shared by RNA polymerases II and III. Genes Dev. 12, 2664-2672.

Heo, D.H., Yoo, I., Kong, J., Lidschreiber, M., Mayer, A., Choi, B.Y., Hahn, Y., Cramer, P., Buratowski, S., and Kim, M. (2013). The RNA polymerase 2 C-terminal domain-interacting domain of yeast Nrd1 contributes to the choice of termination pathway and couples to RNA processing by the nuclear exosome. J. Biol. Chem. 288, 36676-36690.

Hermann, H., Fabrizio, P., Raker, V.A., Foulaki, K., Hornig, H., Brahms, H., and Lührmann, R. (1995). snRNP Sm proteins share two evolutionarily conserved sequence motifs which are involved in Sm protein-protein interactions. EMBO J. 14, 2076-2088.

Holmes, R.K., Tuck, A.C., Zhu, C., Dunn-Davies, H.R., Kudla, G., Clauder-Munster, S., Granneman, S., Steinmetz, L.M., Guthrie, C., and Tollervey, D. (2015). Loss of the Yeast SR Protein Np13 Alters Gene Expression Due to Transcription Readthrough. PLoS Genet. 11.

Hood, J.K., and Silver, P.A. (1998). Cse1p is required for export of Srp1p/importin- $\alpha$ from the nucleus in Saccharomyces cerevisiae. J. Biol. Chem. 273, 35142-35146.

van Hoof, A., Lennertz, P., and Parker, R. (2000). Yeast exosome mutants accumulate 3'extended polyadenylated forms of U4 small nuclear RNA and small nucleolar RNAs. Mol. Cell. Biol. 20, 441-452.

Hopper, A.K., Traglia, H.M., and Dunst, R.W. (1990). The yeast RNA1 gene product necessary for RNA processing is located in the cytosol and apparently excluded from the nucleus. J. Cell Biol. 111, 309-321.

Hsin, J., and Manley, J.L. (2012). The RNA polymerase II CTD coordinates transcription and RNA processing. Genes Dev. 2119-2137.

Huang, H.Y., and Hopper, A.K. (2015). In vivo biochemical analyses reveal distinct roles of beta-importins and eEF1A in tRNA subcellular traffic. Genes Dev. 29, 772-783.

Huang, Y., and Steitz, J.A. (2005). SRprises along a messenger's journey. Mol. Cell 17, 613615.

Huber, J., Cronshagen, U., Kadokura, M., Marshallsay, C., Wada, T., Sekine, M., and Lührmann, R. (1998). Snurportin1, an m3G-cap-specific nuclear import receptor with a novel domain structure. EMBO J. 17, 4114-4126. 
Hurt, E., Sträßer, K., Segref, A., Bailer, S., Schlaich, N., Presutti, C., Tollervey, D., and Jansen, R. (2000). Mex67p mediates nuclear export of a variety of RNA polymerase II transcripts. J. Biol. Chem. 275, 8361-8368.

Inoue, H., Nojima, H., and Okayama, H. (1990). High efficiency transformation of Escherichia coli with plasmids. Gene 96, 23-28.

Izaurralde, E., Lewis, J., Gamberi, C., Jarmolowski, A., McGuigan, C., and Mattaj, I.W. (1995). A cap-binding protein complex mediating U snRNA export. Nature 376, 709-712.

Jády, B.E., Darzacq, X., Tucker, K.E., Gregory Matera, A., Bertrand, E., and Kiss, T. (2003). Modification of Sm small nuclear RNAs occurs in the nucleoplasmic Cajal body following import from the cytoplasm. EMBO J. 22, 1878-1888.

Jeronimo, C., Forget, D., Bouchard, A., Li, Q., Chua, G., Poitras, C., Thérien, C., Bergeron, D., Bourassa, S., Greenblatt, J., et al. (2007). Systematic Analysis of the Protein Interaction Network for the Human Transcription Machinery Reveals the Identity of the 7SK Capping Enzyme. Mol. Cell 27, 262-274.

Jiao, X., Xiang, S., Oh, C., Martin, C.E., Tong, L., and Kiledjian, M. (2010). Identification of a quality-control mechanism for mRNA 5 '-end capping. Nature 467, 608-611.

Kambach, C., Walke, S., and Nagai, K. (1999). Structure and assembly of the spliceosomal small nuclear ribonucleoprotein particles. Curr. Opin. Struct. Biol. 9, 222-230.

Kelly, S.M., and Corbett, A.H. (2009). Messenger RNA export from the nucleus: A series of molecular wardrobe changes. Traffic 10, 1199-1208.

Kitao, S., Segref, A., Kast, J., Wilm, M., Mattaj, I.W., and Ohno, M. (2008). A compartmentalized phosphorylation/dephosphorylation system that regulates $U$ snRNA export from the nucleus. Mol. Cell. Biol. 28, 487-497.

Köhler, A., and Hurt, E. (2007). Exporting RNA from the nucleus to the cytoplasm. Nat. Rev. Mol. Cell Biol. 8, 761-773.

Kroiss, M., Schultz, J., Wiesner, J., Chari, A., Sickmann, A., and Fischer, U. (2008). Evolution of an RNP assembly system: a minimal SMN complex facilitates formation of UsnRNPs in Drosophila melanogaster. Proc. Natl. Acad. Sci. U. S. A. 105, 10045-10050.

Kubicek, K., Cerna, H., Holub, P., Pasulka, J., Hrossova, D., Loehr, F., Hofr, C., Vanacova, S., and Stefl, R. (2012). Serine phosphorylation and proline isomerization in RNAP II CTD 
control recruitment of Nrdl. Genes Dev. 26, 1891-1896.

Kunkel, G.R., Maser, R.L., Calvet, J.P., and Pederson, T. (1986). U6 small nuclear RNA is transcribed by RNA polymerase III. Proc. Natl. Acad. Sci. U. S. A. 83, 8575-8579.

Laemmli, U.K. (1970). Cleavage of structural proteins during the assembly of the head of bacteriophage T4. Nature 227, 680-685.

Lefebvre, S., Bürglen, L., Reboullet, S., Clermont, O., Burlet, P., Viollet, L., Benichou, B., Cruaud, C., Millasseau, P., Zeviani, M., et al. (1995). Identification and characterization of a spinal muscular atrophy-determining gene. Cell 80, 155-165.

Lefebvre, S., Burlet, P., Liu, Q., Bertrandy, S., Clermont, O., Munnich, a, Dreyfuss, G., and Melki, J. (1997). Correlation between severity and SMN protein level in spinal muscular atrophy. Nat. Genet. 16, 265-269.

Legrain, P., and Rosbash, M. (1989). Some cis- and trans-acting mutants for splicing target pre-mRNA to the cytoplasm. Cell 57, 573-583.

Lei, E.P., Krebber, H., and Silver, P.A. (2001). Messenger RNAs are recruited for nuclear export during transcription. Genes Dev. 15, 1771-1782.

Lewis, J.D., and Izaurralde, E. (1997). The Role of the Cap Structure in RNA Processing and Nuclear Export. Eur. J. Biochem. 247, 461-469.

Li, J., Leung, A.K., Kondo, Y., Oubridge, C., and Nagai, K. (2016). Re-refinement of the spliceosomal U4 snRNP core-domain structure. Acta Crystallogr. Sect. D Struct. Biol. 72, $131-146$.

Liu, Q., Fischer, U., Wang, F., and Dreyfuss, G. (1997). The spinal muscular atrophy disease gene product, SMN, and its associated protein SIP1 are in a complex with spliceosomal snRNP proteins. Cell 90, 1013-1021.

Liu, Y., Guo, W., Tartakoff, P.Y., and Tartakoff, a M. (1999). A Crm1p-independent nuclear export path for the mRNA-associated protein, Npl3p/Mtr13p. Proc. Natl. Acad. Sci. U. S. A. 96, 6739-6744.

Lobo, S.M., Tanaka, M., Sullivan, M.L., and Hernandez, N. (1992). A TBP complex essential for transcription from TATA-less but not TATA-containing RNA polymerase III promoters is part of the TFIIB fraction. Cell 71, 1029-1040. 
Loeb, J.D., Schlenstedt, G., Pellman, D., Kornitzer, D., Silver, P.A., and Fink, G.R. (1995). The yeast nuclear import receptor is required for mitosis. Proc. Natl. Acad. Sci. U. S. A. 92, $7647-7651$.

Margottin, F., Dujardin, G., Gérard, M., Egly, J.M., Huet, J., and Sentenac, A. (1991). Participation of the TATA factor in transcription of the yeast U6 gene by RNA polymerase C. Sci. (New York, NY) 251, 424-426.

Matera, A.G., and Wang, Z. (2014). A day in the life of the spliceosome. Nat. Rev. Mol. Cell Biol. 15, 108-121.

Matera, A.G., Terns, R.M., and Terns, M.P. (2007). Non-coding RNAs: lessons from the small nuclear and small nucleolar RNAs. Nat Rev Mol Cell Biol 8, 209-220.

Mattaj, I.W., Dathan, N.A., Parry, H.D., Carbon, P., and Krol, A. (1988). Changing the RNA polymerase specificity of U snRNA gene promoters. Cell 55, 435-442.

McCracken, S., Fong, N., Rosonina, E., Yankulov, K., Brothers, G., Siderovski, D., Hessel, A., Foster, S., Shuman, S., and Bentley, D.L. (1997). 5'-Capping enzymes are targeted to premRNA by binding to the phosphorylated carboxy-terminal domain of RNA polymerase II. Genes Dev. 11, 3306-3318.

Meister, G., Eggert, C., Bühler, D., Brahms, H., Kambach, C., and Fischer, U. (2001). Methylation of Sm proteins by a complex containing PRMT5 and the putative U snRNP assembly factor pICln. Curr. Biol. 11, 1990-1994.

Mouaikel, J., Verheggen, C., Bertrand, E., Tazi, J., and Bordonné, R. (2002). Hypermethylation of the cap structure of both yeast snRNAs and snoRNAs requires a conserved methyltransferase that is localized to the nucleolus. Mol. Cell 9, 891-901.

Mouaikel, J., Narayanan, U., Verheggen, C., Matera, A.G., Bertrand, E., Tazi, J., and Bordonne, R. (2003). Interaction between the small-nuclear-RNA cap hypermethylase and the spinal muscular atrophy protein, survival of motor neuron. EMBO Rep. 4, 616-622.

Moy, T.I., and Silver, P.A. (2002). Requirements for the nuclear export of the small ribosomal subunit. J. Cell Sci. 115, 2985-2995.

Mroczek, S., Krwawicz, J., Kutner, J., Lazniewski, M., Kuciński, I., Ginalski, K., and Dziembowski, A. (2012). C16orf57, a gene mutated in poikiloderma with neutropenia, encodes a putative phosphodiesterase responsible for the U6 snRNA 3' end modification. 
Genes Dev. 26, 1911-1925.

Mullis, K., Faloona, F., Scharf, S., Saiki, R., Horn, G., and Erlich, H. (1986). Specific enzymatic amplification of DNA in vitro: The polymerase chain reaction. Cold Spring Harb. Symp. Quant. Biol. 51, 263-273.

Nesic, D., Tanackovic, G., and Kramer, A. (2004). A role for Cajal bodies in the final steps of U2 snRNP biogenesis. J. Cell Sci. 117, 4423-4433.

Noble, S.M., and Guthrie, C. (1996). Transcriptional pulse-chase analysis reveals a role for a novel snRNP-associated protein in the manufacture of spliceosomal snRNPs. EMBO J. 15, 4368-4379.

Ohno, M., Segref, A., Bachi, A., Wilm, M., and Mattaj, I.W. (2000). PHAX, a mediator of U snRNA nuclear export whose activity is regulated by phosphorylation. Cell 101, 187-198.

Olson, B.L., and Siliciano, P.G. (2003). A diverse set of nuclear RNAs transfer between nuclei of yeast heterokaryons. Yeast 20,893-903.

Palacios, I., Hetzer, M., Adam, S.A., and Mattaj, I.W. (1997). Nuclear import of U snRNPs requires importin beta. EMBO J. 16, 6783-6792.

Pemberton, L.F., Rosenblum, J.S., and Blobel, G. (1999). Nuclear import of the TATAbinding protein: Mediation by the karyopherin Kap114p and a possible mechanism for intranuclear targeting. J. Cell Biol. 145, 1407-1417.

Pettersson, I., Hinterberger, M., Mimori, T., Gottlieb, E., and Steitz, J.A. (1984). The structure of mammalian small nuclear ribonucleoproteins. J.Biol. Chem. 259, 5907-5914.

Pfaffl, M. (2004). Quantification strategies in real-time PCR Michael W . Pfaffl. A-Z Quant. PCR 87-112.

Plessel, G., Fischer, U., and Lührmann, R. (1994). m3G cap hypermethylation of U1 small nuclear ribonucleoprotein (snRNP) in vitro: evidence that the U1 small nuclear RNA(guanosine-N2)-methyltransferase is a non-snRNP cytoplasmic protein that requires a binding site on the Sm core domain. Mol. Cell. Biol. 14, 4160-4172.

Porrua, O., and Libri, D. (2015). Transcription termination and the control of the transcriptome: why, where and how to stop. Nat Rev Mol Cell Biol 16, 190-202.

Porrua, O., Hobor, F., Boulay, J., Kubicek, K., D’Aubenton-Carafa, Y., Gudipati, R.K., Stefl, 
R., and Libri, D. (2012). In vivo SELEX reveals novel sequence and structural determinants of Nrd1-Nab3-Sen1-dependent transcription termination. Embo J 31, 3935-3948.

Reddy, R., Henning, D., Das, G., Harless, M., and Wright, D. (1987). The capped U6 small nuclear RNA is transcribed by RNA polymerase III. J. Biol. Chem. 262, 75-81.

Reddy, R., Singh, R., and Shimba, S. (1992). Methylated cap structures in eukaryotic RNAs: Structure, synthesis and functions. Pharmacol. Ther. 54, 249-267.

Ribbeck, K., Lipowsky, G., Kent, H.M., Stewart, M., and Görlich, D. (1998). NTF2 mediates nuclear import of Ran. EMBO J. 17, 6587-6598.

Rose, M., Winston, F., and Hieter, P. (1991). Methods in Yeast Genetics - A Laboratory Course Manual. Biochem. Educ. 19, 101-102.

Roy, J., Zheng, B., Rymond, B.C., and Woolford, J.L. (1995). Structurally related but functionally distinct yeast Sm D core small nuclear ribonucleoprotein particle proteins. Mol. Cell. Biol. 15, 445-455.

Ryan, K.J., Zhou, Y., and Wente, S.R. (2007). The Karyopherin Kap95 Regulates Nuclear Pore Complex Assembly into Intact Nuclear Envelopes In Vivo. Mol. Biol. Cell 18, 886-898.

Rymond, B.C. (1993). Convergent transcripts of the yeast PRP38-SMD1 locus encode two essential splicing factors, including the D1 core polypeptide of small nuclear ribonucleoprotein particles. Proc. Natl. Acad. Sci. U. S. A. 90, 848-852.

Sambrook, J., Fritsch, E.F., and Maniatis, T. (1989). Molecular Cloning: A Laboratory Manual. Cold Spring Harbor laboratory press.

Sapra, A.K., Khandelia, P., and Vijayraghavan, U. (2008). The splicing factor Prp17 interacts with the U2, U5 and U6 snRNPs and associates with the spliceosome pre- and post-catalysis. Biochem. J. 416, 365-374.

Schramm, L., and Hernandez, N. (2002). Recruitment of RNA polymerase III to its target promoters. 2593-2620.

Schwer, B., and Shuman, S. (2015). Structure-function analysis and genetic interactions of the Yhc1, SmD3, SmB, and Snp1 subunits of yeast U1 snRNP and genetic interactions of SmD3 with U2 snRNP subunit Lea1. Rna 21, 1173-1186.

Schwer, B., Mao, X., and Shuman, S. (1998). Accelerated mRNA decay in conditional 
mutants of yeast mRNA capping enzyme. Nucleic Acids Res. 26, 2050-2057.

Schwer, B., Erdjument-Bromage, H., and Shuman, S. (2011). Composition of yeast snRNPs and snoRNPs in the absence of trimethylguanosine caps reveals nuclear cap binding protein as a gained U1 component implicated in the cold-sensitivity of tgs1?? cells. Nucleic Acids Res. $39,6715-6728$.

Schwer, B., Kruchten, J., and Shuman, S. (2016). Structure-function analysis and genetic interactions of the SmG, SmE, and SmF subunits of the yeast Sm protein ring. RNA 22, $1320-1328$.

Schwer, B., Roth, A.J., and Shuman, S. (2017). Will the circle be unbroken: specific mutations in the yeast Sm protein ring expose a requirement for assembly factor Brr1, a homolog of Gemin2. Rna rna.059881.116.

Seedorf, M., and Silver, P.A. (1997). Importin/karyopherin protein family members required for mRNA export from the nucleus. Proc. Natl. Acad. Sci. U. S. A. 94, 8590-8595.

Segref, A., Sharma, K., Doye, V., Hellwig, A., Huber, J., Lührmann, R., and Hurt, E. (1997). Mex67p, a novel factor for nuclear mRNA export. Binds to both poly(A)+ RNA and nuclear pores. EMBO J. 16, 3256-3271.

Seipelt, R.L., Zheng, B., Asuru, A., and Rymond, B.C. (1999). U1 snRNA is cleaved by RNase III and processed through an Sm site-dependent pathway. Nucleic Acids Res. 27, 587595.

Senger, B., Simos, G., Bischoff, F.R., Podtelejnikov, A., Mann, M., and Hurt, E. (1998). Mtr10p functions as a nuclear import receptor for the mRNA-binding protein Np13p. EMBO J. 17, 2196-2207.

Séraphin, B. (1995). Sm and Sm-like proteins belong to a large family: identification of proteins of the U6 as well as the U1, U2, U4 and U5 snRNPs. EMBO J. 14, 2089-2098.

Shen, E.C., Stage-Zimmermann, T., Chui, P., and Silver, P.A. (2000). 7The yeast mRNAbinding protein Np13p interacts with the cap-binding complex. J. Biol. Chem. 275, 2371823724.

Sherman, F. (2002). Getting started with yeast. Methods Enzymol. 350, 3-41.

Shimba, S., and Reddy, R. (1994). Purification of human U6 small nuclear RNA capping enzyme. Evidence for a common capping enzyme for gamma-monomethyl-capped small 
RNAs. J. Biol. Chem. 269, 12419-12423.

Shukla, S., and Parker, R. (2014). Quality control of assembly-defective U1 snRNAs by decapping and 5'-to-3' exonucleolytic digestion. TL - 111. Proc. Natl. Acad. Sci. U. S. A. $111 \mathrm{VN}-, 86$.

Sikorski, R.S., and Hieter, P. (1989). A system of shuttle vectors and yeast host strains designed for efficient manipulation of DNA in Saccharomyces cerevisiae. Genetics 122, 1927.

Siliciano, P.G., Kivens, W.J., and Guthrie, C. (1991). More than half of yeast U1 snRNA is dispensabel for growth. Nucleic Acids Res. 19, 6367-6372.

Singh, R., and Reddy, R. (1989). Gamma-monomethyl phosphate: a cap structure in spliceosomal U6 small nuclear RNA. Proc. Natl. Acad. Sci. U. S. A. 86, 8280-8283.

Sklenar, A.R., and Parthun, M.R. (2004). Characterization of yeast histone H3-specific type B histone acetyltransferases identifies an ADA2-independent Gcn5p activity. BMC Biochem. 5, $1-12$.

Sleeman, J. (2013). Small nuclear RNAs and mRNAs: linking RNA processing and transport to spinal muscular atrophy. Biochem. Soc. Trans. 41, 871-875.

Sloan, K.E., Gleizes, P.E., and Bohnsack, M.T. (2016). Nucleocytoplasmic Transport of RNAs and RNA-Protein Complexes. J. Mol. Biol. 428, 2040-2059.

Snay-Hodge, C.A., Colot, H. V., Goldstein, A.L., and Cole, C.N. (1998). Dbp5p/Rat8p is a yeast nuclear pore-associated DEAD-box protein essential for RNA export. EMBO J. 17, 2663-2676.

Solsbacher, J., Maurer, P., Bischoff, F.R., and Schlenstedt, G. (1998). Cse1p is involved in export of yeast importin alpha from the nucleus. Mol. Cell. Biol. 18, 6805-6815.

Spiller, M.P., Boon, K.L., Reijns, M.A.M., and Beggs, J.D. (2007). The Lsm2-8 complex determines nuclear localization of the spliceosomal U6 snRNA. Nucleic Acids Res. 35, 923929.

Sprague, G.F. (1991). Assay of yeast mating reaction. Methods Enzymol. 194, 77-93.

Steinmetz, E.J., Conrad, N.K., Brow, D.A., and Corden, J.L. (2001). RNA-binding protein Nrd1 directs poly(A)-independent 3'-end formation of RNA polymerase II transcripts. Nature 
$413,327-331$.

Sträßer, K., Baßler, J., and Hurt, E. (2000). Binding of the Mex67p/Mtr2p heterodimer to FXFG, GLFG, and FG repeat nucleoporins is essential for nuclear mRNA export. J. Cell Biol. $150,695-706$.

Ström, A.C., and Weis, K. (2001). Importin-beta-like nuclear transport receptors. Genome Biol. 2, REVIEWS3008.

Taura, T., Krebber, H., and Silver, P. a (1998). A member of the Ran-binding protein family, Yrb2p, is involved in nuclear protein export. Proc. Natl. Acad. Sci. U. S. A. 95, 7427-7432.

Tieg, B., and Krebber, H. (2013). Dbp5 - From nuclear export to translation. Biochim. Biophys. Acta - Gene Regul. Mech. 1829, 791-798.

Titov, A.A., and Blobel, G. (1999). The karyopherin Kap122p/Pdr6p imports both subunits of the transcription factor IIA into the nucleus. J. Cell Biol. 147, 235-245.

Towbin, H., Staehelin, T., and Gordon, J. (1979). Electrophoretic transfer of proteins from polyacrylamide gels to nitrocellulose sheets: procedure and some applications. Proc. Natl. Acad. Sci. 76, 4350-4354.

Turowski, T.W., and Tollervey, D. (2016). Transcription by RNA polymerase III: insights into mechanism and regulation. Biochem. Soc. Trans. 44, 1367-1375.

Vasianovich, Y., and Wellinger, R.J. (2017). Life and Death of Yeast Telomerase RNA. J. Mol. Biol.

Will, C.L., and Lührmann, R. (2001). Spliceosomal UsnRNP biogenesis, structure and function. Curr. Opin. Cell Biol. 13, 290-301.

Wilusz, C.J., and Wilusz, J. (2013). Lsm proteins and Hfq: Life at the 3' end. RNA Biol. 10, 592-601.

Windgassen, M., Sturm, D., Cajigas, I.J., Gonzalez, C.I., Seedorf, M., Bastians, H., and Krebber, H. (2004). Yeast Shuttling SR Proteins Npl3p, Gbp2p, and Hrb1p Are Part of the Translating mRNPs, and Npl3p Can Function as a Translational Repressor. Mol. Cell. Biol. 24, 10479-10491.

Winston, F., Dollard, C., and Ricupero-Hovasse, S.L. (1995). Construction of a set of convenient Saccharomyces cerevisiae strains that are isogenic to S288C. Yeast 11, 53-55. 
Wu, H., Becker, D., and Krebber, H. (2014). Telomerase RNA TLC1 shuttling to the cytoplasm requires mRNA export factors and is important for telomere maintenance. Cell Rep. 8, 1630-1638.

Xiao, Z., McGrew, J.T., Schroeder, a J., and Fitzgerald-Hayes, M. (1993). CSE1 and CSE2, two new genes required for accurate mitotic chromosome segregation in Saccharomyces cerevisiae. Mol. Cell. Biol. 13, 4691-4702.

Yao, W., Roser, D., Köhler, A., Bradatsch, B., Baßler, J., and Hurt, E. (2007). Nuclear Export of Ribosomal 60S Subunits by the General mRNA Export Receptor Mex67-Mtr2. Mol. Cell $26,51-62$.

Zander, G., Hackmann, A., Bender, L., Becker, D., Lingner, T., Salinas, G., and Krebber, H. (2016). mRNA quality control is bypassed for immediate export of stress-responsive transcripts. Nature.

Zhang, R., So, B.R., Li, P., Yong, J., Glisovic, T., Wan, L., and Dreyfuss, G. (2011). Structure of a key intermediate of the SMN complex reveals Gemin2's crucial function in snRNP assembly. Cell 146, 384-395.

Zhang, Z., Lotti, F., Dittmar, K., Younis, I., Wan, L., Kasim, M., and Dreyfuss, G. (2008). SMN Deficiency Causes Tissue-Specific Perturbations in the Repertoire of snRNAs and Widespread Defects in Splicing. Cell 133, 585-600. 


\section{Acknowledgement - Danksagung}

An dieser Stelle möchte ich mich bei allen bedanken, die mich bei der Anfertigung dieser Arbeit unterstützt haben.

Mein Dank gilt in besonderer Weise Prof. Dr. Heike Krebber, die mir die Anfertigung meiner Dissertation und die Arbeit an weiteren interessanten Themen in ihrer Arbeitsgruppe ermöglicht hat. Vielen Dank für die enge Betreuung und die hervorragende wissenschaftliche Förderung.

Ebenfalls möchte ich den Mitgliedern meines Thesis committees, Prof. Dr. Ralf Ficner und Prof. Dr. Ralph Kehlenbach, für ihre wissenschaftliche Begleitung danken. Herzlich danken möchte ich auch PD Dr. Wilfried Kramer und Dr. Achim Dickmanns, die mir während meiner Arbeit immer mit Rat und Tat zur Seite gestanden haben.

Des Weiteren möchte ich mich bei allen aktuellen und ehemaligen Mitgliedern der AG Krebber und AG Bastians für ihre Hilfsbereitschaft, die angenehme Arbeitsatmosphäre, den starken Zusammenhalt und die hilfreichen Diskussionen bedanken. Mein besonderer Dank gilt Dr. Lysann Bender, Dr. Bettina Neumann, Lena Söldner, Dr. Haijia Wu und Dr. Gesa Zander.

Abschließend gilt mein herzlicher Dank meiner Familie und besonders meiner Frau Laura und meinem Sohn Philipp. Ihr habt mich während der letzten Jahre immer unterstützt, motiviert und an mich geglaubt. 